\title{
ANALYSIS OF VOICE PERTURBATIONS USING AN ASYMMETRIC MODEL OF THE VOCAL FOLDS
}

\author{
Marco Nardone
}

\begin{abstract}
A Thesis
Submitted to the Graduate College of Bowling Green State University in partial fulfillment of the requirements for the degree of
\end{abstract}

\section{MASTER OF SCIENCE}

August 2007

Committee:

Lewis P. Fulcher, Advisor

Robert I. Boughton

Ronald C. Scherer 


\begin{abstract}
\end{abstract}
Lewis P. Fulcher, Advisor

A mathematical model was developed to investigate possible causes of jitter and shimmer. The model builds from the classic, lumped element, two-mass model of Ishizaka and Flanagan. Bilateral asymmetry was incorporated into the model to facilitate the simulation of physiological and aerodynamic imbalances in the larynx. The aerodynamic driving forces on the vocal folds were derived from empirical pressure data that were obtained from a scaled-up Plexiglas model of a typical male human larynx, called M5. The mathematical model is based on ten, second-order, nonlinear, coupled, ordinary differential equations that were solved simultaneously using the software Mathematica. The solutions were analyzed graphically and numerically to identify perturbations in the fundamental frequency and amplitude of the glottal airflow. Jitter and shimmer were quantified using the jitter factor and the amplitude variability index.

The model was employed to study the effects of time-dependent, randomly varying, asymmetries in the stiffness of the vocal folds, and the driving forces for the case of an excised larynx. Time-independent asymmetries in the mass, stiffness, and driving forces were also examined. The results indicate that only time-dependent asymmetries result in jitter and shimmer. The magnitudes of jitter and shimmer are less than those observed in the natural sounding voice, even when the asymmetries are large. One of the most interesting results of our study is that although time-independent asymmetries cause the right and left vocal folds to 
oscillate out of phase or with different amplitudes, the vocal folds entrain and vibrate at a common frequency. 
To Isabella Kathryn,

Thank you for being born. 


\section{ACKNOWLEDGMENTS}

I gratefully acknowledge the support that I received from the Department of Physics and Astronomy at Bowling Green State University for twice awarding me the J. Robert and Gretchen Overman Scholarship Award. I would also like to thank the Graduate College and Bookstore for providing me with support from the Katzner Funds for Graduate Student Research and Professional Development.

I would like to thank my committee, especially Dr. Lewis Fulcher, my advisor. Not only did Dr. Fulcher teach me the fundamental physics essential to this task, he also instilled in me a great appreciation for the theoretical physicist's quest to better understand Mother Nature. For this, I am truly grateful. I also thank him for selflessly sharing his wisdom and knowledge over the last two years. I would also like to thank Dr. Ronald Scherer for his insights, advice, and humor that always made our meetings something to look forward to. I extend my gratitude to Dr. Robert Boughton as well for his patience and commitment to teaching.

My deepest appreciation goes to my wife, Shannon Orr, for her encouragement, support and advice. I am forever grateful for her encouragement and enthusiasm as I work towards obtaining my Ph.D. in Physics. I am truly fortunate to have such a supportive partner. I would also like to thank my newborn daughter Isabella for her smiles and coos that made it a pleasure to write a thesis without any sleep. 


\section{TABLE OF CONTENTS}

Page

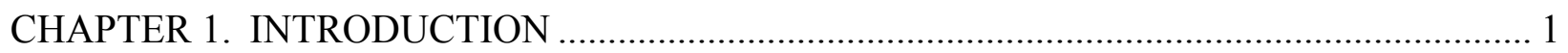

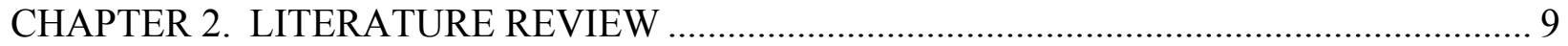

2.1 Overview of Vocal System Models ............................................................ 9

2.1.1 The Two-Mass Model of Ishizaka and Flanagan (1972) ........................ 11

2.1.2 The Asymmetric Two-Mass Model of Isshiki and Ishizaka (1976)......... 20

2.1.3 The 16-mass Model of Titze (1973)................................................ 21

2.1.4 The Wave-Reflection Model of the Vocal Tract.................................... 22

2.1.5 The Body-Cover Model of Story and Titze (1995) ............................. 23

2.2 Overview of Voice Perturbation Measures ...................................................... 27

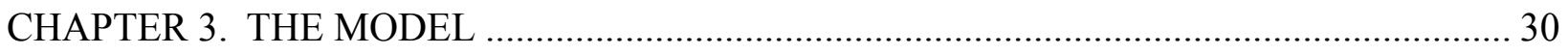

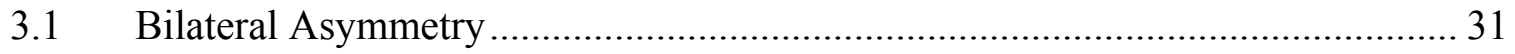

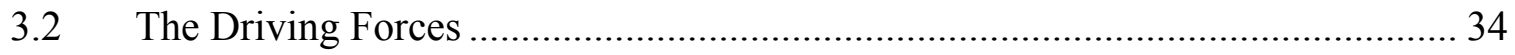

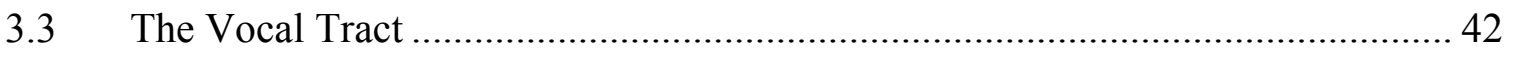

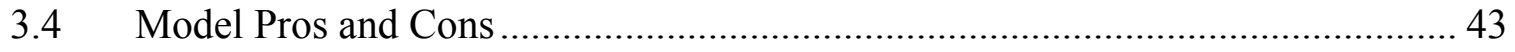

CHAPTER 4. METHODOLOGY ................................................................................ 46

4.1 Testing Hypothesis \#1: Time-independent Physical Asymmetry ...................... 49

4.2 Testing Hypothesis \#2: Time-dependent Physical Asymmetry ......................... 50

4.3 Testing Hypothesis \#3: Time-independent Asymmetric Driving Forces ............. 52

4.4 Testing Hypothesis \#4: Time-dependent Asymmetric Driving Forces ............... 52

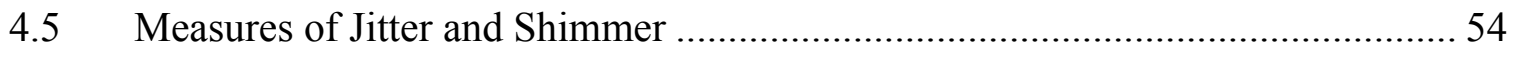

CHAPTER 5. RESULTS AND DISCUSSION ............................................................. 56 
5.1 Hypothesis \#1: Time-independent Physical Asymmetry .................................. 57

5.1.1 Asymmetry in the Lower, Right Mass ............................................... 57

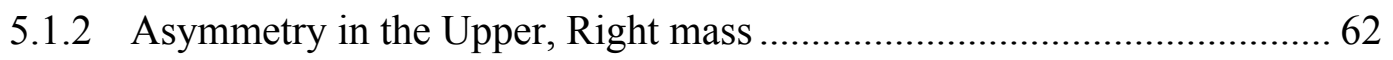

5.1.3 Asymmetry in the Lower, Right Spring Force.................................... 65

5.1.4 Asymmetry in the Upper, Right Spring Force ................................. 65

5.1.5 Asymmetry in the Right, Coupling Spring Force ............................... 69

5.2 Hypothesis \#2: Time-dependent Physical Asymmetry.................................. 73

5.2.1 Random Asymmetry in the Lower, Right Spring Constant ................... 73

5.2.2 Random Asymmetry in the Lower, Right and Left Spring Constants ...... 78

5.2.3 Random Asymmetry in All Spring Constants .................................. 81

5.3 Hypothesis \#3: Time-independent Asymmetry in the Driving Forces ................ 84

5.4 Hypothesis \#4: Time-dependent Asymmetry in the Driving Forces ................... 87

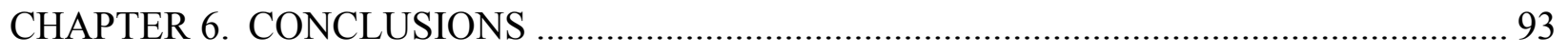

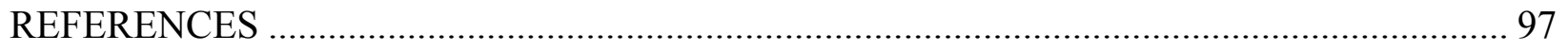




\section{LIST OF FIGURES}

Page

Figure 1-1 Schematic of the vocal airways (source: Flanagan 1972)............................... 1

Figure 1-2 Top view of the vocal folds (a) during normal breath, and (b) closed prior to phonation (Voice Medicine 2006).

Figure 1-3 Structure of the vocal folds (a) at rest (b) experiencing wave motion (Hanavan 2006). 3

Figure 1-4 Phases of the vocal fold oscillation cycle as described in the running text (source: Hanavan, 2006)

Figure 2-1 Schematic of the IF72 model of the vocal folds: side view (based on: Ishizaka and Flanagan 1972)......

Figure 2-2 Schematic of the IF72 model vocal folds: top view (based on: Ishizaka and Flanagan 1972).

Figure 2-3 IF72 network representation of the glottis and vocal tract (source: Ishizaka and Flanagan 1972)

Figure 2-4 Schematic of the three-mass body-cover model (based on: Story and Titze 1995)

Figure 2-5 Muscle control parameters for (a) the IF72 model and (b) the body-cover model...

Figure 3-1 Schematic of the asymmetric two-mass model............................................. 32

Figure 3-2 Schematic of the M5 apparatus (source: Scherer and Shinwari 2001) ............... 35

Figure 3-3 Schematic of vocal fold geometry (source: Scherer et al. 2001) ........................ 35

Figure 3-4 Sample of M5 experimental data for a converging angle of $-20^{0}$ (source: Scherer)

Figure 3-5 Sample of M5 interpolation function data in graphical form (Mathematica output). The glottal entrance is located at $\mathrm{z}=0.2114 \mathrm{~cm}$, and the glottal exit is

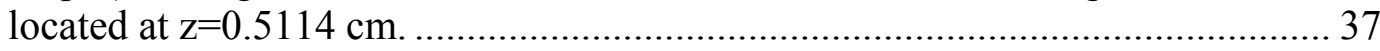

Figure 3-6 Identification of M5 variables in the asymmetric two-mass model................... 39

Figure 3-7 Driving force on mass $m_{R 1}$ for the typical IF72 model (upper), the modified IF72 force (center), and the M5 experimental data (lower). The transglottal pressure is $8 \mathrm{~cm} \mathrm{H}_{2} \mathrm{O}$ and the glottal area is $0.05 \mathrm{~cm}^{2}$. 
Figure 5-1 Results for the typical parameter set as given in Table 4-1 and applying the M5 pressure data to calculate the driving forces: (a) oscillator displacement; (b) glottal airflow rate; and (c) output sound pressure level. 56

Figure 5-2 Fundamental period contour normalized to the average period and glottal airflow amplitude contour normalized to the average amplitude for the control case. ..... 58

Figure 5 -3 Results for an increase in the lower, right mass by a factor of 1.50. (a) oscillator displacements, (b) glottal flow, and (c) output sound pressure level................... 59

Figure 5-4 Results for an increase in the lower, right mass by a factor of 2.00: (a) oscillator displacements, (b) glottal flow, and (c) output sound pressure level. 60

Figure 5-5 Fundamental period contour normalized to the average period and glottal airflow amplitude contour normalized to the average amplitude when $m_{R 1}$ is increased by a factor of 2.00 . 61

Figure 5-6 Fundamental frequency and phase difference between the right and left vocal

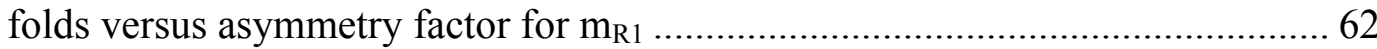

Figure 5-7 Results for an increase in the upper, right mass by a factor of 2.00: (a) oscillator displacements, (b) glottal flow, and (c) output sound pressure level.

Figure 5-8 Fundamental period contour normalized to the average period and glottal airflow amplitude contour normalized to the average amplitude when $\mathrm{m}_{\mathrm{R} 2}$ is increased by a factor of 2.00 . 64

Figure 5-9 Displacement diagrams for increasing $k_{R 1}$ by factors of $1.05,1.10,1.20,1.50$, and 2.00 (from top to bottom). 66

Figure 5-10 Fundamental period contour normalized to the average period and glottal airflow amplitude contour normalized to the average amplitude when $k_{R 1}$ is increased by a factor of 2.00 . 67

Figure 5-11 Fundamental period contour normalized to the average period and glottal airflow amplitude contour normalized to the average amplitude when $\mathrm{k}_{\mathrm{R} 2}$ is increased by a

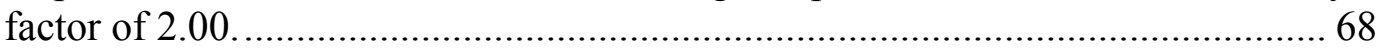

Figure 5-12 Displacement diagrams for increasing $\mathrm{k}_{\mathrm{R} 2}$ by factors of $1.05,1.10,1.20,1.50$, and 2.00 (from top to bottom). 70

Figure 5-13 Fundamental period contour normalized to the average period and glottal airflow amplitude contour normalized to the average amplitude for the case of $\mathrm{k}_{\mathrm{Rc}}$ increased by a factor of 2.00 71

Figure 5-14 Displacement diagrams for increasing $\mathrm{k}_{\mathrm{Rc}}$ by factors of $1.05,1.10,1.20,1.50$, and 2.00 (from top to bottom). 72 
Figure 5-15 Time-dependence of $k_{\mathrm{R} 1}$ for $1 \%$ (top) and $25 \%$ (bottom) random variations....... 74

Figure 5-16 Results for random fluctuations in the lower, right spring force $\mathrm{k}_{\mathrm{R} 1}$ by $25 \%$, (a) oscillator displacements, (b) glottal flow, and (c) output sound pressure level.... 75

Figure 5-17 Fundamental period contour normalized to the average period and glottal airflow amplitude contour normalized to the average amplitude for random fluctuations of

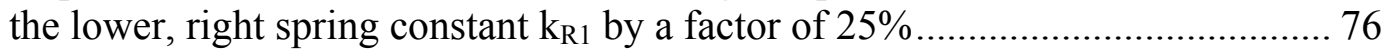

Figure 5-18 Jitter Factor and Amplitude Variability Index versus the magnitude of random fluctuations of $\mathrm{k}_{\mathrm{R} 1}$. 77

Figure 5-19 Results for random fluctuations in the lower, right and left spring forces, $\mathrm{k}_{\mathrm{R} 1}$ and $\mathrm{k}_{\mathrm{L} 1}$, by $25 \%$, (a) oscillator displacements, (b) glottal flow, and (c) output sound pressure level.

Figure 5-20 Fundamental period contour normalized to the average period and glottal airflow amplitude contour normalized to the average amplitude for random fluctuations of the lower, right spring constants $\mathrm{k}_{\mathrm{R} 1}$ and $\mathrm{k}_{\mathrm{L} 1}$ by a factor of $25 \%$ 79

Figure 5-21 Jitter Factor and Amplitude Variability Index versus the magnitude of random fluctuations of $\mathrm{k}_{\mathrm{R} 1}$ and $\mathrm{k}_{\mathrm{L} 1}$.

Figure 5-22 Results for random fluctuations in all of the spring forces by $25 \%$, (a) oscillator displacements, (b) glottal flow, and (c) output sound pressure level.................. 81

Figure 5-23 Fundamental period contour normalized to the average period and glottal airflow amplitude contour normalized to the average amplitude for random fluctuations of all spring constants by a factor of $25 \%$.

Figure 5-24 Jitter Factor and Amplitude Variability Index versus the magnitude of random fluctuation of all spring constants.

Figure 5-25 Driving forces on the lower masses during phonation. 85

Figure 5-26 Oscillator displacement graphs for increased lower, right driving force $F_{R 1}$ by factors of $1.05,1.10,1.20,1.50$, and 2.00 (from top to bottom).. 86

Figure 5-27 Fundamental period contour normalized to the average period and glottal airflow amplitude contour normalized to the average amplitude for $+/-50 \%$ random fluctuations of the driving force on the lower, right mass during the divergent part of the cycle. 88

Figure 5-28 Fundamental period contour normalized to the average period and glottal airflow amplitude contour normalized to the average amplitude for $+/-50 \%$ random fluctuations of both driving forces on the right vocal fold. 89 
Figure 5-29 Jitter Factors and Amplitude Variability Index for random fluctuations of the

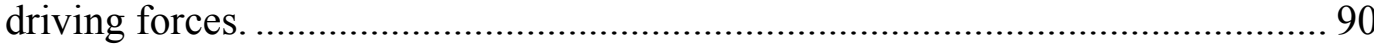

Figure 5-30 Fundamental period contour normalized to the average period and glottal airflow amplitude contour normalized to the average amplitude when the fluctuating forces on the right vocal fold are increase by as much as a factor of two. 


\section{LIST OF TABLES}

Page

Table 2-1 Force conditions for the IF72 model .......................................................... 18

Table 2-2 Comparison of biomechanical parameters in the IF72 model and the body-cover

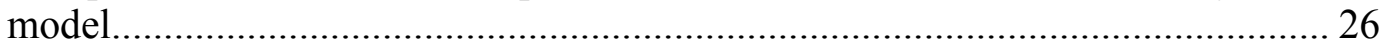

Table 3-1 Force conditions if the modified IF72 model pressures are applied .................. 42

Table 3-2 Force conditions if the M5 pressure data is applied ...................................... 42

Table 4-1 List of parameters and their values for the asymmetric two-mass model............ 48

Table 4-2 Parameter modifications for testing hypothesis \#1 ......................................... 49

Table 4-3 Parameter modifications for testing hypothesis \#2 ...................................... 50

Table 4-4 Parameter modifications for testing hypothesis \#3 ....................................... 52

Table 4-5 Parameter modifications for testing hypothesis \#4 ...................................... 53 


\section{CHAPTER 1. INTRODUCTION}

Voiced sounds begin with a conscious increase in air pressure in the lungs. This elevated pressure allows for the control of airflow through the vocal system, including the trachea, larynx, vocal tract, oral cavity and nasal cavity. A schematic of the vocal airways is presented in Figure1-1.

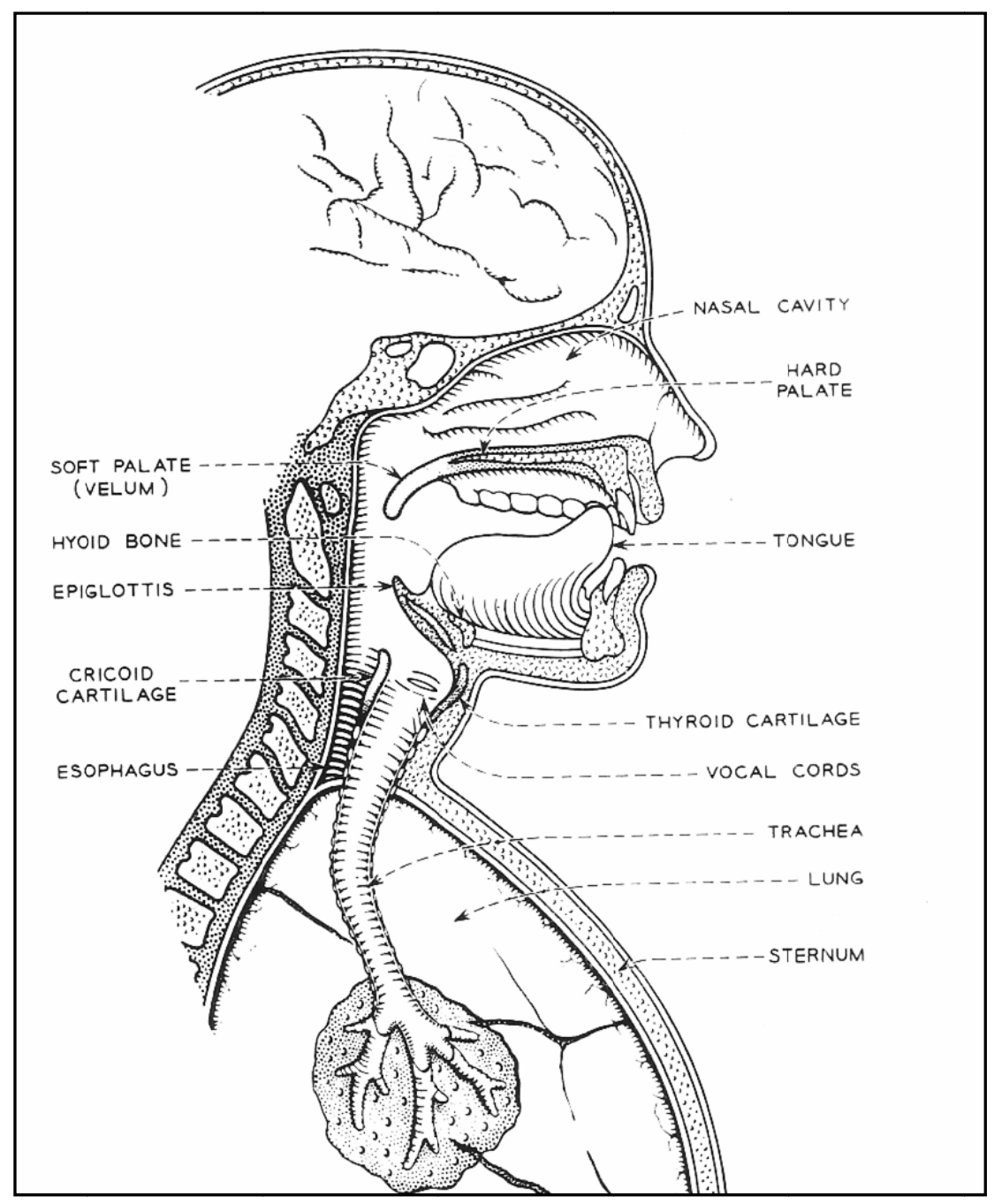

Figure 1-1 Schematic of the vocal airways (source: Flanagan 1972)

Sound is essentially a fluctuation in the density of the medium which carries the disturbance. In the case of voiced sounds, this fluctuation of the medium is caused by changing 
airflow above the vocal folds associated with oscillation of the vocal folds, which are located in the larynx. The act of producing voiced sounds in this manner is called phonation. Prior to phonation the vocal folds are brought into close proximity to each other by muscular control of the speaker. As air passes through the narrow slit (glottis) between the vocal folds, energy is transferred from the airflow to the folds, resulting in oscillation. If the pressure in the glottis is sufficient to overcome the inertial and damping forces of the vocal folds, then a limit cycle may be achieved, whereby the input energy from the flowing air equals the energy losses, and the vibration is sustained over a period of time. The oscillation of the folds results in a valving of the otherwise continuous airflow into pulses. These pulses are then modified by the downstream vocal (especially vocal tract inertance) to produce the vocal source sound. The configurations of the vocal folds during regular breathing and just prior to phonation are presented in Figure 1.2.

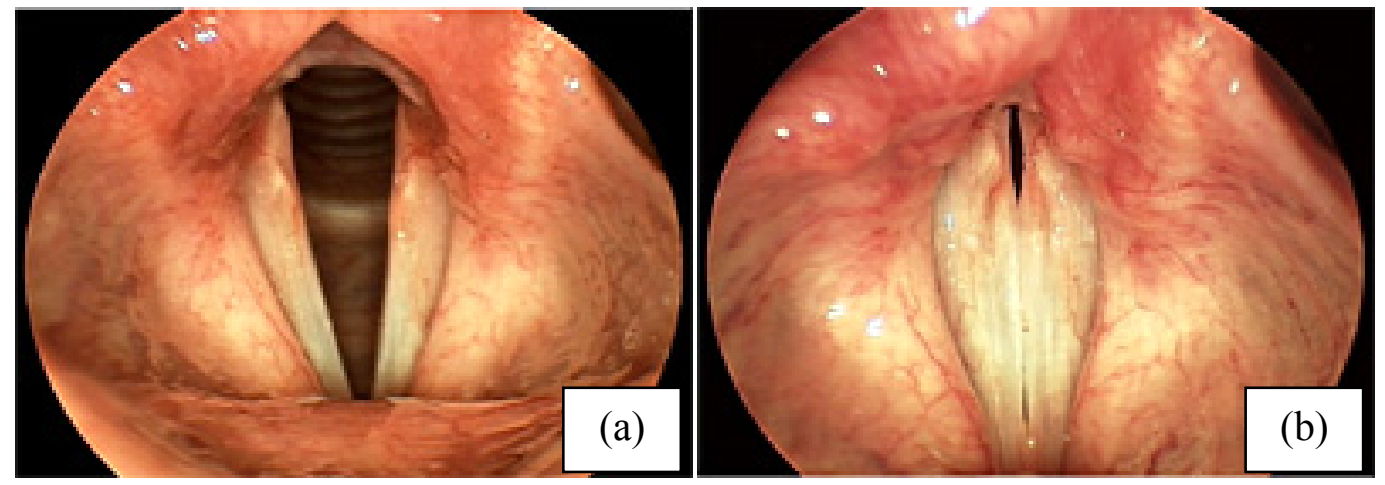

Figure 1-2 Top view of the vocal folds (a) during normal breath, and (b) closed prior to phonation (Voice Medicine 2006).

The outer layers of the vocal folds consist of a $50 \mu \mathrm{m}$-thick layer of stratified epithelium and a $1 \mathrm{~mm}$ to $2 \mathrm{~mm}$ thick lamina propria (Hirano 1975). The epithelium and the outer (superficial) layer of the lamina propria are collectively called the mucosa. Vocal fold vibration is largely dependent upon a surface wave that is excited in the mucosa, referred to as a mucosal wave, by the airflow through the glottis (the open space between the vocal folds). The mucosa consists of a network of elastin and collagen fibers within an interstitial fluid composed of 
proteoglycans and structured glycoproteins. The fibers provide the elastic properties of the mucosa while the interstitial fluid is responsible for the viscous properties (Titze, Schmidt and Titze 1995). The vocal folds are also comprised of muscle fiber which support and control the vocal folds. The structures of the vocal folds at rest and during a mucosal wave are presented in Figure 1-3.

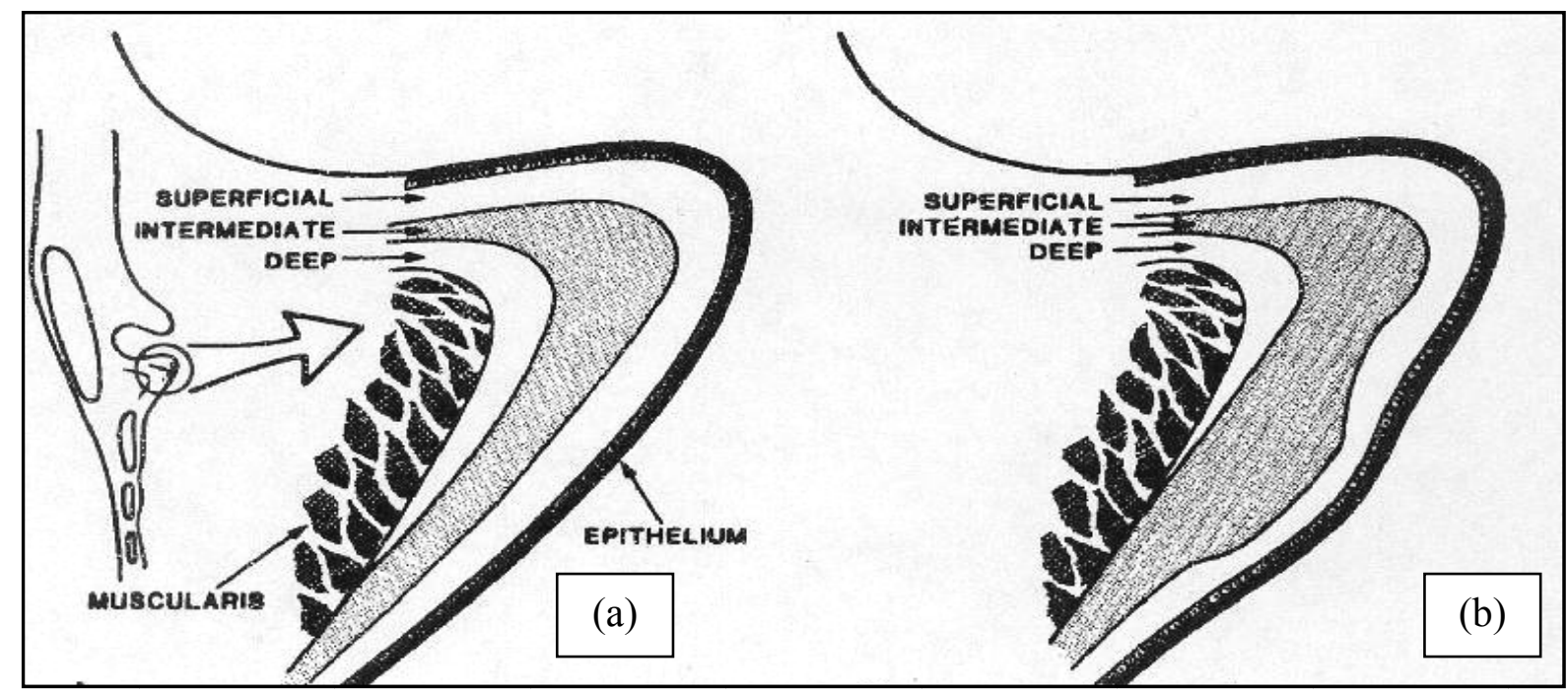

Figure 1-3 Structure of the vocal folds (a) at rest (b) experiencing wave motion (Hanavan 2006).

The elegant interplay between the aerodynamic forces and the oscillating vocal folds is simple to describe verbally, yet extremely complex to model mathematically. Succinctly speaking, the aerodynamic forces drive the geometrical shapes of the vocal folds which, in turn, modify the forces. While this feedback loop (or coupling) is continuous in the time domain, the main stages are presented in the frames of Figure 1-4.

As shown in the eight frames of Figure 1-4, the pressure beneath the closed vocal folds begins to separate the lower portions of the glottal walls (frame 2). As the vocal folds separate, a "convergent" shape is produced (frame 4), and the folds continue to move outward. The mucosal wave that is generated on the pliable medial surface of the folds has a wave speed on 

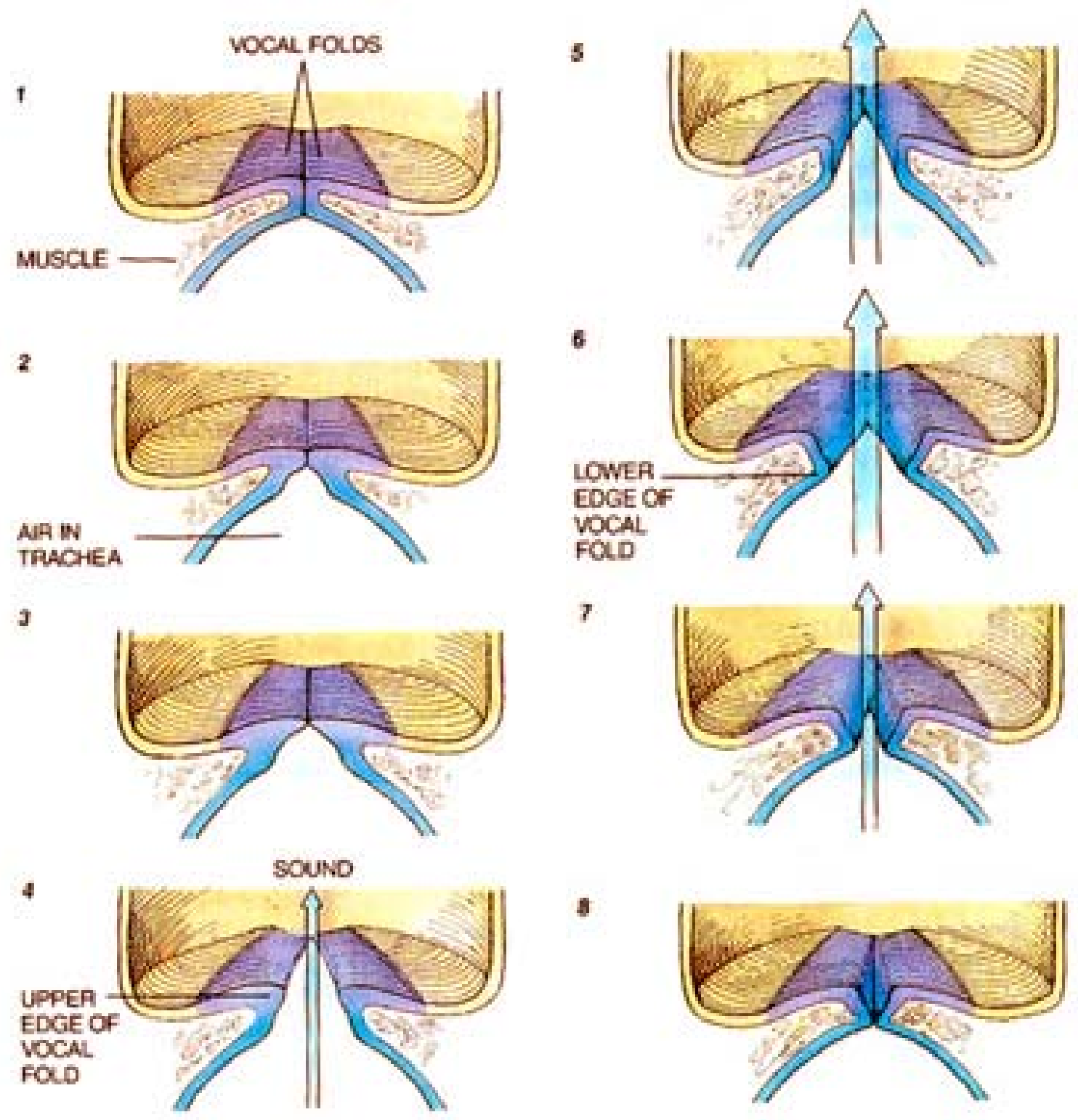

Figure 1-4 Phases of the vocal fold oscillation cycle as described in the running text (source: Hanavan, 2006) 
the order of $100 \mathrm{~cm} / \mathrm{s}$ (Baer 1975). The wave is often described as a vertical phase difference between the lower and upper glottal surfaces of the vocal folds. When the maximum excursion from the midline is achieved, the lower portion of the folds turn back towards the midline while the top portions continue with the outward motion (frame 5). By the time the top portions of the folds begin the return journey to the centerline the vocal folds have achieved a "divergent" glottal shape (frame 6). This new configuration modifies the aerodynamics such that a partial vacuum arises between the folds, which causes an inward air pressure force to be applied to the vocal folds, thereby encouraging the return to the centerline position. At the centerline, the folds collide, and the cycle is repeated (frames 7 and 8 ). While this process is occurring, several complexities in the fluid mechanics arise such as turbulence, generation of vortices, and airflow jet formation. These complexities lead to uncertain and unpredictable conditions in the intraglottal pressures. As will be discussed in detail later, one of the goals of this study is to investigate the possible effects of these uncertainties on the periodicity of voiced sound, without having a complete knowledge of the complex fluid mechanical details.

In addition to vocal fold modeling, an understanding of what happens to the airflow and pressure between the glottis and the external environment is required. Typically, this is considered a source/filter problem where the sound source is created in the glottis, and the vocal tract is the resonant filter. The resonant characteristics of the vocal tract are determined by the shape selected by the speaker. The complex geometry and unsteady airflow through the resonant filter is another complex modeling task which is further complicated by the coupling between the source and the filter. Due to the many layers of complexity, several imaginative (but well-substantiated) simplifications are required to model the vocal system. 
As one may expect in a natural system, the properties of the left and right vocal folds may not be completely identical. In addition, the aeroacoustic and biomechanical parameters may vary from cycle to cycle during phonation. Therefore, both spatial and temporal asymmetries should be considered. These deviations in symmetry are in part responsible for the naturalness and richness of the human voice. However, an unnatural or hoarse sounding voice can result if the asymmetries become too large, due to pathological conditions or voice disorders. Although there are many ways to measure irregularities in voiced sounds, the two indicators in widespread use are jitter, or perturbations in the fundamental frequency of voiced sound, and shimmer, or perturbations in the amplitude of voiced sound.

It is generally accepted that jitter and shimmer are greater in pathological voices than in normal voices and that these irregularities abate when corrective action is successful. However, relationships between the magnitudes of jitter and shimmer to the specific abnormality of glottal function are poorly understood (Baken and Orlikoff 2000). This study aims to explore the connection of jitter and shimmer to specific causes. A deeper understanding of the connection between voice perturbation measures and the underlying causes should lead to improved understanding, diagnoses, and treatment of voice disorders.

The primary research question of this study is: What are the possible causes of jitter and shimmer in the human voice? Consequently, the objectives are:

1) to develop a model of the human voice suitable for the study of voice perturbations;

2) to investigate possible causes of these perturbations.

The hypotheses of this study are that jitter and/or shimmer are caused by:

1) time-independent asymmetries in the physical characteristics of the left and right vocal folds; 
2) time-dependent asymmetries in the physical characteristics of the left and right vocal folds;

3) time-independent asymmetries in the driving forces on the left and right vocal folds;

4) time-dependent asymmetries in the driving forces on the left and right vocal folds.

To test these hypotheses, a mathematical model was developed to facilitate the systematic modification of aerodynamic and biomechanical parameters, in order to determine the effects of these modifications on the periodicity and amplitude of the output sound wave. The basic approach to the model was to minimize the number of degrees of freedom while allowing for sufficient generality so that measurable signatures of the perturbations could be detected. The model is essentially a set of nonlinear, coupled differential equations describing the motion of the vocal folds, the aerodynamic forces that drive them, and the subsequent filtering of the pressure wave as it passes through the vocal tract and emanates from the lips. By solving these equations simultaneously, given appropriate biomechanical parameters and initial conditions, the subsequent motion of the vocal folds, the airflow rates, and the characteristics of the voiced sounds can be calculated. The system of equations is solved numerically using the software Mathematica. The results are also analyzed graphically and numerically using this software.

The aerodynamic forces employed in this model were obtained in part from the experimental results of a scaled-up Plexiglas model of the male vocal folds (model M5) located at the Department of Communication Disorders at Bowling Green State University (BGSU). The M5 experiments resulted in detailed measurements of the pressure distributions along the medial surfaces of vocal folds for various geometrical configurations and subglottal pressures.

Although a great deal of research has been done on such models, very little has been done to investigate the effects that asymmetries in the physical parameters and the driving forces have 
on the dynamics of the vocal system. A knowledge gap exists in the literature with respect to understanding the aerodynamic forces that drive the oscillating vocal folds. In an attempt to work towards closing that gap, the model used in this study converts the static results of the M5 model into dynamic driving forces that depend on the geometry of the vocal folds and the subglottal pressure. Although there are limitations to this approach, it provides some level of correction to typical theoretical models used in the past, and it may provide some guidance for future theoretical studies. The mathematical model employed here can be refined by incorporating additional empirical data, as it becomes available. 


\section{CHAPTER 2. LITERATURE REVIEW}

\subsection{Overview of Vocal System Models}

Mathematical models of the human voice have been developed for various reasons since the advent of computer technology. The two primary reasons have been: (1) to synthesize the human voice for the purposes of electronic reproduction; and (2) to acquire a greater understanding of voice pathologies and possible treatments for voice disorders. This study deals with the latter reason and, therefore, the model must have parameters that can be varied to simulate different conditions produced by various laryngeal adjustments or by pathological variations (Hirano 1974).

Vocal fold models have been developed with a broad spectrum of complexity depending on the research question under consideration. On the complex end of the spectrum, highly sophisticated models have been applied to study the intricacies of the interactions between the aerodynamics and mechanical dynamics of the vocal folds. Examples of these complex models are continuum mechanics models (Titze and Talkin 1979) and finite element models (AlipourHaghighi and Titze 1983). These models demand a great deal of computational power and are not conducive to simple cause and effect analysis. On the more simplistic end of the spectrum, lumped element models attempt to divide the vocal fold tissue into the largest possible portions such that the interactions between these portions and the aerodynamic forces can be studied with enough detail to investigate specific research questions. While the lumped element approach provides a coarse facsimile of the real, physical system, the small number of tunable parameters and computational efficiency provides important advantages over the finite element approach in some cases. In any case, the modeling approach must be sufficiently complex to investigate the research question at hand while minimizing the degrees of freedom of the system. Based on the 
objectives of this study, the lumped element approach was selected to model the vocal folds. An overview of several of these models is provided in the following chapter to lay the groundwork for the description of the model employed in this study.

In addition to the vocal fold model, an algorithm describing the trachea and vocal tract must be included to complete the representation of the vocal system. These parts of the system serve to shape the sound waves by acting as acoustic resonators, thereby filtering the component frequencies of the disturbance created by the glottal flow source. Several approaches to simulating the acoustic resonators can be identified in the literature, but the two main ones are: (1) the equivalent circuit model, and (2) the wave-reflection model. In essence, both approaches attempt to calculate the one-dimensional transfer of energy through the vocal tract and calculate the radiated sound. Typically, the vocal tract is divided into a series of discrete segments with various cross-sectional areas. The equivalent circuit model represents each segment as a simple electrical circuit, while the wave-reflection model applies the known solution to the onedimensional wave equation and calculates wave scattering at each segment junction. Both methods are described in more detail below.

An early lumped element model consisted of a single mass-spring system to represent one vocal fold as a self-oscillating source for input into the vocal tract (Flanagan and Landgraf 1968). The other vocal fold was represented as a mirror image of the first vocal fold resulting in a perfectly symmetrical right/left vocal fold scenario (bilateral symmetry). This one-mass model could not achieve a limit cycle unless an intertive vocal tract load was attached. On the contrary, experimental observations show that a vocal tract is not required for the oscillators to exhibit limit cycle behavior (Doellinger, Berry and Montegquin 2004). The main deficiency in the onemass model was that the vertical phase difference in the vocal folds during oscillation could not 
be achieved with a single degree of freedom. The vertical phase difference, or the equivalent, is required to induce the reversal of the driving force direction, which is essential for selfoscillation. More recently, an effective one mass model (EOMM) has been proposed that does achieve a limit cycle without a vocal tract or vertical phase difference (Fulcher, Scherer, Melnykov et al. 2006). The proper vocal fold dynamics are achieved in the EOMM through the application of aerodynamic forces that resemble those observed experimentally, as well as the concept of negative coulomb damping which is introduced to ensure that the driving forces are always in the same direction as the vocal fold velocity. Theses forces, however, are inputs to the model and therefore do not include the feedback mechanism between the vocal fold motion and the aerodynamic driving forces.

\subsubsection{The Two-Mass Model of Ishizaka and Flanagan (1972)}

The starting point for the lumped element model employed in this study is the two-mass model developed by Ishizaka and Flanagan (1972), hereafter referred to as the IF72 model. This model will be reviewed in detail since it forms the basic structure of the model applied in this study. In the IF72 model, one of the vocal folds is divided in thickness (inferior-superior depth) into two stiffness-coupled, mechanical oscillators that are driven by aerodynamic forces and are allowed only lateral displacement. The two masses are connected to a rigid wall (representing the thyroid cartilage) via non-linear springs and to each other with a linear spring. The oscillators are also subject to viscous resistance. A schematic of the IF72 model is provided in Figure 2-1.

The equations of motion for the coupled mechanical oscillators are:

$$
\begin{aligned}
& m_{1} \ddot{x}_{1}+r_{1} \dot{x}_{1}+s_{1}\left(x_{1}\right)+k_{c}\left(x_{1}-x_{2}\right)=F_{1}, \\
& m_{2} \ddot{x_{2}}+r_{2} \dot{x}_{2}+s_{2}\left(x_{2}\right)+k_{c}\left(x_{2}-x_{1}\right)=F_{2} .
\end{aligned}
$$




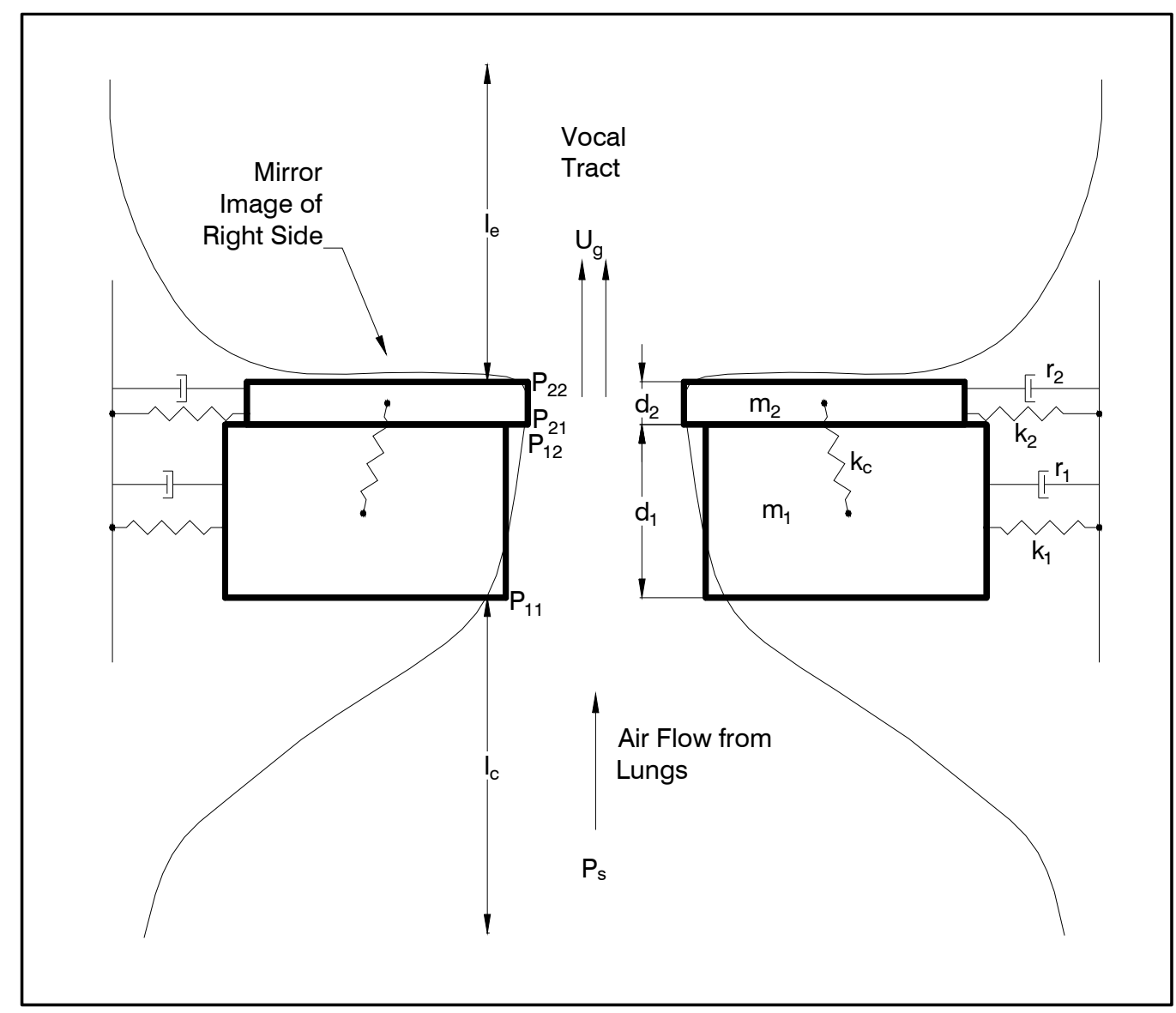

Figure 2-1 Schematic of the IF72 model of the vocal folds: side view (based on: Ishizaka and Flanagan 1972)

The first terms of equations 2.1 represent the inertia of the vocal folds segments, with $\mathrm{m}_{1}$ and $\mathrm{m}_{2}$ denoting the masses of the bottom and top parts of the folds, respectively. The second terms represent the viscous resistances associated with each segment, with damping constants $r_{1}$ and $r_{2}$. The third terms define the spring forces, or restoring forces, $s_{1}$ and $s_{2}$, which represent tissue stiffness. The spring functions are non-linear in accordance with stiffness measurements on fresh, excised human vocal folds (Ishizaka and Kaneko 1968). The fourth term in each equation is the coupling between the masses via a linear spring with constant $k_{c}$. The final term on the right hand side of each equation is the driving force. 
The coupling spring governs the flexibility of the vocal fold by dictating how energy is transferred between the masses. This effect is controlled by the elongation tension due to contraction of the cricothyroid muscles. The non-linear spring functions represent the tension of the vocal folds which is controlled by the mucosal tension and contraction level of the thyroarytenoid muscle. The IF72 model defines the restoring forces of the vocal folds separately under open and closed circumstances. It is argued that the vocal folds deform during collision in response to the contact forces. These contact forces may be viewed as increases in the effective restoring forces. Therefore, an additional non-linear restoring force is added. The restoring forces are given by,

$$
\begin{aligned}
& s_{i}\left(x_{i}\right)=k_{i}\left(x_{i}+\eta_{k i} x_{i}^{3}\right), \quad \text { (open glottis), } \\
& s_{i}\left(x_{i}\right)=k_{i}\left(x_{i}+\eta_{k i} x_{i}^{3}\right)+h_{i}\left[\left(x_{i}+x_{i c o l}\right)+\eta_{h i}\left(x_{i}+x_{i c o l}\right)^{3}\right], \text { (during collision), }
\end{aligned}
$$

where $\mathrm{i}=1,2$ for the two masses, $k_{i}$ are the spring constants for the open glottis condition, the $h_{i}$ are the spring constants for the closed glottis condition, the $\eta_{k i}$ are the nonlinearity coefficients for the open glottis condition, the $\eta_{h i}$ are the nonlinearity coefficients during collision, and the $x_{i c o l}$ are the locations of the masses at the moment of collision, which are always at the midline for a bilaterally symmetric model.

Similar to the restoring forces, the viscous resistances are defined for both open and closed glottis conditions. The resistance is increased during collision to represent the stickiness of the vocal folds in contact. The IF72 model represents the viscosity in terms of dimensionless damping ratios, $\zeta_{1}$ and $\zeta_{2}$,

$$
r_{1}=2 \zeta_{1} \sqrt{m_{1} k_{1}} \text { and } r_{2}=2 \zeta_{2} \sqrt{m_{2} k_{2}}
$$

During open glottis conditions the damping ratios were set at $\zeta_{1}=0.1$ and $\zeta_{2}=0.6$.

During collision, they were set above the critical damping values, $\zeta_{1}=(1.0+0.1)$ and $\zeta_{2}=(1.0+$ 
0.6 ), where critical damping would occur at $\zeta_{1}=\zeta_{2}=1$. As with most of the physiological parameters in the IF72 model, the damping values were selected on a trial and error basis to determine what mix of parameters resulted in "normal" vocal fold oscillation. There was sparse physiological data at the time, and that which was available was used as a starting point to guide selection of the other parameters. More on this will be discussed when the parameters of various models are compared below.

The coupled oscillators are driven by the aerodynamic forces $F_{1}$ and $F_{2}$ which are derived from the subglottal pressure $\left(\mathrm{P}_{\mathrm{s}}\right)$, the intraglottal pressures $\left(\mathrm{P}_{11}, \mathrm{P}_{12}, \mathrm{P}_{21}, \mathrm{P}_{22}\right)$, and the inlet pressure into the vocal tract (refer to Figure 2.1). As air flows from the trachea to the glottis it encounters a contraction in the cross-sectional area of the channel over a length $1_{\mathrm{c}}$. After the air flows through the glottal constriction, it encounters an expansion in the channel over a length $1_{\text {e }}$. The trachea and the vocal tract are assumed to be of circular cross-section. Within the glottis, the cross-sectional areas are assumed to be rectangular and are given by the time-dependent functions $\mathrm{A}_{\mathrm{g} 1}$ and $\mathrm{A}_{\mathrm{g} 2}$. These depend on the displacements, $\mathrm{x}_{1}$ and $\mathrm{x}_{2}$, of the masses $\mathrm{m}_{1}$ and $\mathrm{m}_{2}$, respectively. Due to the bilateral symmetry of the vocal folds, the glottal areas are simply the initial areas, $\mathrm{A}_{\mathrm{g} 01}$ and $\mathrm{Ag}_{\mathrm{g} 02}$, plus twice the area of each side, that is,

$$
\begin{aligned}
& A_{g 1}(t)=A_{g 01}+2 l_{g} x_{1}(t), \\
& A_{g 2}(t)=A_{g 02}+2 l_{g} x_{2}(t),
\end{aligned}
$$

where $1_{\mathrm{g}}$ is the length of the vocal fold in the posterior/anterior direction. A top view of the IF72 model vocal folds is presented in Figure 2-2.

One of the fundamental relations dictating fluid flow in all vocal system models is the Bernoulli equation, which is essentially a statement of energy conservation, not considering losses. If gravitational potential energy is not considered, the Bernoulli equation is, 


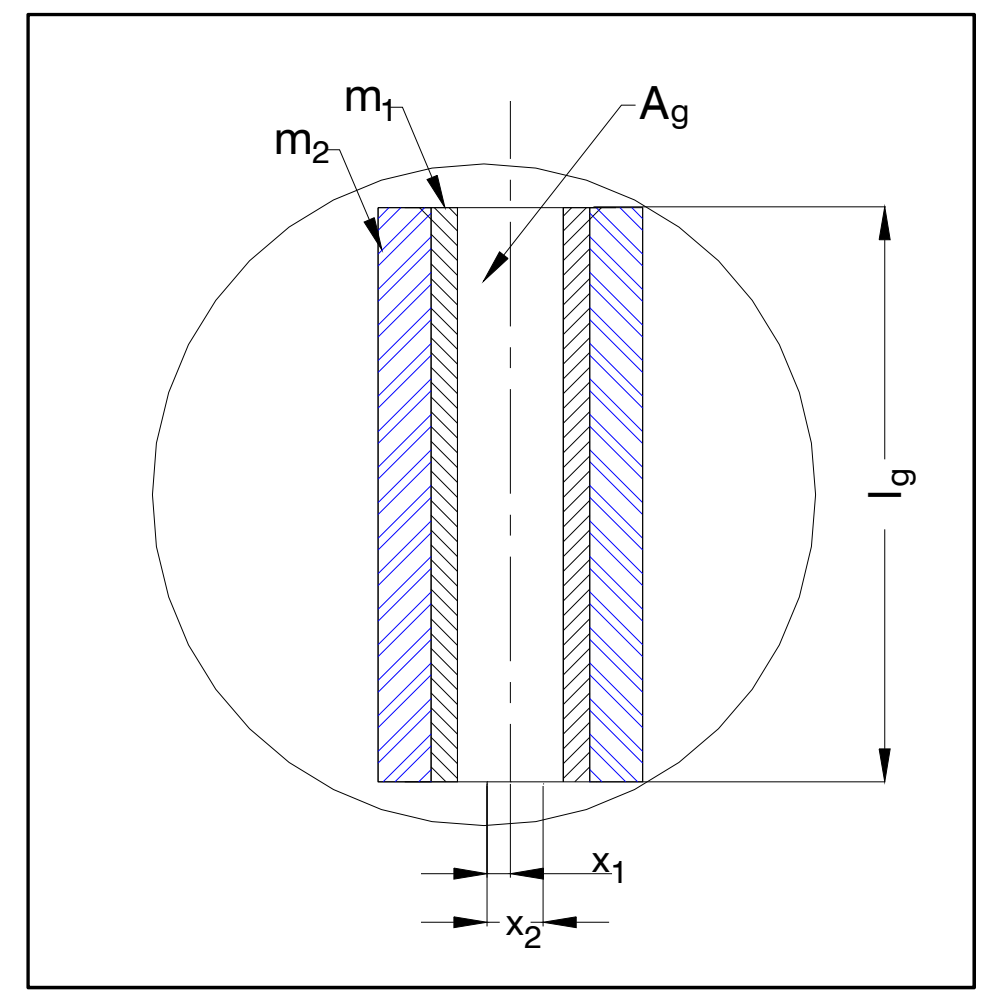

Figure 2-2 Schematic of the IF72 model vocal folds: top view (based on: Ishizaka and Flanagan 1972).

$$
P+\frac{1}{2} \rho v^{2}=\text { constant }
$$

where $P$ is the static pressure, $\frac{1}{2} \rho v^{2}$ is the kinetic pressure for velocity $v$ at a given point in the fluid, and $\rho$ is the density of air (van den Berg, Zantema and Doornenbal 1957). Assuming a constant airflow rate of $U=v A$, where $A$ is the area of the channel, the pressure differences therefore only depend on the changing areas of the channel. The application of the Bernoulli equation is limited by the various losses as well as the spatial and temporal changing geometry of the vocal system. For example, the contraction at the glottal entrance creates a region of stagnant air along the periphery of the channel which makes the area $A_{g 1}$ seem smaller than it actually is. Empirical studies indicate that the pressure drop in the contraction region is increased by an average additional factor of 0.37 (van den Berg, Zantema and Doornenbal 1957). This loss factor was applied in the IF72 model. The pressure decreases due to viscous losses within the 
glottis are based on the Poiseuille equation and the measurements of van den Berg et al. (1957) with the associated resistance,

$$
R_{v 1}=\frac{12 \mu l_{g} d_{1}}{A_{g 1}^{3}}
$$

where $R_{v 1}$ is the resistance to airflow in the first section of the glottis and $\mu$ is the shear viscosity of the air. A similar resistance is applied to the second (upper) section of the glottis. At the discrete area change between masses $m_{1}$ and $m_{2}$, the velocity changes but the flow remains continuous resulting in a pressure change,

$$
\Delta P_{\text {junction }}=\frac{1}{2} \rho U_{g}^{2}\left(\frac{1}{A_{g 2}^{2}}-\frac{1}{A_{g 1}^{2}}\right),
$$

where $U_{g}$ is the airflow through the glottis. At the glottal exit there is a pressure recovery which the IF72 model estimates based on momentum conservation to be,

$$
\Delta P_{r e c}=\frac{1}{2} \rho \frac{U_{g}^{2}}{A_{g 2}^{2}} \cdot 2 \frac{A_{g 2}}{A_{1}}\left(1-\frac{A_{g 2}}{A_{1}}\right)
$$

where $A_{1}$ is the cross-sectional area of the first section of the vocal tract. To complete the calculations of the pressure changes along the flow channel, the inertia of the air must also be considered. In the network theory of vocal transmission the inertia is represented by a series inductance given by $L=\rho l / A$, where $l$ is the length of the section and $A$ is the cross-sectional area (Fant 1960).

Based on the above pressure considerations and including the inertia of the air, the IF72 model describes the pressure distribution along the glottis as follows,

$$
\begin{aligned}
& P_{S}-P_{11}=1.37 \frac{\rho}{2}\left(\frac{U_{g}}{A_{g 1}}\right)^{2}+\int_{0}^{l_{c}} \frac{\rho}{A_{c}(x)} d x \frac{d U_{g}}{d t} \\
& P_{11}-P_{12}=\frac{12 \mu l_{g} d_{1}}{A_{g 1}^{3}} U_{g}+\frac{\rho d_{1}}{A_{g 1}} \frac{d U_{g}}{d t}
\end{aligned}
$$




$$
\begin{aligned}
& P_{12}-P_{21}=\frac{1}{2} \rho U_{g}^{2}\left(\frac{1}{A_{g 2}^{2}}-\frac{1}{A_{g 1}^{2}}\right), \\
& P_{21}-P_{22}=\frac{12 \mu l_{g} d_{2}}{A_{g 2}^{3}} U_{g}+\frac{\rho d_{2}}{A_{g 2}} \frac{d U_{g}}{d t}, \\
& P_{22}-P_{1}=-\frac{1}{2} \rho \frac{U_{g}^{2}}{A_{g 2}^{2}} \cdot 2 \frac{A_{g 2}}{A_{1}}\left(1-\frac{A_{g 2}}{A_{1}}\right) .
\end{aligned}
$$

The oscillators are driven by the forces which result from the mean pressures acting on the medial surfaces (the surface of the vocal folds exposed to the glottal opening). The mean pressures are calculated from equations (2.9) as follows:

$$
\begin{gathered}
P_{m 1}=\frac{1}{2}\left(P_{11}+P_{12}\right)=P_{s}-1.37 \frac{\rho}{2}\left(\frac{U_{g}}{A_{g 1}}\right)^{2}-\frac{1}{2}\left(\frac{12 \mu l_{g} d_{1}}{A_{g 1}^{3}} U_{g}+\frac{\rho d_{1}}{A_{g 1}} \frac{d U_{g}}{d t}\right), \\
P_{m 2}=\frac{1}{2}\left(P_{21}+P_{22}\right)=P_{m 1}-\frac{1}{2}\left[\left(\frac{12 \mu l_{g} d_{1}}{A_{g 1}^{3}}+\frac{12 \mu l_{g} d_{2}}{A_{g 2}^{3}}\right) U_{g}+\left(\frac{\rho d_{1}}{A_{g 1}}+\frac{\rho d_{2}}{A_{g 2}}\right) \frac{d U_{g}}{d t}\right]- \\
\frac{1}{2} \rho U_{g}^{2}\left(\frac{1}{A_{g 2}^{2}}-\frac{1}{A_{g 1}^{2}}\right) .
\end{gathered}
$$

The areas of the medial surfaces of masses $m_{1}$ and $m_{2}$ are given by $l_{g} d_{1}$ and $l_{g} d_{2}$, respectively. The average pressures of equation (2.10) apply only when air is allowed to flow through the glottis. Therefore, the IF72 model incorporates logical conditions to determine which forces should be applied at particular times in the cycle. The logical conditions are summarized in Table 2-1.

Since the intraglottal pressures depend on the areas of the glottis, the driving forces not only cause the oscillator motion, but the forces also depend on the displacement of the oscillators. Thus, the feedback mechanism between the aerodynamic forces and the mechanical oscillators is complete.

The IF72 model employs an equivalent network representation of the vocal system to combine the movement of air through the glottis and vocal tract. In this method, the resonator is 
Table 2-1 Force conditions for the IF72 model

\begin{tabular}{|c|c|c|c|}
\hline $\mathrm{x}_{\mathbf{1}}$ & $\mathrm{x}_{\mathbf{2}}$ & $\mathbf{F}_{\mathbf{1}}$ & $\mathbf{F}_{2}$ \\
\hline $\mathrm{x}_{1}>\mathrm{x}_{1 \mathrm{col}}$ & $\mathrm{x}_{2}>\mathrm{x}_{2 \text { col }}$ & $\mathrm{P}_{\mathrm{m} 1} \mathrm{l}_{\mathrm{g}} \mathrm{d}_{1}$ & $\mathrm{P}_{\mathrm{m} 2} \mathrm{l}_{\mathrm{g}} \mathrm{d}_{2}$ \\
\hline $\mathrm{x}_{1} \leq \mathrm{x}_{1 \mathrm{col}}$ & $\mathrm{x}_{2}>\mathrm{x}_{2 \text { col }}$ & $\mathrm{P}_{\mathrm{s}} 1_{\mathrm{g}} \mathrm{d}_{1}$ & 0 \\
\hline $\mathrm{x}_{1}>\mathrm{x}_{1 \text { col }}$ & $\mathrm{x}_{2} \leq \mathrm{x}_{2 \text { col }}$ & $\mathrm{P}_{\mathrm{s}} 1_{\mathrm{g}} \mathrm{d}_{1}$ & $\mathrm{P}_{\mathrm{s}} 1_{\mathrm{g}} \mathrm{d}_{2}$ \\
\hline $\mathrm{x}_{1} \leq \mathrm{x}_{1 \text { col }}$ & $\mathrm{x}_{2} \leq \mathrm{x}_{2 \text { col }}$ & $\mathrm{P}_{\mathrm{s}} 1_{\mathrm{g}} \mathrm{d}_{1}$ & 0 \\
\hline
\end{tabular}

Note: $\mathrm{x}_{1 \mathrm{col}}$ and $\mathrm{x}_{2 \mathrm{col}}$ represent the point of collision for the lower and upper masses, respectively. For the bilaterally symmetric IF72 model, this collision point is always the midline of the glottis.

divided into segments and the acoustic properties of each segment are represented by an equivalent resistor-capacitor-inductor circuit. This approach has been used in other aspects of acoustics (Fant 1960) to model sound through various types of acoustic resonators. Similar to the flow of electric current, energy transfer is assumed to be one-dimensional as the air flows through the tubes of varying cross-section. To complete the parallel between electrical current and airflow, the airflow is treated like electrical current flowing through a transmission line, while the pressure is similar to the voltage. The resistive losses, capacitance, and inductance (or inertia) are dependent on the geometry of the channel and the properties of the medium. The network representation of the IF72 model is depicted in Figure 2-3.

The terms $R_{k 1}$ and $R_{k 2}$ in Figure 2-3 are kinetic resistance terms where $R_{k 1}$ includes the contraction losses and the pressure change at the junction from equation (2.7), and $R_{k 2}$ includes the pressure recovery from equation (2.8). The cross-sectional areas of the vocal tract are $\mathrm{A}_{1}$, $A_{2} \ldots A_{n}$, and the lengths are $1_{1}, 1_{2} \ldots 1_{n}$, where $n$ is the number of tube segments, which are represented by loops in the network. The inductances and resistances in each loop are split into two halves, with one half before and the other after the capacitor in each loop. The half inductances are given by $L_{i}=\rho l_{i} /\left(2 A_{i}\right)$ and the capacitances are $C_{i}=l_{i} A_{i} /\left(\rho c^{2}\right)$, where $\mathrm{c}$ is 
the speed of sound in air. The half series resistances are given by $R_{i}=\left(S_{i} / A_{i}^{2}\right) \sqrt{\rho \mu \omega / 2}$,

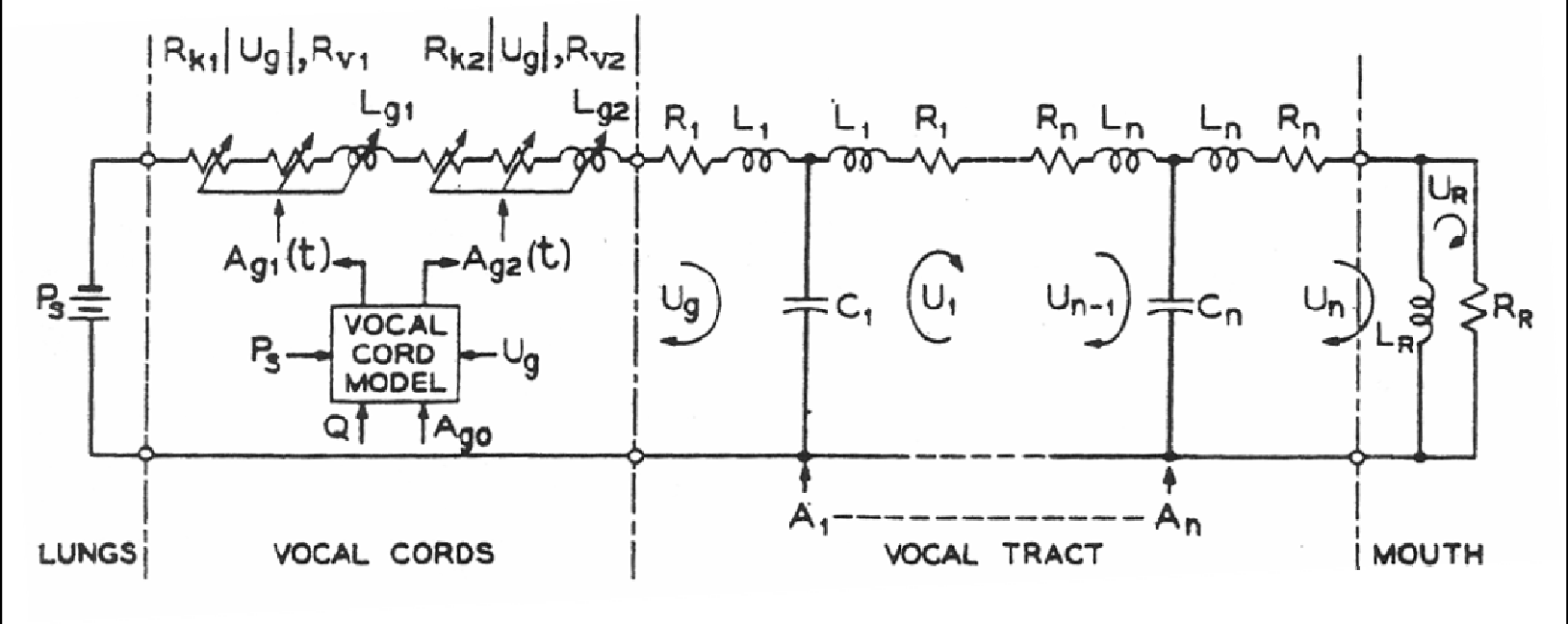

Figure 2-3 IF72 network representation of the glottis and vocal tract (source: Ishizaka and Flanagan 1972)

where $\mathrm{S}_{\mathrm{i}}$ is the circumference of the $\mathrm{i}^{\text {th }}$ tube, and $\omega$ is the radian frequency. In addition to the series resistance, an attenuation factor (ATT, ranging from 20 to 25) is used to increase the resistance beyond the viscous losses to account for losses due to heat and vibration of the resonator. The final loop represents the mouth and is a radiation load with inductance $L_{r}=$ $8 \rho /\left(3 \pi \sqrt{\pi A_{r}}\right)$ and the resistance is given by $R_{r}=128 \rho c /\left(9 \pi^{2} A_{r}\right)$, where $A_{r}$ is the final mouth area (Flanagan 1972).

The IF72 model divides a $16 \mathrm{~cm}$ long vocal tract into $\mathrm{n}=4$ segments of equal length. The area of each cylindrical tube segment is allowed to vary to simulate the shape of the vocal tract for various vowel sounds. A uniform cross-sectional area of $5 \mathrm{~cm}^{2}$ area was used to simulate the vowel sound $/ \ni /$, as in "ago". Based on the network representation given above and by applying Kirchoff's voltage law, the differential equations governing the airflow through the system are 


$$
\begin{aligned}
& \left(R_{k 1}+R_{k 2}\right)\left|U_{g}\right| U_{g}+\left(R_{v 1}+R_{v 2}\right) U_{g}+\left(L_{g 1}+L_{g 2}\right) \frac{d U_{g}}{d t}+L_{1} \frac{d U_{g}}{d t}+R_{1} U_{g}+ \\
& \quad \frac{1}{C_{1}} \int_{0}^{t}\left(U_{g}-U_{1}\right) d t-P_{s}=0, \\
& \left(L_{1}+L_{2}\right) \frac{d U_{1}}{d t}+\left(R_{1}+R_{2}\right) U_{1}+\frac{1}{C_{2}} \int_{0}^{t}\left(U_{1}-U_{2}\right) d t+\frac{1}{C_{1}} \int_{0}^{t}\left(U_{1}-U_{g}\right) d t=0, \\
& \left(L_{2}+L_{3}\right) \frac{d U_{2}}{d t}+\left(R_{2}+R_{3}\right) U_{2}+\frac{1}{C_{3}} \int_{0}^{t}\left(U_{2}-U_{3}\right) d t+\frac{1}{C_{2}} \int_{0}^{t}\left(U_{2}-U_{1}\right) d t=0, \\
& \left(L_{3}+L_{4}\right) \frac{d U_{3}}{d t}+\left(R_{3}+R_{4}\right) U_{3}+\frac{1}{C_{4}} \int_{0}^{t}\left(U_{3}-U_{4}\right) d t+\frac{1}{C_{3}} \int_{0}^{t}\left(U_{3}-U_{2}\right) d t=0, \\
& \left(L_{4}+L_{r}\right) \frac{d U_{4}}{d t}+R_{4} U_{4}-L_{r} \frac{d U_{r}}{d t}+\frac{1}{C_{4}} \int_{0}^{t}\left(U_{4}-U_{3}\right) d t=0, \\
& L_{r}\left(\frac{d U_{r}}{d t}-\frac{d U_{4}}{d t}\right)+R_{r} U_{r}=0 .
\end{aligned}
$$

The airflow, $U_{g}$, at each time step is used to calculate the driving forces, which cause the oscillators to move, thereby changing the glottal areas. The change in glottal area results in a new $U_{g}$ value and the process repeats itself. Therefore, the model involves simultaneously solving the eight nonlinear, ordinary, differential equations given by equations (2.1) and (2.11).

The IF72 model was successful in achieving some of observed aspects of speech production. For example, the model produced a phase difference of 55 degrees between the lower and upper mass, which provided a natural explanation for the lag of the upper surface of the vocal folds behind the lower surface, and an open quotient of approximately 0.6 under typical conditions, which were comparable to previous observations (Farnsworth 1940).

\subsubsection{The Asymmetric Two-Mass Model of Isshiki and Ishizaka (1976)}

The IF72 model was created for the purposes of artificial voice synthesis. However, it was later applied to the study of voice pathologies (Ishizaka and Isshiki 1976). In their 1976 work, Ishizaka and Isshiki extended the bilaterally symmetric IF72 model to an asymmetric model, which was employed to study the effects of tension imbalances between the right and left 
vocal folds. The imbalances were investigated by varying the $Q$ parameter, the prephonatory area, and the subglottal pressure. The Q parameter linearly scales down the masses and thicknesses of the vocal folds while scaling up the stiffness. The results indicated that vibrations occur in the following three distinct modes: (1) differences in phase and amplitude of the opposing vocal folds; (2) near periodic motion without glottal closure; and (3) dicrotic or tricrotic motion (two or three frequencies observed in the oscillator waveform), which may lead to a diplophonic sound (more than one pitch). An interesting and relevant point mentioned in passing in the Ishizaka 1976 paper was that the output of the asymmetric two-mass model was "incorporated into a dynamic vocal tract synthesizer with automatic turbulent noise generators" (Ishizaka and Isshiki 1976, p. 1198). This suggests that adding random noise to a simple model may provide more natural voiced sounds. The model applied in this study takes a similar approach by adding varying degrees of randomness in the system to investigate possible sources of jitter and shimmer, which also play roles in how natural the phonatory output sounds.

\subsubsection{The 16-mass Model of Titze (1973)}

Due to the limited number of degrees of freedom, the IF72 model was not capable of handling horizontal inhomogeneities such as vocal nodes or polyps, or higher frequency excitations (Titze 1973). Introducing more degrees of freedom creates a more natural sounding voice and allows for the study of the effects of various nonuniform structures. With these goals in mind, a 16-mass lumped element model was developed to enlarge the horizontal degrees of freedom and allow for motion in both the lateral direction and in the direction of flow (Titze 1973; Titze 1974). The 16-mass model was similar to the IF72 model but with 8 pairs of oscillators extending in the anterior/posterior direction. The upper masses, which represented the mucosa layer, were connected only to the lower masses, which represented the muscle and 
ligaments, and not to a rigid wall. The lower, larger masses were connected back to a rigid wall with nonlinear springs. Bilateral symmetry was maintained in the 16-mass model.

The driving forces applied in the 16-mass model were similar to those of the IF72 model with the exception that the forces were calculated at each of the eight pairs of oscillators. The flow through the glottis was also calculated in a similar manner. Further, pressure changes of the type described by equation 2.7 were considered at the boundary of each mass segment of the vocal folds. However, airflow and pressure transmission through the vocal tract were calculated using the wave-reflection method rather than the equivalent network method. An overview of the wave-reflection method is provided below.

\subsubsection{The Wave-Reflection Model of the Vocal Tract}

The wave-reflection method was first developed in 1962 (Kelly and Lochbaum) and has been applied in many voice simulation studies since (Liljencrants 1985; Story 1995). The objective of the wave-reflection technique is to approximate the propagation of the acoustic wave through the vocal tract. Since there is no simple solution to the wave equation for arbitrarily changing boundary conditions, the problem is simplified by dividing the vocal tract and nasal tract into tubes of arbitrary cross-section and equal length. The problem is further simplified by assuming one-dimensional wave propagation, which is mostly justified by the fact that $k r<1$ for most frequencies of interest, where $r$ is the average radius of the tract, $k=2 \pi / \lambda$ is the wave number, and $\lambda$ is the wavelength (Titze 1973). The method involves calculating the reflection and transmission coefficients of the wave at each tube junction at each step in time. The reflected and transmitted waves caused by scattering at each junction then become the waves incident on the adjacent junctions, after a time $\Delta t=\Delta x / c$, where $\Delta x$ is the length of a tube and c is the speed of sound. By applying the continuity of pressure and flow at each boundary, and the 
superposition principle, the incident and reflected portions of the waves can be added at each time step to determine the total pressure or flow at each junction. The process is conducted in time synchrony with the speed of propagation of the sound wave. The method also allows for wave propagation through multiple junctions, which may be formed where the vocal tract meets the nasal tract and mouth.

Several alternative methods have been proposed to incorporate energy losses in the wavereflection method. One method included a series resistor between consecutive tubes to induce an appropriate pressure drop (Milenkovic 1987). Other methods included frequency dependent viscous losses and losses due to the yielding walls of the vocal tract by replacing the constant resistance term by a unit delayed digital filter (Liljencrants 1985). More recently, the losses due to wall vibrations have been included in the wave-reflection model by adding an equivalent circuit at the junction of each tube (Story 1995).

\subsubsection{The Body-Cover Model of Story and Titze (1995)}

The work of Hirano (1974) suggests that the vocal folds should be considered as a double layer system with different mechanical properties; a body structure comprised of muscle fibers and ligaments, and a cover consisting of a flexible sheath that is loosely connected to the body. A lumped element model of the vocal folds was developed based on the body-cover structure (Story and Titze 1995). The body-cover model extended the IF72 model to include a third mass, which acted as the body. The body mass was coupled to the cover masses via nonlinear springs and the body was not subject to any external driving forces. The body mass was then connected back to the thyroid cartilage via a nonlinear spring. The addition of the third mass essentially added a third equation of motion to equations 2.1 and new coupling terms. A schematic of the body-cover model is provided in Figure 2-4. 


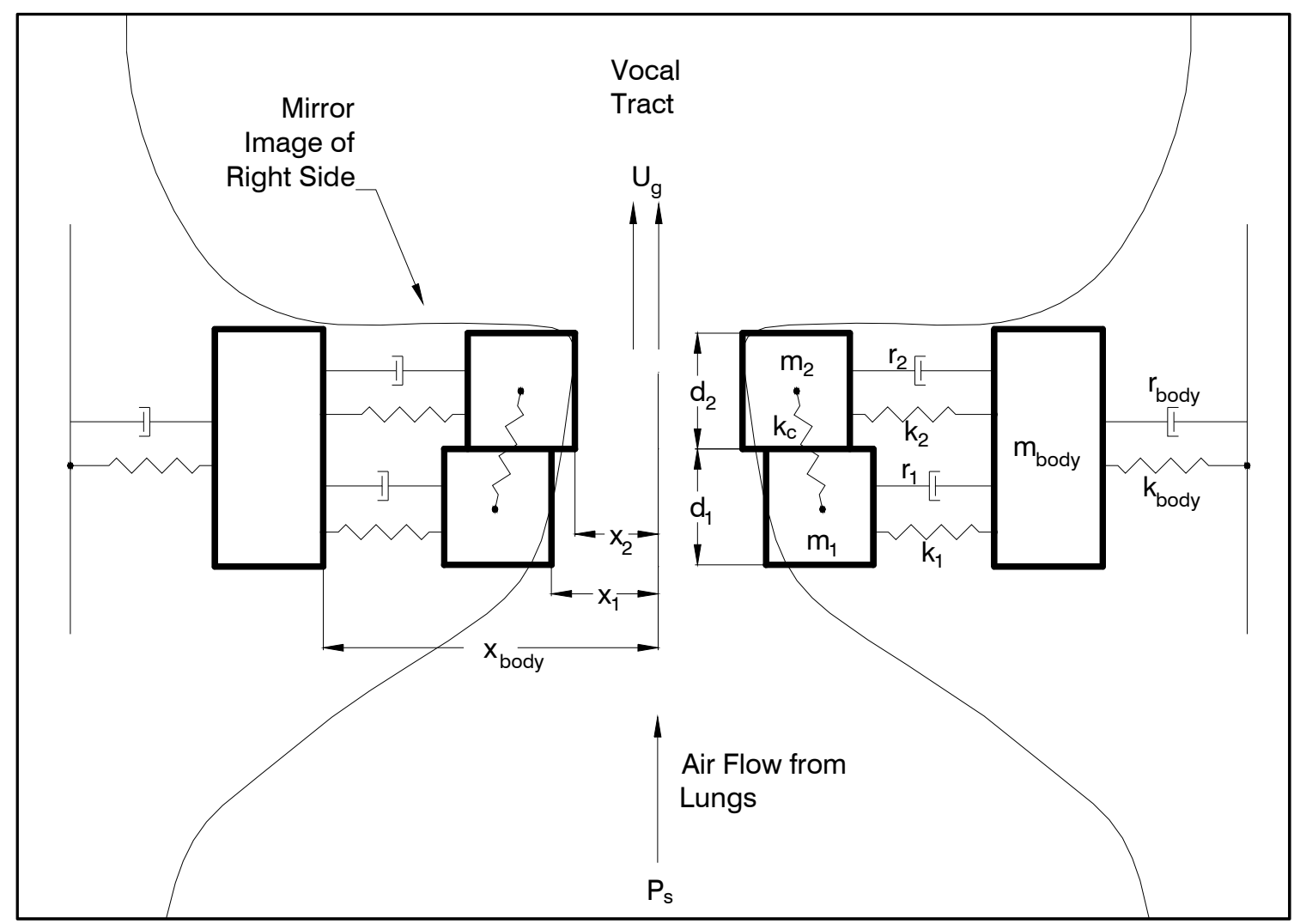

Figure 2-4 Schematic of the three-mass body-cover model (based on: Story and Titze 1995)

The body-cover model also differed from the IF72 model with respect to: (1) the driving forces; (2) the calculation of glottal flow; (3) the method used to simulate the vocal tract; and (4) the biomechanical parameters. The driving forces were based primarily on the Bernoulli effect with the addition of an air jet, which formed at specific vocal fold configurations. Due to the increased kinetic energy of the air stream at the narrow glottal opening, the airflow tends to pull away from the walls of the channel, forming a jet. The exact location of jet formation and its relationship to the glottal midline has been the subject of many studies (Scherer, Shinwari, De Witt et al. 2001; Scherer, Shinwari, De Witt et al. 2002; Thapa 2005; Neubauer, Zhang, Miraghaie et al. 2007). Jet formation is most likely during the divergent part of the cycle and is accompanied by vortex formation and turbulence within and downstream of the glottis. Since there were only two portions of glottal area to consider (one portion for the lower mass and one 
for the upper mass), the body-cover model assumed that the jet was formed at the end of the smaller of the two areas. Within the jet regime the net pressure was assumed to be constant. Losses were not considered in the pressure calculations, but the pressure recovery at the glottal exit was determined in accordance with the IF72 model as given in equation 2.8.

The glottal airflow rate in the body-cover model was calculated according to Titze (1984). The authors suggest that the resistance and inertance terms applied in the IF72 model are only valid for steady flow and constant geometry, stating that "they are based on steady flow between long parallel plates (Poiseuille flow) and were proposed by van den Berg (1957) for experimentation on static models" (Story and Titze 1995). However, the flow equation applied in the body-cover model includes a transglottal pressure coefficient, $\mathrm{k}_{\mathrm{t}}$ (Scherer and Titze 1983), which is also based on steady flow conditions in a static model. As of yet, there seems to be no consistent or standard method to deal with the intraglottal pressures and flow rates.

The trachea, vocal tract and nasal tract were simulated using the wave-reflection model described above with a uniform vocal tract area of $5 \mathrm{~cm}^{2}$. However, the radiation load was calculated in the same manner as the IF72 model. It is unclear how the airflow from the source was inserted into the wave reflection model since the sampling time for the wave-reflection model must be known in advance and the frequency of vocal fold oscillation is typically an output of the model. It seems that the sampling time of the wave-reflection model would have to match the fundamental period of the vocal fold oscillations, which is not known in advance. The procedure is not specified in the Story and Titze (1995) paper.

The biomechanical parameters in the body cover model were based on information that was not available at the time of the IF72 model. Consequently, many of the parameters are different. Some of these differences are summarized in Table 2-2. 
Table 2-2 Comparison of biomechanical parameters in the IF72 model and the body-cover model

\begin{tabular}{|l|l|l|l|}
\hline \multicolumn{1}{|c|}{ Parameter } & \multicolumn{1}{c|}{ Description } & \multicolumn{1}{c|}{ IF72 Model } & \multicolumn{1}{c|}{ Body-Cover Model } \\
\hline $\mathrm{m}_{1}$ & lower mass & $0.125 \mathrm{~g}$ & $0.01 \mathrm{~g}$ \\
\hline $\mathrm{m}_{2}$ & upper mass & $0.025 \mathrm{~g}$ & $0.01 \mathrm{~g}$ \\
\hline $\mathrm{m}_{\text {body }}$ & body mass & Not Applicable & $0.05 \mathrm{~g}$ \\
\hline $\mathrm{k}_{\mathrm{c}}$ & $\begin{array}{l}\text { upper/lower coupling } \\
\text { spring constant }\end{array}$ & 25,000 dyne $/ \mathrm{cm}$ & $500-2,000 \mathrm{dyne} / \mathrm{cm}$ \\
\hline $\mathrm{k}_{1}$ & lower spring constant & 80,000 dyne $/ \mathrm{cm}$ & 5,000 dyne $/ \mathrm{cm}$ \\
\hline $\mathrm{k}_{2}$ & upper spring constant & 8,000 dyne $/ \mathrm{cm}$ & 3,500 dyne $/ \mathrm{cm}$ \\
\hline $\mathrm{k}_{\text {body }}$ & body spring constant & Not Applicable & $20,000-700,000$ dyne/cm \\
\hline$\zeta_{1}$ (open glottis) & lower damping ratio & 0.1 & 0.4 \\
\hline$\zeta_{2}$ (open glottis) & upper damping ratio & 0.6 & 0.4 \\
\hline$\zeta_{1}$ (closed glottis) & lower damping ratio & 1.1 & 0.8 \\
\hline$\zeta_{2}$ (closed glottis) & upper damping ratio & 1.6 & 0.8 \\
\hline$\zeta_{\mathrm{b}}$ & body damping ratio & Not Applicable & 0.2 \\
\hline $\mathrm{d}_{1}$ & lower mass thickness & $0.25 \mathrm{~cm}$ & $0.15 \mathrm{~cm}$ \\
\hline $\mathrm{d}_{2}$ & upper mass thickness & $0.05 \mathrm{~cm}$ & $0.15 \mathrm{~cm}$ \\
\hline$l_{\mathrm{g}}$ & mass length & $1.4 \mathrm{~cm}$ & $1.0 \mathrm{~cm}$ \\
\hline $\mathrm{x}_{01}$ & $\begin{array}{l}\text { upper prephonatory } \\
\text { position (typical) }\end{array}$ & $0.018 \mathrm{~cm}$ & $0.018 \mathrm{~cm}$ \\
\hline $\mathrm{x}_{02}$ & $\begin{array}{l}\text { lower prephonatory } \\
\text { position (typical) }\end{array}$ & $0.018 \mathrm{~cm}$ & $0.0179 \mathrm{~cm}$ \\
\hline $\mathrm{x}_{0 \text { body }}$ & $\begin{array}{l}\text { body prephonatory } \\
\text { position }\end{array}$ & Not Applicable & $0.30 \mathrm{~cm}$ \\
\hline
\end{tabular}

Sources: (Ishizaka and Flanagan 1972; Story and Titze 1995)

In the 1995 paper, Story and Titze claim that one of the deficiencies of the IF72 model is that there is no direct physiological correlation between the spring stiffness and the effects of muscle contraction (Story and Titze 1995). However, Ishizaka and Flanagan state that the effects of the cricothyroid muscle are controlled by the coupling spring and the thyroarytenoid muscle by the nonlinear springs in the IF72 model (Ishizaka and Flanagan 1972). If this deficiency in the IF72 model is real however, then the 16-mass model must suffer from the same shortcoming. The body-cover model was developed to remedy this deficiency by incorporating additional coupling terms between the cover masses and the body mass. Story and Titze suggested that the body-cover model facilitates the study of the relative activations of the thyroarytenoid and 
cricothyroid muscles. Recent studies have been conducted with the body-cover model to study the effects of the relative muscle activations on fundamental frequency and aerodynamic quantities (Lowell and Story 2006). A comparison of the parameters that control the cricothyroid and thyroarytenoid muscles for the IF72 and body-cover models is presented in Figure 2-5.

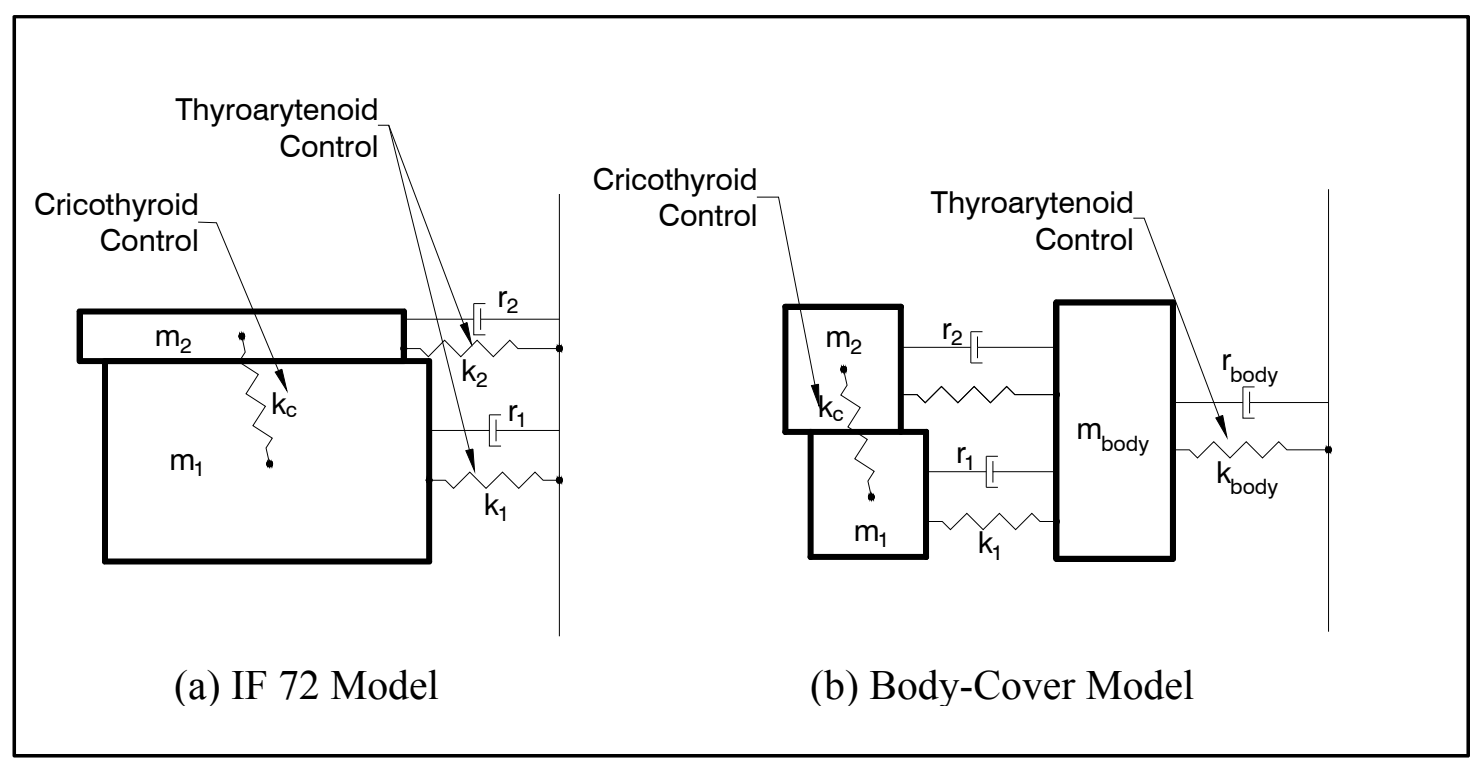

Figure 2-5 Muscle control parameters for (a) the IF72 model and (b) the body-cover model

\subsection{Overview of Voice Perturbation Measures}

In diagnosing and treating voice disorders, speech pathologists and otolaryngologists attempt to measure and reverse the cause of hoarse voice to restore a more natural sounding voice in patients. Unsteadiness or perturbations of acoustic characteristics such as fundamental frequency and amplitude provide valuable and readily attainable information regarding the degree and, possibly, the cause of voice disorders. As stated in the Introduction, "frequency (or period) perturbation - commonly called jitter - is the variability of the fundamental frequency (or, reciprocally, the fundamental period) from one cycle to the next" (Baken and Orlikoff 2000, p. 190). A similar measure is shimmer, or amplitude variations from one cycle to the next. There 
is a considerable body of literature indicating that increased jitter and shimmer are measured in patients with vocal and laryngeal pathologies (Baken and Orlikoff 2000) but the relationship of these measures to specific abnormalities remains unclear (Hirano, Hibi, Yoshida et al. 1988). According to Baken and Orlikoff (2000), possible causes of jitter are:

1) Neurogenic - related to the "twitching" contractile status of the muscle, i.e. random deviations from a steady-state muscle tension;

2) Aerodynamic - jets of air leaving the glottis may attach unpredictably to one wall or the other causing turbulent regions of varying intensity;

3) Mechanical - deviations in the biomechanical properties of the vocal folds can be associated with pathology (Jiang, Titze, Wexler et al. 1994), or varying stiffness of the vocal fold vascular bed due to the heartbeat;

4) Stylistic - such as artistic vibrato; and

5) Chaotic - a number of studies suggest that the vocal system may be chaotic and that the methods of nonlinear dynamics are an important tool in the study of voice disorders (Steinecke and Herzel 1995; Zhang, McGilligan, Zhou et al. 2004; Tokuda and Herzel 2005; Jiang, Zhang and McGilligan 2006; Rahn, Chou, Jiang et al. 2007).

While possible causes of shimmer are not as well understood as the causes of jitter, both measures are important and widely used diagnostic tools for clinicians. Unfortunately, there appears to be no universal method for quantifying jitter and shimmer. There are numerous indices available, most of which are discussed or referenced in Baken and Orlikoff (2000). Pinto and Titze (1990) attempted to unify the perturbation measures by suggesting that (1) frequency, rather than period, measures be used to quantify jitter; (2) various orders of statistical measures be employed rather than simply the first order root mean squared or mean rectified-centroid of 
the histogram; and (3) the terminology involved should be condensed. However, analyzing the frequency and amplitude data graphically appears to be most useful for detecting abnormalities and trends (Baken and Orlikoff 2000). Perhaps a review of the graphical analysis in each case could determine the most appropriate numerical indices depending on the specific situation. The approach applied in this study is mainly graphical with two simple indices.

The lumped element model approach may be advantageous for studying the causes of jitter and shimmer because the physical parameters can be modified separately in either a timedependent or time-independent manner. Also, due to the relative simplicity of the model, randomness can be added in realms of the unknown or unpredictable. On the other hand, the simplicity of the lumped element model may be a drawback since truly asymmetric driving forces are not attainable and additional degrees of freedom may play an important role. However, these are deficiencies that can be remedied in the future. 


\section{CHAPTER 3. THE MODEL}

The lumped element model employed in this study is based on the IF72 model. Attempts at using the body-cover model of Story and Titze (1995) as the basis for this study proved to be unsuccessful. Even attempts to modify the physical parameters of the IF72 model based on the references cited in the Story and Titze (1995) paper did not yield sufficient results. Specifically, the stability of the model could not be maintained over a wide variety of input parameters, and onset times characteristic of typical phonation were difficult to reproduce. The IF72 model showed good flexibility and versatility and it therefore formed the foundation for the model employed here. It is possible that if additional effort at applying the body-cover model in this setting is put forth, one may have more success. Regardless, the IF72 model seemed to be the most appropriate starting point for this study. Future work should entail the application of more sophisticated models to study voice perturbations and other effects of asymmetry.

The following modifications were made to the IF72 model to develop the model employed in this study:

1) The condition of bilateral symmetry was removed through the addition of an independent pair of coupled oscillators. This resulted in opposing pairs of coupled oscillators on either side of the glottal midline.

2) The driving forces were based on a combination of modified IF72 model forces and the pressure distribution data obtained from the experimental M5 apparatus.

3) Although the same model for the vocal tract was applied here as in the IF72 model, the equations were converted to second-order, ordinary differential equations (rather than first-order), for added uniformity in the computations. 
4) Several of the constant parameters in the IF72 model were changed to time-dependent functions to facilitate testing of the hypotheses.

5) The feedback between the vocal folds and the vocal tract was not applied in this model to simplify the application of the M5 data. Consequently, the model employed herein represents an excised larynx. Subsequent studies will incorporate the feedback mechanism to investigate the effects of source/filter interaction.

The details and consequences of these modifications are described in the following subsections.

\subsection{Bilateral Asymmetry}

In order to make the mechanical oscillators bilaterally asymmetric, several more parameters and two additional equations of motion (compared to equations 2.1) were required. In addition, since the midline of the glottis was no longer the only possible point of collision, the frame of reference for the system of oscillators was modified to allow for a more general coordinate frame. Specifically, the glottal midline was used as the $x=0$ line in this model. The set of equations describing the motion of both vocal folds is now given by,

$$
\begin{aligned}
& m_{R 1} \ddot{x}_{R 1}+r_{R 1} \dot{x}_{R 1}+s_{R 1}\left(x_{R 1}\right)+k_{R c}\left[\left(x_{R 1}-x_{R 10}\right)-\left(x_{R 2}-x_{R 20}\right)\right]=F_{R 1}, \\
& m_{R 2} \ddot{x}_{R 2}+r_{R 2} \dot{x}_{R 2}+s_{R 2}\left(x_{R 2}\right)+k_{R c}\left[\left(x_{R 2}-x_{R 20}\right)-\left(x_{R 1}-x_{R 10}\right)\right]=F_{R 2}, \\
& m_{L 1} \ddot{x}_{L 1}+r_{L 1} \dot{x}_{L 1}+s_{L 1}\left(x_{L 1}\right)+k_{L c}\left[\left(x_{L 1}-x_{L 10}\right)-\left(x_{L 2}-x_{L 20}\right)\right]=-F_{L 1}, \\
& m_{L 2} \ddot{x}_{L 2}+r_{L 1} \dot{x}_{L 1}+s_{L 1}\left(x_{L 1}\right)+k_{L c}\left[\left(x_{L 2}-x_{L 20}\right)-\left(x_{L 1}-x_{L 10}\right)\right]=-F_{L 2},
\end{aligned}
$$

where the subscripts " $R$ " and " $L$ " represent the right and left vocal folds, respectively and the subscripts "1" and "2" represent the lower and upper masses, respectively. The $m_{i j}$ terms represent the masses of the oscillators. Since the midline of the glottis was used as the origin of the frame of reference applied in this model, the $x_{i j}$ terms represent the displacements of the oscillators away from the midline, while the $\dot{x}_{i j}$ and $\ddot{x}_{i j}$ terms are the velocities and accelerations 
of the oscillators, respectively. The terms $x_{i j 0}$ are the initial positions of the oscillators. The viscous damping, restoring force functions, and the linear spring coupling terms are given by $r_{i j}$, $s_{i j}\left(x_{i j}\right)$, and $k_{i c}$, respectively. Finally the driving forces are the $F_{i j}$ terms on the right-hand side. Many of the pertinent parameters are identified in Figure 3-1.

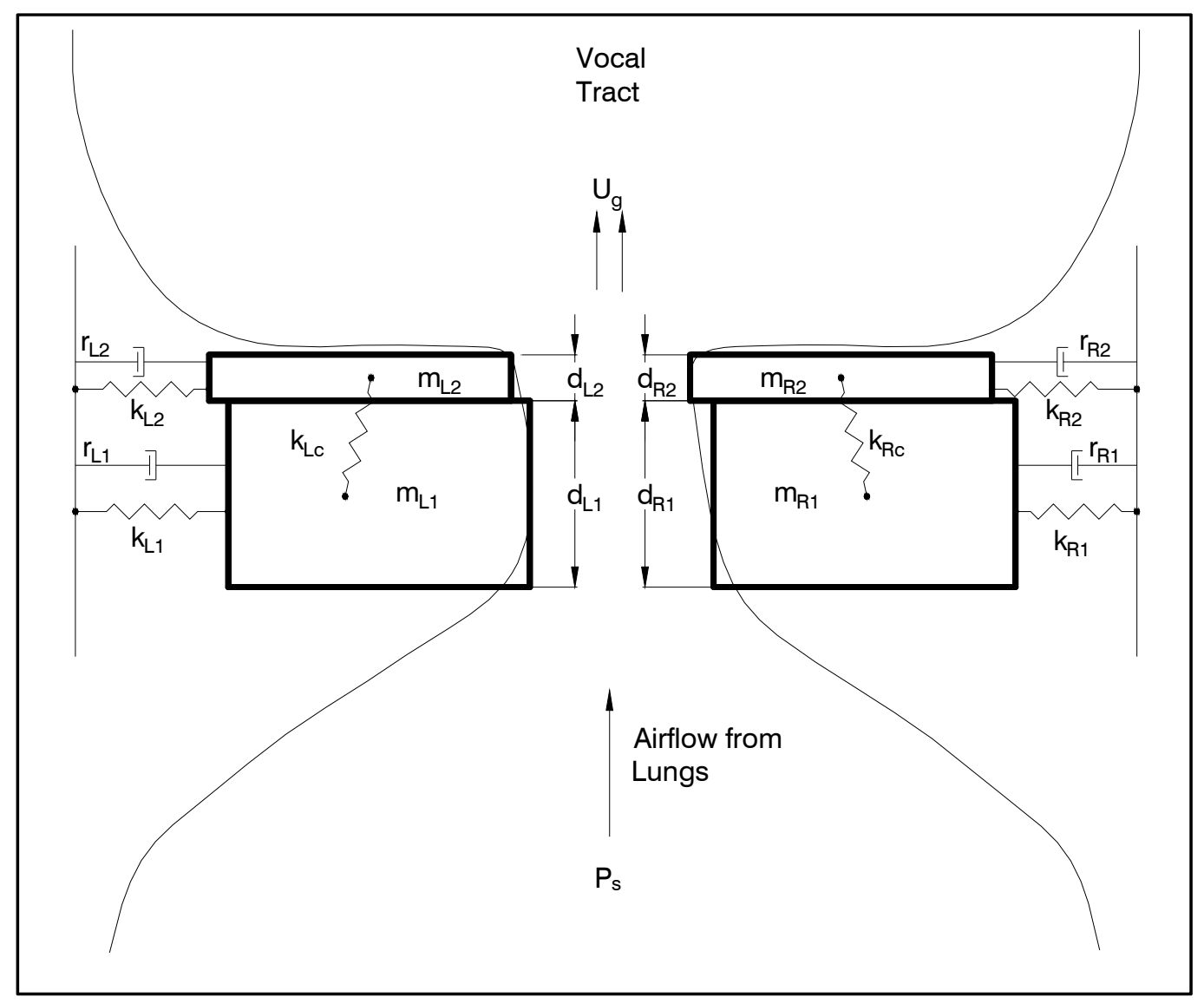

Figure 3-1 Schematic of the asymmetric two-mass model

Similar to the IF72 model, the spring forces in this model vary depending on whether the glottis is open or not. The differences in this model are that four spring functions are required instead of two (refer to equation 2.2), and the point of collision must be calculated since it is no longer a constant. The spring force functions are

$$
s_{i j}\left(x_{i j}\right)=k_{i j}\left[\left(x_{i j}-x_{i j 0}\right)+\eta_{k i j}\left(x_{i j}-x_{i j 0}\right)^{3}\right], \quad \text { (open glottis), }
$$




$$
\begin{aligned}
s_{i j}\left(x_{i j}\right)= & k_{i j}\left[\left(x_{i j}-x_{i j 0}\right)+\eta_{k i j}\left(x_{i j}-x_{i j 0}\right)^{3}\right]+ \\
& h_{i j}\left[\left(x_{i j}-x_{j c o l}\right)+\eta_{h i j}\left(x_{i j}-x_{j c o l}\right)^{3}\right], \text { (during collision), }
\end{aligned}
$$

where, as in equation 3.1, $i$ represents "R" or "L" for the right or left vocal fold, and $j$ represents " 1 " or " 2 " for the lower or upper oscillator, respectively. The $k_{i j}$ terms are the spring constants during the open glottis condition and the $h_{i j}$ terms are the spring constants during the closed glottis condition, representing the stiffness of the oscillators. The $\eta_{k i j}$ represent the nonlinearity coefficients during the open glottis condition, and the $\eta_{h i j}$ are the nonlinearity coefficients during the closed glottis condition. The initial positions of the oscillators relative to the glottal midline are given by the $x_{i j 0}$ terms, and the displacements are given by the $x_{i j}$ terms. For the purposes of this model, the point of collision for each oscillator, $x_{i j c o l}$, was estimated as the midpoint between the colliding oscillators during the closed part of each cycle. Specifically, during the collision phase of the cycle, the point of collision is given by:

$$
\begin{aligned}
& x_{1 \mathrm{col}}=\frac{1}{2}\left(x_{R 1}+x_{L 1}\right) \text { for } x_{R 1}<x_{L 1} ; \\
& x_{2 \mathrm{col}}=\frac{1}{2}\left(x_{R 2}+x_{L 2}\right) \text { for } x_{R 2}<x_{L 2} .
\end{aligned}
$$

The simplifying assumption made in equation 3.3 is that the right and left oscillators surpass each other by the same distance after collision. This is not always the case in an asymmetric system but it serves as a reasonable approximation.

The viscous damping terms are identical to the IF72 model with the addition that all four oscillators are now subject to damping. Viscous damping for open and closed glottis conditions are handled in the same way as discussed in Chapter 2. 


\subsection{The Driving Forces}

The driving forces applied in this model are based on a combination of a modified version of the IF72 driving forces and empirical data collected from the experimental apparatus M5. The modifications of the IF72 driving forces were based on semi-quantitative information obtained from the M5 data. Therefore, a preliminary discussion of M5 is in order.

The purpose of the M5 model is to facilitate the measurement of pressures and airflow rates that are representative of actual pressures and flow rates in the human larynx. As mentioned in Chapter 1, M5 is a Plexiglas, static model of a typical human male larynx that is scaled up by a factor of 7.5. It is referred to as a static model because the vocal folds do not vibrate. The apparatus is comprised of a rectangular wind tunnel, removable vocal fold pieces to consider varying geometrical shapes, and sixteen pressure taps that are judiciously located to facilitate the measurement of pressure distributions. Fourteen of the pressure taps are located along the glottal surface. The removable vocal fold pieces vary in shape to allow for the study of various configurations that can be either bilaterally symmetric or asymmetric. A threedimensional schematic of M5 is presented in Figure 3-2. The vocal fold geometry is presented in Figure 3-3.

Many of the details regarding the apparatus, methodology, and results are provided in the published literature (Scherer, Shinwari, De Witt et al. 2001; Scherer, Shinwari, De Witt et al. 2002; Thapa 2005). Most of the information obtained from M5 that is relevant to this study is based on unpublished results available through the Department of Communication Disorders at Bowling Green State University (Scherer).

The pressure distributions were measured with respect to four independent variables. Specifically, pressure was measured as a function of subglottal pressure $\left(P_{s}\right)$, minimal glottal 


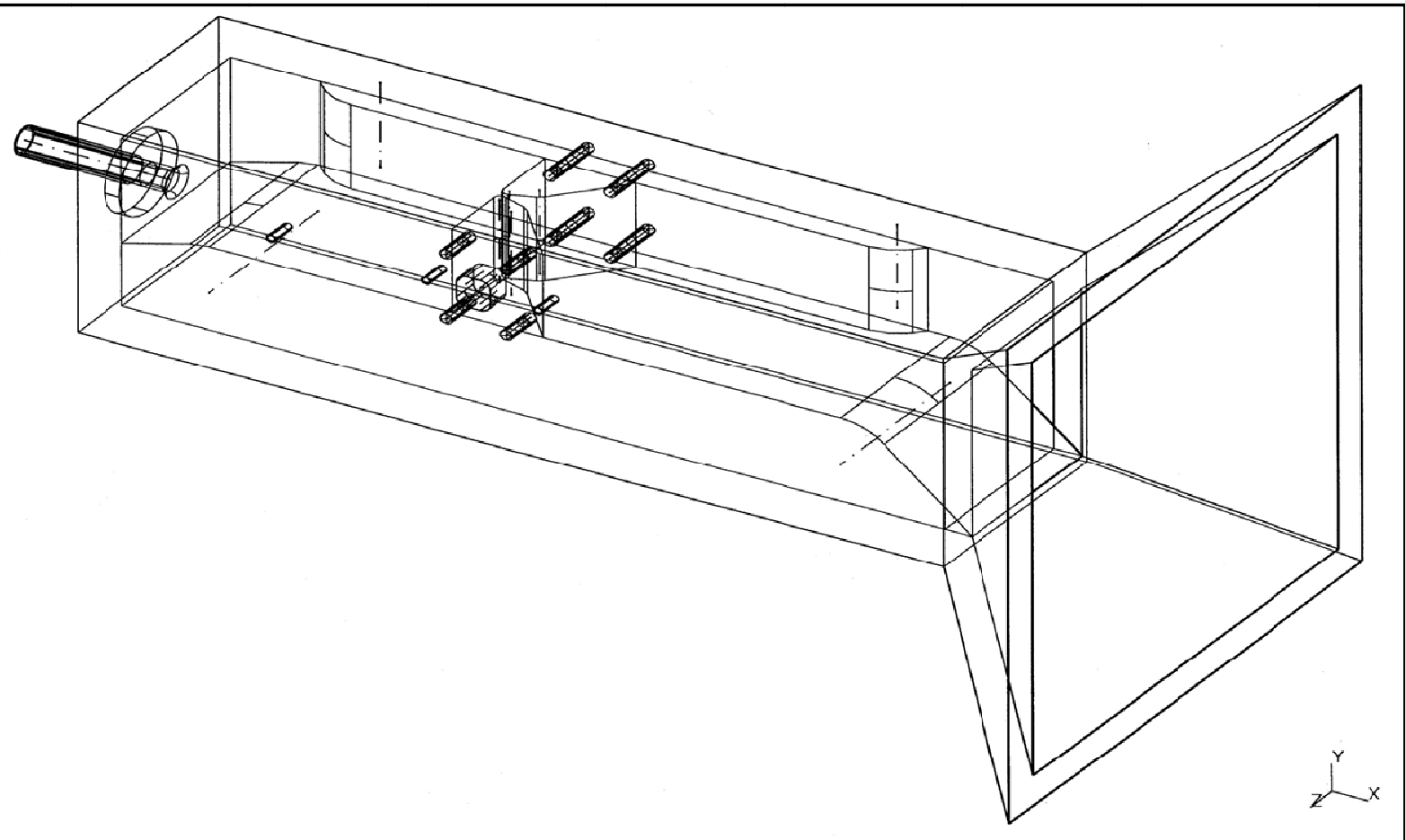

Figure 3-2 Schematic of the M5 apparatus (source: Scherer and Shinwari 2001) diameter $\left(d_{\text {min }}\right)$, included angle $(\phi)$ and distance in the direction of airflow $(z)$, that is, $P=$ $P\left(P_{s}, d_{\text {min }}, \phi, z\right)$. The included angle is given in Figure 3-3 as the variable $\Psi$. It is twice the angle that the glottal wall makes with the midline in the symmetric case.

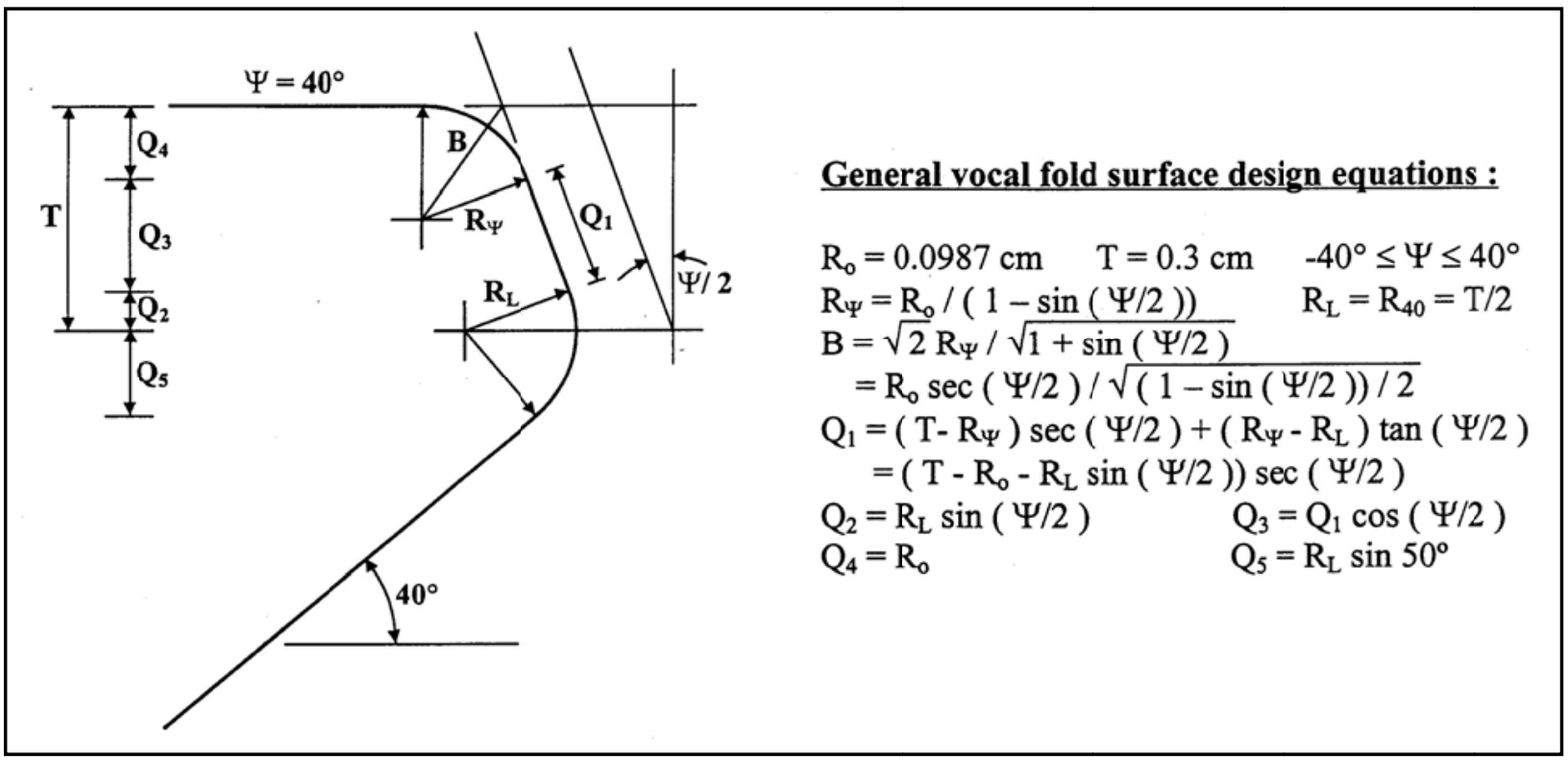

Figure 3-3 Schematic of vocal fold geometry (source: Scherer et al. 2001) 
A pressure asymmetry is typically observed for many glottal configurations due to the formation of a glottal air jet which flows nearer one vocal fold than the other. The side upon which the jet flows is referred to as the flow wall (FW) while the other side is referred to as the non-flow wall (NFW). Consequently, in any given M5 experiment, pressure measurements are collected for both FW and NFW. A convergent glottis sample of M5 data is provided in Figure 3-4 where the entrance and exit of the glottis are indicated by the dashed lines.

The M5 data employed in this study were based on pressure measurements collected for both the FW and NFW sides with symmetric vocal fold configurations at subglottal pressures of

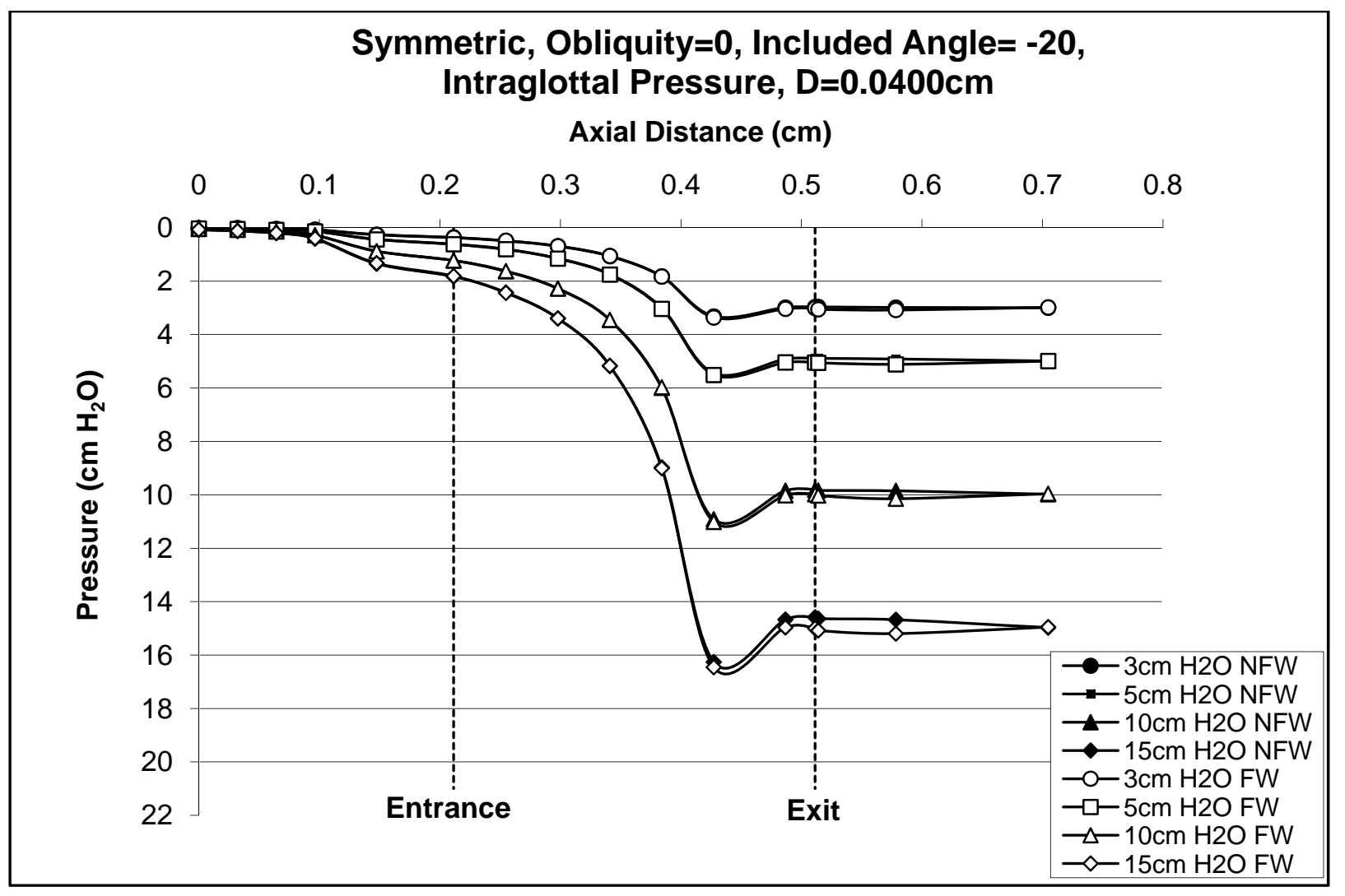

Figure 3-4 Sample of M5 experimental data for a converging angle of $-20^{\circ}$ (source: Scherer) $3,5,10,15$, and $25 \mathrm{~cm} \mathrm{H}_{2} 0$, minimal glottal diameters of $0.005,0.01,0.02,0.04,0.08,0.16$, and $0.32 \mathrm{~cm}$, and included angles of $-40,-20,-10,-5,0,5,10,20$, and 40 degrees, where convergent 
vocal fold configurations were designated as negative angles and divergent configurations were designated as positive angles. In all, there were approximately 9,000 data points.

The total M5 data set was truncated to establish a uniform grid of data points to facilitate numerical manipulation using the software Mathematica. The truncated data were inputs to Mathematica which was used to convert the discrete data points into a linear interpolating function in four variables. The final pressure distribution function, $P=P\left(P_{s}, d_{\text {min }}, \phi, z\right)$, had continuous ranges of subglottal pressure from 3 to $15 \mathrm{~cm} \mathrm{H}_{2} \mathrm{O}$, minimal glottal diameter from 0.005 to $0.16 \mathrm{~cm}$, included angles from $-40^{0}$ to $40^{\circ}$, and axial distances from 0 to $0.71 \mathrm{~cm}$. A sample of the interpolating function data for the case of $P_{S}=8 \mathrm{~cm} \mathrm{H}_{2} \mathrm{O}=7840 \mathrm{dyne} / \mathrm{cm}^{2}$ and $d_{\min }=0.04 \mathrm{~cm}$ is given in graphical form in Figure 3-5.

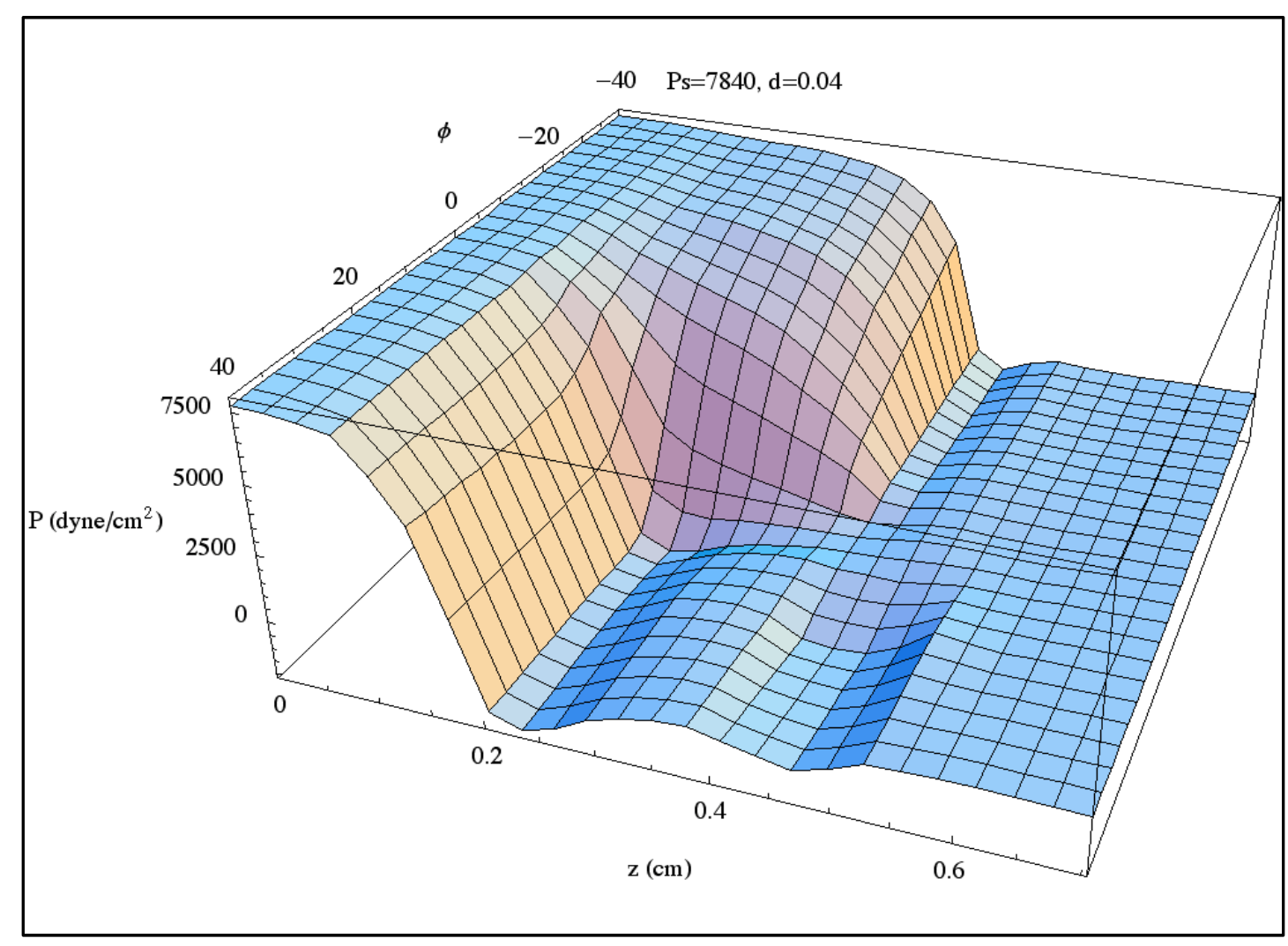

Figure 3-5 Sample of M5 interpolation function data in graphical form (Mathematica output). The glottal entrance is located at $\mathrm{z}=0.2114 \mathrm{~cm}$, and the glottal exit is located at $\mathrm{z}=0.5114 \mathrm{~cm}$. 
The interpolating function provides the value of the intraglottal pressure as a continuous function of the axial distance, $z$. A consequence of the lumped element model is that the pressure distribution must be averaged over the area of the vocal fold surface so that a driving force can be calculated for each oscillator. For example, although detailed pressure information is available over the entire thickness of mass $m_{R 1}$, it is the average pressure value that is multiplied by the surface area to calculate the driving force $F_{R 1}$. Therefore, much of the detail of the real system is sacrificed for the simplicity of the lumped element model.

Another approximation is due to the bilateral asymmetry requirement of the model applied in this study. Specifically, the included angle was required to be the sum of the angles on the right and left side rather than simply twice the angle on one side. That is, $\phi=\phi_{R}+\phi_{L}$, where $\phi_{R}$ is the angle of the right vocal fold and $\phi_{L}$ is the angle of the left vocal fold. A schematic is provided in Figure 3-6 to clarify the definition of these variables. Neither the M5 data applied here nor the IF72 driving forces account for asymmetric glottal configurations. In fact, an applicable theoretical model for the intraglottal pressures for asymmetric configurations is not available. The symmetric force equations applied here serve as a first approximation. There have been several M5 experiments conducted for asymmetric configurations (Scherer, Shinwari, De Witt et al. 2001; Scherer, Shinwari, De Witt et al. 2002; Thapa 2005) but, as of yet, the body of data is not sufficient to develop the required numerical or analytical formulas. The model employed here can be further refined by applying asymmetric pressure data as it becomes available.

Once the interpolating function was established, the pressure at any point along the vocal fold surface could be determined for any configuration within the range of the function variables. 


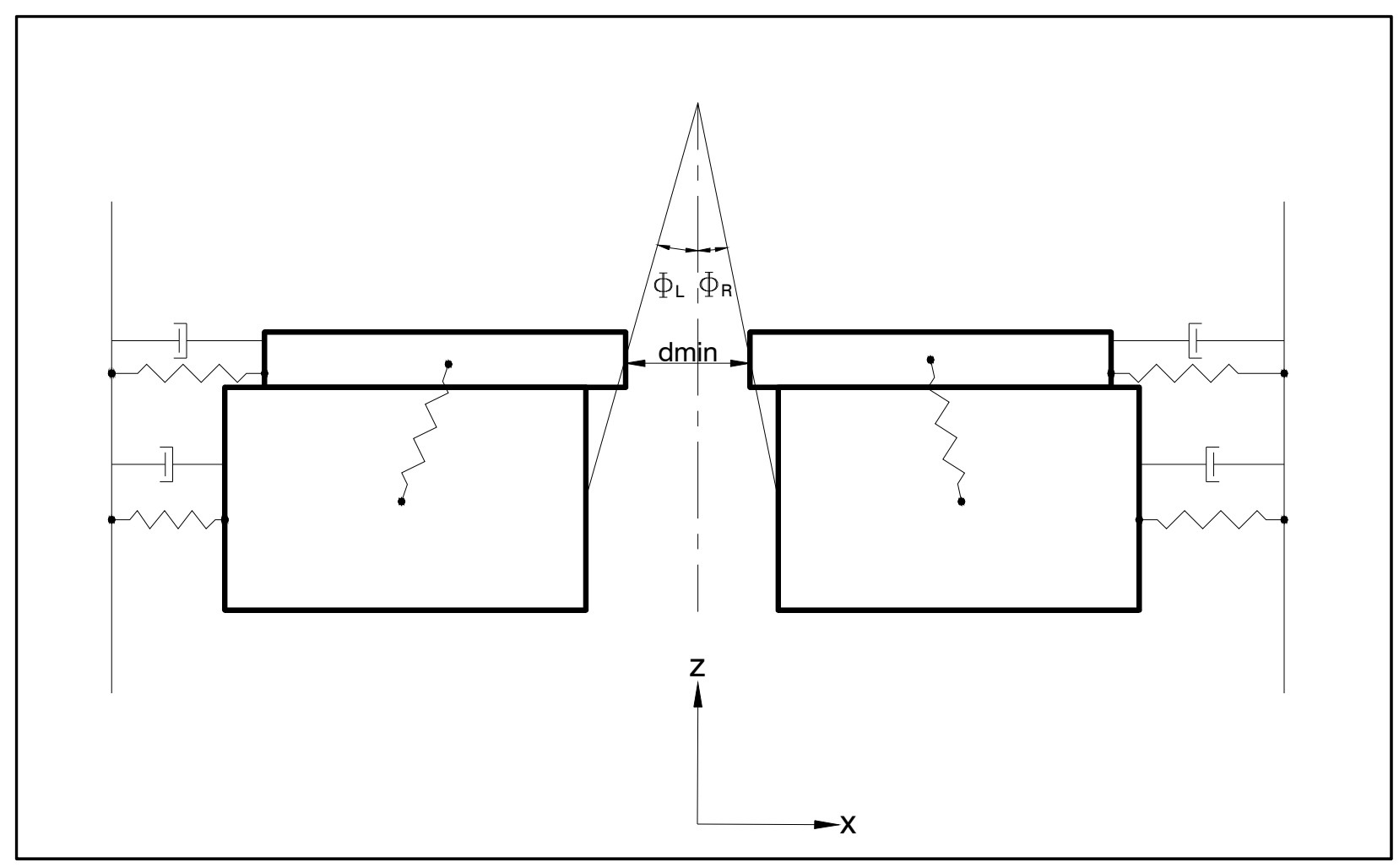

Figure 3-6 Identification of M5 variables in the asymmetric two-mass model

Organization of the data in this way converted the static M5 data into a dynamic function of time that was used to calculate the driving forces for the asymmetric two-mass model. However, since the range of the M5 data function was limited, a modified version of the IF72 forces was used to supplement the M5 data. A logical operator was applied in the Mathematica program to switch between the M5 pressure data and the modified IF72 forces, as appropriate.

In the IF72 model, the pressure on the medial surfaces of the vocal folds is inversely related to the glottal area. The equations are provided in Chapter 2 but are repeated here for reference.

$$
\begin{aligned}
& P_{m 1}=P_{s}-1.37 \frac{\rho}{2}\left(\frac{U_{g}}{A_{g 1}}\right)^{2}-\frac{1}{2}\left(\frac{12 \mu l_{g} d_{1}}{A_{g 1}^{3}} U_{g}+\frac{\rho d_{1}}{A_{g 1}} \frac{d U_{g}}{d t}\right) \\
& P_{m 2}=P_{m 1}-\frac{1}{2}\left[\left(\frac{12 \mu l_{g} d_{1}}{A_{g 1}^{3}}+\frac{12 \mu l_{g} d_{2}}{A_{g 2}^{3}}\right) U_{g}+\left(\frac{\rho d_{1}}{A_{g 1}}+\frac{\rho d_{2}}{A_{g 2}}\right) \frac{d U_{g}}{d t}\right]-\frac{1}{2} \rho U_{g}^{2}\left(\frac{1}{A_{g 2}^{2}}-\frac{1}{A_{g 1}^{2}}\right) .
\end{aligned}
$$


These inverse relations to the area result in unrealistically large, inward forces during the closing, or divergent, part of the cycle as the area approaches zero. A consistent feature identified in the M5 data was that during the divergent part of the cycle the intraglottal pressure was typically a negative value of magnitude between $0 \%$ and $20 \%$ of the subglottal pressure. This observation was used as a basis to modify the IF72 forces by truncating the forces during the divergent part of the cycle to $-20 \%$ of the subglottal pressure. It is instructive to compare the forces during a few complete cycles of oscillation. Figure 3-7 provides this comparison for the original IF72 force, the modified IF72 force, and the M5 force on mass $m_{R 1}$. All forces were calculated based on the "typical" IF72 parameters presented in Chapter 2 and those to be discussed in Chapter 4. The rectangles in Figure 3-7(c) indicate the configuration of the vocal folds at particular times during the cycle.

It is evident from Figure 3-7 that the inward force during the divergent part of the cycle for the original IF72 model (upper graph) is much greater than those observed experimentally. The modified IF72 force is slightly realistic (center graph). The M5 force (lower graph) is based on static experimental data which were converted into a dynamic form.

In consideration of the above discussion, the logical conditions which govern the application of the forces applied in this study are necessarily more complicated than those of the IF72 model alone, as given in Table 2-2. Tables 3-1 and 3-2 provide the force conditions for the model applied in this study, depending on whether the modified IF72 forces (Table 3-1) or the M5 data (Table 3-2) are used. 

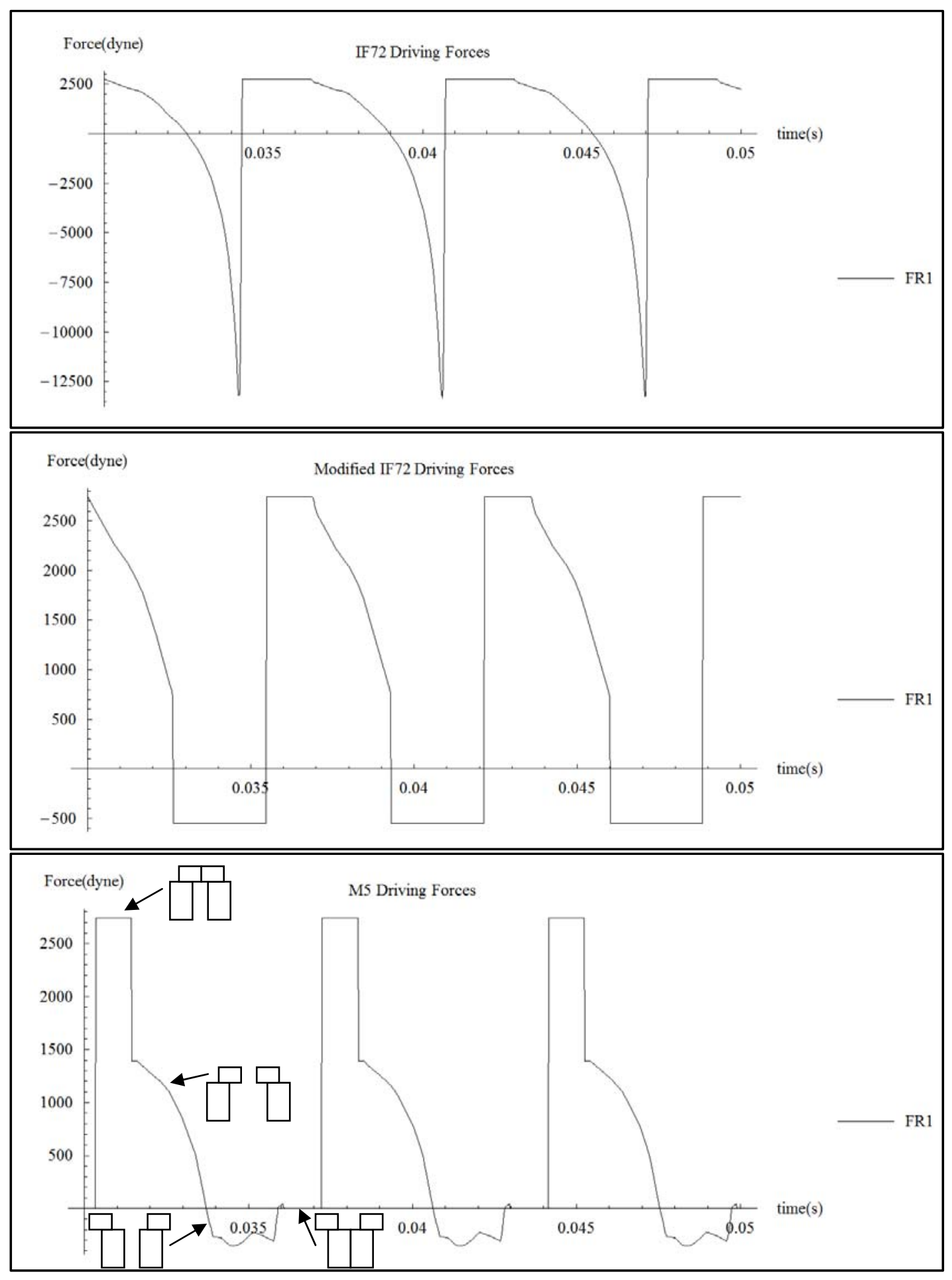

Figure 3-7 Driving force on mass $m_{R 1}$ for the typical IF72 model (upper), the modified IF72 force (center), and the M5 experimental data (lower). The transglottal pressure is $8 \mathrm{~cm} \mathrm{H}_{2} 0$ and the glottal area is $0.05 \mathrm{~cm}^{2}$. 
Table 3-1 Force conditions if the modified IF72 model pressures are applied

\begin{tabular}{|c|c|c|c|c|c|}
\hline$A_{g 1}$ & $\mathbf{A}_{\mathrm{g} 2}$ & $F_{R 1}$ & $F_{R 2}$ & $F_{L 1}$ & $\mathbf{F}_{\mathrm{L} 2}$ \\
\hline $\mathrm{A}_{\mathrm{g} 1}<\mathrm{A}_{\mathrm{g} 2}$ & $\mathrm{~A}_{\mathrm{g} 2}>\mathrm{A}_{\mathrm{g} 1}$ & $-0.20 \mathrm{P}_{\mathrm{s}} \lg _{\mathrm{g}} \mathrm{d}_{\mathrm{R} 1}$ & 0 & $-0.20 P_{\mathrm{s}} \lg _{\mathrm{g}} \mathrm{d}_{\mathrm{L} 1}$ & 0 \\
\hline$A_{g 1}>A_{g 2}$ & $\mathrm{~A}_{\mathrm{g} 2}<\mathrm{A}_{\mathrm{g} 1}$ & $\mathrm{P}_{\mathrm{m} 1} 1_{\mathrm{g}} \mathrm{d}_{\mathrm{R} 1}$ & $\mathrm{P}_{\mathrm{m} 2} 1_{\mathrm{g}} \mathrm{d}_{\mathrm{R} 2}$ & $P_{\mathrm{m} 1} l_{\mathrm{g}} \mathrm{d}_{\mathrm{L}}$ & $P_{\mathrm{m} 2} l_{\mathrm{g}} d_{\mathrm{L} 2}$ \\
\hline $\mathrm{A}_{\mathrm{g} 1}<0$ & $\mathrm{~A}_{\mathrm{g} 2}>0$ & $P_{\mathrm{S}} 1_{\mathrm{g}} \mathrm{d}_{\mathrm{R} 1}$ & 0 & $P_{\mathrm{S}} 1_{\mathrm{g}} d_{\mathrm{L} 1}$ & 0 \\
\hline $\mathrm{A}_{\mathrm{g} 1}<0$ & $\mathrm{~A}_{\mathrm{g} 2}<0$ & $P_{S} l_{g} d_{R 1}$ & 0 & $P_{S} l_{g} d_{L 1}$ & 0 \\
\hline $\mathrm{A}_{\mathrm{g} 1}>0$ & $\mathrm{~A}_{\mathrm{g} 2}<0$ & $\mathrm{P}_{\mathrm{s}} \mathrm{l}_{\mathrm{g}} \mathrm{d}_{\mathrm{R} 1}$ & $\mathrm{P}_{\mathrm{S}} \mathrm{l}_{\mathrm{g}} \mathrm{d}_{\mathrm{R} 2}$ & $\mathrm{P}_{\mathrm{s}} \mathrm{l}_{\mathrm{g}} \mathrm{d}_{\mathrm{L} 1}$ & $\mathrm{P}_{\mathrm{S}} \lg _{\mathrm{g}} \mathrm{d}_{\mathrm{L} 2}$ \\
\hline
\end{tabular}

Table 3-2 Force conditions if the M5 pressure data is applied

\begin{tabular}{|c|c|c|c|c|c|}
\hline $\mathbf{A}_{\mathrm{g} 1}$ & $\mathbf{A}_{\mathrm{g} 2}$ & $F_{R 1}$ & $F_{R 2}$ & $F_{L 1}$ & $\mathbf{F}_{\mathbf{L} 2}$ \\
\hline$A_{g 1}>0$ & $\mathrm{~A}_{\mathrm{g} 2}>0$ & $\begin{array}{l}\left\langle\mathrm{P}_{\mathrm{M} 5 \mathrm{NFW} 1}\right\rangle \mathrm{l}_{\mathrm{g}} \\
\mathrm{d}_{\mathrm{R} 1}\end{array}$ & $\begin{array}{l}\left\langle\mathrm{P}_{\mathrm{M} 5 \mathrm{NFW} 2}\right\rangle \mathrm{l}_{\mathrm{g}} \\
\mathrm{d}_{\mathrm{R} 2}\end{array}$ & $\begin{array}{l}\left\langle\mathrm{P}_{\mathrm{M} 5 \mathrm{FW} 1}\right\rangle \mathrm{l}_{\mathrm{g}} \\
\mathrm{d}_{\mathrm{L} 1}\end{array}$ & $\begin{array}{l}\left\langle\mathrm{P}_{\mathrm{M} 5 \mathrm{NFW} 2}\right\rangle \mathrm{l}_{\mathrm{g}} \\
\mathrm{d}_{\mathrm{L} 2}\end{array}$ \\
\hline $\mathrm{A}_{\mathrm{g} 1}>0$ & $\mathrm{~A}_{\mathrm{g} 2}<0$ & $\mathrm{P}_{\mathrm{s}} 1_{\mathrm{g}} \mathrm{d}_{\mathrm{R} 1}$ & 0 & $\mathrm{P}_{\mathrm{s}} 1_{\mathrm{g}} \mathrm{d}_{\mathrm{L} 1}$ & 0 \\
\hline $\mathrm{A}_{\mathrm{g} 1}<0$ & $\begin{array}{c}\mathrm{A}_{\mathrm{g} 2}<0 \text { or } \\
\mathrm{A}_{\mathrm{g} 2}>0\end{array}$ & 0 & 0 & 0 & 0 \\
\hline
\end{tabular}

Note: $\left\langle\mathrm{P}_{\mathrm{M} 5 \mathrm{NFW} 1}\right\rangle,\left\langle\mathrm{P}_{\mathrm{M} 5 \mathrm{NFW} 2}\right\rangle,\left\langle\mathrm{P}_{\mathrm{M} 5 \mathrm{FW} 1}\right\rangle$, and $\left\langle\mathrm{P}_{\mathrm{M} 5 \mathrm{NFW} 2}\right\rangle$ are the average values of the $\mathrm{M} 5$ pressure function at the appropriate locations. The right vocal fold was considered the non-flow wall for typical conditions.

\subsection{The Vocal Tract}

The feedback mechanism between the vocal folds and the vocal tract is not incorporated into this model. However, the calculated glottal airflow rate is inserted into the vocal tract flow equations to determine the general characteristics of the output sound. The vocal tract model applied in this study is the same as the network method in the IF72 model with the first-order differential equations (2.11) converted to second-order equations for the sake of computational uniformity. The equations employed in this model are given below.

$$
\begin{aligned}
& \left(R_{k 1}+R_{k 2}\right)\left|\dot{q}_{g}\right| \dot{q}_{g}+\left(R_{v 1}+R_{v 2}\right) \dot{q}_{g}+\left(L_{g 1}+L_{g 2}\right) \ddot{q}_{g}+L_{1} \ddot{q}_{g}+R_{1} \dot{q}_{g}+ \\
& \quad \frac{1}{C_{1}}\left(q_{g}-q_{1}\right)-P_{s}=0, \\
& \left(L_{1}+L_{2}\right) \ddot{q}_{1}+\left(R_{1}+R_{2}\right) \dot{q}_{1}+\frac{1}{C_{2}}\left(q_{1}-q_{2}\right)+\frac{1}{C_{1}}\left(q_{1}-q_{g}\right)=0, \\
& \left(L_{2}+L_{3}\right) \ddot{q}_{2}+\left(R_{2}+R_{3}\right) \dot{q}_{2}+\frac{1}{C_{3}}\left(q_{2}-q_{3}\right)+\frac{1}{C_{2}}\left(q_{2}-q_{1}\right)=0,
\end{aligned}
$$




$$
\begin{aligned}
& \left(L_{3}+L_{4}\right) \ddot{q}_{3}+\left(R_{3}+R_{4}\right) \dot{q}_{3}+\frac{1}{c_{4}}\left(q_{3}-q_{4}\right)+\frac{1}{c_{3}}\left(q_{3}-q_{2}\right)=0, \\
& \left(L_{4}+L_{r}\right) \ddot{q}_{4}+R_{4} \dot{q}_{4}-L_{r} \ddot{q}_{r}+\frac{1}{c_{4}}\left(q_{4}-q_{3}\right)=0, \\
& L_{r}\left(\ddot{q}_{r}-\ddot{q}_{4}\right)+R_{r} \dot{q}_{r}=0 .
\end{aligned}
$$

The terms $q_{g}, q_{1}, q_{2}, q_{3}, q_{4}$, and $q_{5}$ represent volumes of air, which are the equivalent of charge in the electrical circuit analogue, for the glottal area and each of the vocal tract segments downstream of the glottis, respectively. Conversion of the first-order differential equations (2.11) to the second-order form of equations (3.4) assumed that the initial volumes of air were all set to zero. The $\dot{q}$ terms are the first derivative of the air volumes with respect to time which provide the volumetric flow rate of air, denoted earlier by $U_{i}$. The second derivatives of the air volumes with respect to time, $\ddot{q}$, provide the acceleration of the airflow, denoted earlier by $d U_{i} / d t$. The remaining inductance, resistance, and capacitance terms in equations (3.4) are defined in Chapter 2.

\subsection{Model Pros and Cons}

At this point it is useful to summarize the advantages and disadvantages of the model applied in this study. The advantages are:

1) The computational efficiency of the model allows for numerous runs to be calculated with almost instant results. This facilitates changes of parameters without much effort, which allows the user to explore many different possibilities.

2) The incorporation of the M5 pressure data in the model provides a means of analyzing the effects of real driving forces. This may be the best quasi-static method for including the driving forces in a lumped element model since it automatically handles jet formation and properly distinguishes between convergent and divergent glottal configurations. 
3) The minimal degrees of freedom reduce the number of tunable parameters. As such, one can more readily connect an observed effect to the underlying cause. This is not a simple task in the more complex models.

4) Asymmetries and random fluctuations can be added at various points. This facilitates the investigation of a broad spectrum of questions about small changes in the parameters and forces.

The disadvantages are:

1) The lumped element approach inherently results in the "washing out" of the fine details of the real system. Specifically, pressure distributions must be averaged over relatively large areas and the limited number of degrees of freedom may be too coarse to allow the expression of some important aspects of the aerodynamics and oscillator motion.

2) The experimental data used in this study are for the case of symmetric vocal fold configurations only. One should think of this as a starting point for this type of model until more asymmetric pressure data become available. Nevertheless, it will be shown that when the forces are manually adjusted to be asymmetric, the vocal folds tend to entrain, thereby limiting the effects of the asymmetry. This result suggests that asymmetric driving forces may not have a significant effect on vocal fold dynamics.

3) Another limitation of the use of experimental data in this study is that it is only applicable to cases where the sizes of the left and right vocal folds are the same. Therefore, it was not possible to test for different size vocal folds that may result from swelling or growths, such as polyps or tumors.

The lumped element model employed in this study is true to the natural system from the perspective that the inputs to the model are only the subglottal pressure, the geometry of the 
system (which is time-dependent), and the biomechanical parameters. Given these inputs and the relations defined by the model, the generation of the glottal airflow is allowed to unfold in a natural way. Future studies will incorporate the feedback mechanism between the vocal tract and vocal folds to improve the comprehensiveness of the model. The methodology for this study will be discussed in the following chapter. 


\section{CHAPTER 4. METHODOLOGY}

The equations of motion given in equations (3.1), the vocal tract equations (3.5), and the force conditions given in Tables 3-1 and 3-2 comprise the mathematical model employed in this study. The system of ten, second order, ordinary differential equations was solved using the NDSolve function in Mathematica version 5.0 (32 bit). NDSolve is a general numerical differential equation solver that applies an adaptive algorithm to determine the appropriate step size during computation, minimizing the step size to track the solution to within the specified accuracy. The algorithm has several built in methods for calculating the solutions. If a method is not specified by the user, the most appropriate method or combination of methods is applied depending on the structure of the equations to be solved. In this study, Mathematica's adaptive algorithm was allowed to select the method. NDSolve is composed of approximately 1400 pages of Mathematica code. Most of the computations were conducted on a notebook computer with an Advanced Micro Devices (AMD) Turion $64 \mathrm{X} 2$ processor. The ratio of computation time to real life time was approximately 1000:1. Most of the model runs were conducted for a total time of 500 milliseconds, which required approximately 500 seconds (8.33 minutes) of computation time. Most of the computation time was required to handle the M5 data and the many logical conditions incorporated in the model.

The solutions of the equations produced by the NDSolve algorithm are numerical interpolating functions that can be treated like any other mathematical function. For example, one can perform the methods of calculus, statistics, and graphical analysis to study the solutions. In this case, the ten coupled differential equations of the model were solved by obtaining ten solutions in the form of interpolating functions of time. The solution set included the 
displacement equations of the four oscillators and the six volumetric airflow equations for the glottis, the four segments of the vocal tract, and the mouth output segment.

The general procedure for testing the hypotheses of this study entailed the following steps:

1) Solving the system of equations with the "typical" IF72 model conditions. This solution formed the control system for the simulations.

2) Each hypothesis concerning the perturbations (pages 6-7 above) was tested by systematically varying appropriate input parameters or initial conditions by different amounts, then solving the equations with the new inputs.

3) The solutions of the model in each case were presented graphically to observe the time dependence of the oscillator displacements, the glottal areas, the glottal airflow, mouth output sound pressure, and the driving forces on the oscillators.

4) Jitter and shimmer plots were created to identify evidence of fundamental frequency or amplitude perturbations in the glottal airflow.

5) Numerical jitter and shimmer measures were calculated to quantify the overall perturbation level in the fundamental frequency and amplitude.

The model was initially solved with the typical IF72 parameters to ensure that reasonable results could be obtained. These typical parameters were then varied to test the four hypotheses of this study. In each case, an attempt was made to determine the physiological significance of the modification. It was necessary to expand the set of "typical" IF72 parameters to allow for bilateral asymmetry. The full suite of typical parameters applied in this model is provided in Table 4-1. With respect to the driving forces for the typical case, the right vocal fold was maintained as the non-flow and the left vocal fold was the flow wall. For the purposes of this study, the interaction between the glottal airflow and the vocal tract was not considered. 
However, the glottal airflow was inserted into the flow equations to determine general output sound characteristics.

Table 4-1 List of parameters and their values for the asymmetric two-mass model

\begin{tabular}{|c|c|c|}
\hline Parameter & Description & Typical Value \\
\hline $\mathrm{m}_{\mathrm{R} 1}$ & mass of lower right oscillator & $0.125 \mathrm{~g}$ \\
\hline $\mathrm{m}_{\mathrm{R} 2}$ & mass of upper right oscillator & $0.025 \mathrm{~g}$ \\
\hline $\mathrm{m}_{\mathrm{L} 1}$ & mass of lower left oscillator & $0.125 \mathrm{~g}$ \\
\hline $\mathrm{m}_{\mathrm{L} 2}$ & mass of upper left oscillator & $0.025 \mathrm{~g}$ \\
\hline $\mathrm{d}_{\mathrm{g} 1}$ & thickness of lower mass (inferior/superior) & $0.25 \mathrm{~cm}$ \\
\hline $\mathrm{d}_{\mathrm{g} 2}$ & thickness of upper mass (inferior/superior) & $0.05 \mathrm{~cm}$ \\
\hline $1 \mathrm{~g}$ & length of vocal folds (anterior/posterior) & $1.4 \mathrm{~cm}$ \\
\hline $\mathrm{k}_{\mathrm{R} 1}$ & spring constant, lower right mass, open glottis & 80,000 dyne/cm \\
\hline $\mathrm{k}_{\mathrm{R} 2}$ & spring constant, upper right mass, open glottis & 8,000 dyne/cm \\
\hline $\mathrm{k}_{\mathrm{L} 1}$ & spring constant, lower left mass, open glottis & 80,000 dyne/cm \\
\hline $\mathrm{k}_{\mathrm{L} 2}$ & spring constant, upper left mass, open glottis & 8,000 dyne/cm \\
\hline $\mathrm{h}_{\mathrm{R} 1}$ & spring constant, lower right mass, closed glottis & 240,000 dyne $/ \mathrm{cm}$ \\
\hline $\mathrm{h}_{\mathrm{R} 2}$ & spring constant, upper right mass, closed glottis & 24,000 dyne/cm \\
\hline $\mathrm{h}_{\mathrm{L} 1}$ & spring constant, lower left mass, closed glottis & 240,000 dyne $/ \mathrm{cm}$ \\
\hline $\mathrm{h}_{\mathrm{L} 2}$ & spring constant, upper left mass, closed glottis & 24,000 dyne/cm \\
\hline $\mathrm{k}_{\mathrm{Rc}}$ & coupling spring constant, right side & 25,000 dyne/cm \\
\hline $\mathrm{k}_{\mathrm{Lc}}$ & coupling spring constant, left side & 25,000 dyne/cm \\
\hline$\eta_{\mathrm{kR} 1}$ & nonlinear spring coefficient, upper right mass, open glottis & 100 \\
\hline$\eta_{\mathrm{kR} 2}$ & nonlinear spring coefficient, lower right mass, open glottis & 100 \\
\hline$\eta_{\mathrm{kL} 1}$ & nonlinear spring coefficient, upper left mass, open glottis & 100 \\
\hline$\eta_{\mathrm{kL} 2}$ & nonlinear spring coefficient, upper left mass, open glottis & 100 \\
\hline$\eta_{\mathrm{hR} 1}$ & $\begin{array}{l}\text { nonlinear spring coefficient, upper right mass, closed } \\
\text { glottis }\end{array}$ & 500 \\
\hline$\eta_{\mathrm{hR} 2}$ & $\begin{array}{l}\text { nonlinear spring coefficient, lower right mass, closed } \\
\text { glottis }\end{array}$ & 500 \\
\hline$\eta_{\mathrm{hL} 1}$ & nonlinear spring coefficient, upper left mass, closed glottis & 500 \\
\hline$\eta_{\mathrm{hL} 2}$ & nonlinear spring coefficient, upper left mass, closed glottis & 500 \\
\hline $\mathrm{r}_{\mathrm{R} 1}$ & viscous damping constant, lower right mass, open glottis & 20 dyne $\mathrm{s} / \mathrm{cm}$ \\
\hline $\mathrm{r}_{\mathrm{R} 2}$ & viscous damping constant, upper right mass, open glottis & 17 dyne s/cm \\
\hline $\mathrm{r}_{\mathrm{L} 1}$ & viscous damping constant, lower left mass, open glottis & 20 dyne $\mathrm{s} / \mathrm{cm}$ \\
\hline
\end{tabular}




\begin{tabular}{|c|c|c|}
\hline Parameter & Description & Typical Value \\
\hline $\mathrm{r}_{\mathrm{L} 2}$ & viscous damping constant, upper left mass, open glottis & 17 dyne s/cm \\
\hline $\mathrm{r}_{\mathrm{R} 1 \mathrm{c}}$ & viscous damping constant, lower right mass, closed glottis & 220 dyne $\mathrm{s} / \mathrm{cm}$ \\
\hline $\mathrm{r}_{\mathrm{R} 2 \mathrm{c}}$ & viscous damping constant, upper right mass, closed glottis & 45 dyne s/cm \\
\hline $\mathrm{r}_{\mathrm{L} 1 \mathrm{c}}$ & viscous damping constant, lower left mass, closed glottis & 220 dyne $\mathrm{s} / \mathrm{cm}$ \\
\hline $\mathrm{r}_{\mathrm{L} 2 \mathrm{c}}$ & viscous damping constant, upper left mass, closed glottis & 45 dyne $\mathrm{s} / \mathrm{cm}$ \\
\hline$\rho$ & density of warm, moist air & $1.14 \times 10^{-3} \mathrm{~g} / \mathrm{cm}^{3}$ \\
\hline$\mu$ & shear viscosity of air & $\begin{array}{l}1.8 \times 10^{-4} \text { dyne } \\
\mathrm{s} / \mathrm{cm}^{2}\end{array}$ \\
\hline $\mathrm{c}$ & speed of sound in warm air & $35,000 \mathrm{~cm} / \mathrm{s}$ \\
\hline$l_{\mathrm{i}}$ & length of cylindrical vocal tract segment, $\mathrm{i}=1,2,3,4$ & $4.25 \mathrm{~cm}$ \\
\hline $\mathrm{A}_{\mathrm{i}}$ & $\begin{array}{l}\text { area of cylindrical vocal tract segment, } \mathrm{i}=1,2,3,4,5 \\
\text { (including mouth area) }\end{array}$ & $5 \mathrm{~cm}^{2}$ \\
\hline $\mathrm{P}_{\mathrm{s}}$ & subglottal pressure & 7,840 dyne $/ \mathrm{cm}^{2}$ \\
\hline $\mathrm{A}_{\mathrm{g} 0 \mathrm{R} 1}$ & prephonatory (initial) glottal area, lower right side & $0.025 \mathrm{~cm}^{2}$ \\
\hline $\mathrm{A}_{\mathrm{g} 0 \mathrm{R} 2}$ & prephonatory (initial) glottal area, upper right side & $0.025 \mathrm{~cm}^{2}$ \\
\hline $\mathrm{A}_{\mathrm{g} 0 \mathrm{~L} 1}$ & prephonatory (initial) glottal area, lower left side & $0.025 \mathrm{~cm}^{2}$ \\
\hline $\mathrm{A}_{\mathrm{g} 0 \mathrm{~L} 2}$ & prephonatory (initial) glottal area, upper left side & $0.025 \mathrm{~cm}^{2}$ \\
\hline
\end{tabular}

4.1 Testing Hypothesis \#1: Time-independent Physical Asymmetry

The effects of time-independent physical asymmetry on the cycle-to-cycle period and amplitude of the glottal airflow was investigated by modifying specific model parameters. The parameters, modifications, and possible physiological connections are given in Table 4-2.

Table 4-2 Parameter modifications for testing hypothesis \#1

\begin{tabular}{|c|c|c|}
\hline Parameter & Modification & Physiological Significance \\
\hline $\mathrm{m}_{\mathrm{R} 1}$ & $\begin{array}{l}\text { increase by factors of } 1.05,1.10,1.20 \text {, } \\
1.50 \text {, and } 2.00\end{array}$ & $\begin{array}{l}\text { - vocal fold of greater mass on } \\
\text { lower, right side } \\
\text { - } \begin{array}{l}\text { greater tissue density on right } \\
\text { side }\end{array}\end{array}$ \\
\hline $\mathrm{m}_{\mathrm{R} 2}$ & $\begin{array}{l}\text { increase by factors of } 1.05,1.10,1.20 \text {, } \\
1.50 \text {, and } 2.00\end{array}$ & $\begin{array}{l}\text { vocal fold of greater mass on } \\
\text { upper, right side } \\
\text { - greater tissue density on right } \\
\text { side }\end{array}$ \\
\hline
\end{tabular}




\begin{tabular}{|c|c|c|}
\hline Parameter & Modification & Physiological Significance \\
\hline $\mathrm{k}_{\mathrm{R} 1}$ & $\begin{array}{l}\text { increase by factors of } 1.05,1.10,1.20 \text {, } \\
1.50 \text {, and } 2.00\end{array}$ & $\begin{array}{l}\text { - vocal fold of greater stiffness on } \\
\text { lower, right side } \\
\text { greater effective tension of } \\
\text { tissue in motion due to the } \\
\text { vocalis muscle }\end{array}$ \\
\hline $\mathrm{k}_{\mathrm{R} 2}$ & $\begin{array}{l}\text { increase by factors of } 1.05,1.10,1.12 \text {, } \\
1.15,1.20,1.50 \text {, and } 2.00\end{array}$ & $\begin{array}{l}\text { - vocal fold of greater stiffness on } \\
\text { upper, right side } \\
\text { - greater effective tension of } \\
\text { tissue in motion due to the } \\
\text { vocalis muscle }\end{array}$ \\
\hline $\mathrm{k}_{\mathrm{Rc}}$ & $\begin{array}{l}\text { increase by factors of } 1.05,1.10,1.18 \text {, } \\
1.20,1.22,1.50 \text {, and } 2.00\end{array}$ & $\begin{array}{l}\text { vocal fold cover of greater } \\
\text { stiffness on right side } \\
\text { greater effective tension of } \\
\text { tissue in motion due to the } \\
\text { cricothyroid muscle }\end{array}$ \\
\hline
\end{tabular}

\subsection{Testing Hypothesis \#2: Time-dependent Physical Asymmetry}

The effects of time-dependent physical asymmetry on the cycle-to-cycle period and amplitude of the glottal airflow was investigated by modifying specific model parameters. The parameters, modifications, and possible physiological connections are given in Table 4-3. All other parameters are as listed in Table 4-1.

Table 4-3 Parameter modifications for testing hypothesis \#2

\begin{tabular}{|c|c|c|}
\hline Parameter & Modification & Physiological Significance \\
\hline $\mathrm{k}_{\mathrm{R} 1}$ & $\begin{array}{l}\text { - } \mathrm{k}_{\mathrm{R} 1} \text { converted to a function of time, } \\
\mathrm{k}_{\mathrm{R} 1}(\mathrm{t}) \\
\text { the function varied the value of } \mathrm{k}_{\mathrm{R} 1} \\
\text { around the typical value by factors of } \\
1.005,1.01,1.05,1.10,1.25 \text { and } 1.50 \\
\text { variation implemented randomly (refer } \\
\text { to discussion below) }\end{array}$ & $\begin{array}{l}\text { - random variability in the } \\
\text { effective tension of the tissue in } \\
\text { motion due to the vocalis } \\
\text { muscle }\end{array}$ \\
\hline $\mathrm{k}_{\mathrm{R} 1}$ and $\mathrm{k}_{\mathrm{L} 1}$ & $\begin{array}{l}\text { - both } \mathrm{k}_{\mathrm{R} 1} \text { and } \mathrm{k}_{\mathrm{L} 1} \text { converted to functions } \\
\text { of time, } \mathrm{k}_{\mathrm{R} 1}(\mathrm{t}) \text { and } \mathrm{k}_{\mathrm{L} 1}(\mathrm{t}) \\
\text { - the function varied the typical values } \\
\text { by factors of } 1.005,1.01,1.05,1.10 \text {, }\end{array}$ & $\begin{array}{l}\text { - random variability of effective } \\
\text { tension of tissue in motion can } \\
\text { occur on both sides }\end{array}$ \\
\hline
\end{tabular}




\begin{tabular}{|c|c|c|}
\hline & $\begin{array}{ll} & 1.25 \text { and } 1.50 \\
- & \text { variation implemented randomly }\end{array}$ & \\
\hline $\begin{array}{l}\mathrm{k}_{\mathrm{R} 1}, \mathrm{k}_{\mathrm{R} 2}, \mathrm{k}_{\mathrm{L} 1}, \\
\mathrm{k}_{\mathrm{L} 2}, \mathrm{k}_{\mathrm{Rc}}, \mathrm{k}_{\mathrm{Lc}}\end{array}$ & $\begin{array}{l}\text { - all spring constants converted to } \\
\text { functions of time and varied randomly } \\
\text { around the typical values by factors of } \\
1.005,1.01,1.05,1.10,1.25 \text {, and } 1.50\end{array}$ & $\begin{array}{l}\text { - if random muscle tension } \\
\text { fluctuations are a real effect, } \\
\text { then all spring forces should be } \\
\text { subject to random variability }\end{array}$ \\
\hline
\end{tabular}

Several studies indicate that the tetanic contractions of the muscles that control the vocal folds are not constant in time (Alipour-Haghighi, Titze and Durham 1987; Alipour-Haghighi and Titze 1989; Titze 1991). Thus, the tensions of these muscles fluctuate while phonation is occurring, possibly resulting in jitter and shimmer. These fluctuations may be represented by time-varying spring constants in the asymmetric two-mass model. The nature of the tension perturbations applied in this study is based on work by Titze (1991) that models the thyroarytenoid muscle as a composition of 100 motor units. Each motor unit fires with a period based on a Gaussian distribution of random numbers with a mean of $40 \mathrm{~ms}$ and a standard deviation of $6 \mathrm{~ms}$. The amplitudes of the motor units vary with a uniform random distribution of various magnitudes. There is some evidence that the tension fluctuations are a primary reason for natural jitter and shimmer in the human voice (Baer 1978; Baer 1980). Other studies indicate that a less prominent cause of jitter is related to the heartbeat which has the effect of changing the tension in the vascular bed of the vocal folds in a more regular, nonrandom way (Orlikoff and Baken 1989a; Orlikoff and Baken 1989b). Since professional singers can be trained to minimize the amount of jitter and shimmer in their voices, it seems reasonable to postulate that if it is something within the realm of motor control then it is likely, at least in part, a neurological/motor control effect. The tension parameters in the asymmetric two-mass model have been varied individually and collectively to investigate these effects. 
Although the two-mass model has been criticized for an inability to represent the relative activation of separate muscle groups (Story and Titze 1995), this study is more concerned with overall effects related to physical and temporal asymmetries in the muscles rather than how these muscular asymmetries are connected with the relative activations of separate muscle groups.

\subsection{Testing Hypothesis \#3: Time-independent Asymmetric Driving Forces}

The effects of time-independent asymmetric driving forces on the cycle-to-cycle period and amplitude of the glottal airflow was investigated by modifying specific model parameters that described the driving forces. The parameters, modifications, and possible physiological connections are given in Table 4-4. All other parameters are as listed in Table 4-1.

Table 4-4 Parameter modifications for testing hypothesis \#3

\begin{tabular}{|c|c|c|}
\hline Parameter & Modification & Physiological Significance \\
\hline $\begin{array}{l}\mathrm{F}_{\mathrm{R} 1}, \mathrm{~F}_{\mathrm{R} 2} \\
\mathrm{~F}_{\mathrm{L} 1}, \text { and } \mathrm{F}_{\mathrm{L} 2}\end{array}$ & $\begin{array}{l}\text { allow the right side to be the flow wall and } \\
\text { the left to be the non-flow wall using the } \\
\text { M5 pressure data* }\end{array}$ & $\begin{array}{l}\text { - formation of an airflow jet } \\
\text { through the glottis } \\
\text { - in this case, the jet always forms } \\
\text { on the left side }\end{array}$ \\
\hline $\mathrm{F}_{\mathrm{R} 1}$ & $\begin{array}{l}\text { increase by factors of } 1.05,1.10,1.20 \text {, } \\
1.50 \text {, and } 2.00\end{array}$ & $\begin{array}{l}\text { - greater force on lower, right } \\
\text { vocal fold due to greater surface } \\
\text { area or unknown aerodynamic } \\
\text { causes }\end{array}$ \\
\hline $\mathrm{F}_{\mathrm{R} 2}$ & $\begin{array}{l}\text { increase by factors of } 1.05,1.10,1.20 \text {, } \\
1.50 \text {, and } 2.00\end{array}$ & $\begin{array}{l}\text { greater force on upper, right } \\
\text { vocal fold due to greater surface } \\
\text { area or unknown aerodynamic } \\
\text { causes }\end{array}$ \\
\hline
\end{tabular}

* This pertains to the typical case for this model and is, therefore, not actually a modification.

\subsection{Testing Hypothesis \#4: Time-dependent Asymmetric Driving Forces}

The effects of time-dependent asymmetric driving forces on the periodicity and amplitude of the glottal output was investigated by modifying specific model parameters. The parameters, modifications, and possible physiological connections are given in Table 4-5. 
Table 4-5 Parameter modifications for testing hypothesis \#4

\begin{tabular}{|c|c|c|}
\hline Parameter & Modification & Physiological Significance \\
\hline $\mathrm{F}_{\mathrm{R} 1}$ & $\begin{array}{l}\text { - random fluctuations of the force on the } \\
\text { lower mass of the right vocal fold } \\
\text { during the divergent part of the cycle } \\
\text { with magnitudes of }+/-1.01,1.05,1.10 \text {, } \\
1.25 \text {, and } 1.50 \\
\text { - period of variation was } 0.2 \mathrm{~ms}\end{array}$ & $\begin{array}{l}\text { - flow jet forms on the flow wall } \\
\text { resulting in pressure imbalances } \\
\text { between the flow wall and the } \\
\text { non-flow wall } \\
\text { this occurs on the lower portion } \\
\text { of the vocal fold during the } \\
\text { divergent part of the cycle }\end{array}$ \\
\hline $\mathrm{F}_{\mathrm{R} 1}$ and $\mathrm{F}_{\mathrm{R} 2}$ & $\begin{array}{l}\text { random fluctuations of the force on the } \\
\text { entire right vocal fold during the } \\
\text { divergent part of the cycle } \\
\text { random fluctuations of the force on } \\
\text { upper mass of the right vocal fold } \\
\text { during the whole cycle } \\
\text { - fluctuation magnitudes of }+/-1.01 \text {, } \\
1.05,1.10,1.25 \text {, and } 1.50 \\
\text { - period of variation was } 0.2 \mathrm{~ms}\end{array}$ & $\begin{array}{l}\text { - flow jet forms on the flow wall } \\
\text { resulting in pressure imbalances } \\
\text { between the flow wall and the } \\
\text { non-flow wall } \\
\text { this occurs during the divergent } \\
\text { part of the cycle on the lower } \\
\text { portion of the vocal fold and } \\
\text { during the entire cycle on the } \\
\text { upper portion of the vocal fold }\end{array}$ \\
\hline
\end{tabular}

It is well known that an air jet is formed in the glottis during the converging, rectangular, and diverging glottal shapes of phonation (Pelorson, Hirschberg, van Hassel et al. 1994; Scherer, Shinwari, De Witt et al. 2001; Scherer, Shinwari, De Witt et al. 2002; Thapa 2005). Along with jet formation, turbulent regions are generated that result in complex fluid mechanical behavior that is the subject of much current study (Neubauer, Zhang, Miraghaie et al. 2007). The jet flow results in the formation of a pressure imbalance and possibly unpredictable fluctuations in the forces that act on the vocal folds. It has been inferred that these fluctuating forces result in jitter and shimmer (Baken and Orlikoff 2000). As stated in the Introduction, one purpose of this study is to investigate the possible effects of these force fluctuations without having a complete knowledge of the complex fluid mechanical details. This is accomplished by adding varying degrees of randomness to the forces acting on one of the vocal fold segments. 


\subsection{Measures of Jitter and Shimmer}

In this study, the glottal flow pulse was used to calculate any cycle-to-cycle variations in the fundamental period or amplitude for the various scenarios presented above. Specifically, setting the first derivative of the glottal flow pulse with respect to time equal to zero, and solving for this time, provided the times at which the peak flows occurred. The magnitude of the positive peaks (amplitudes) where then determined at those times. The periods between those times were used to calculate the cycle-to-cycle period. The overall average amplitude and period were obtained from these data points. Jitter plots were then developed by graphing the period of each cycle normalized to the average period versus time. Shimmer plots were developed in a similar manner using the amplitude data. The glottal flow function was used for this analysis because it provided a smoother function to work with than the output sound pressure.

Numerical jitter and shimmer indices were also calculated to quantify the perturbations in a somewhat standard way. The indices applied here are based on the information contained in Baken and Orlikoff (2000). Jitter was quantified using the Jitter Factor (JF) of Hollien, Michel and Doherty (1973), which is given by

$$
J F=\frac{\frac{1}{n-1}\left[\sum_{i=1}^{n-1}\left|f_{i}-f_{i-1}\right|\right]}{\frac{1}{n} \sum_{i=1}^{n} f_{i}},
$$

where $f_{i}$ is the $\mathrm{i}^{\text {th }}$ frequency in the sample, and $n$ is the number of the samples. A typical JF for a normal voice is around $0.5 \%$, while for a disordered voice it may be as large as $3 \%$ to $5 \%$.

Shimmer was quantified using the amplitude variability index (AVI) of Deal and Emanuel (1978), which is related to the coefficient of variation (CV) defined as follows:

$$
\begin{aligned}
& C V=\frac{\frac{1}{n} \sum_{i=1}^{n}\left(x_{i}-\bar{x}\right)^{2}}{\bar{x}^{2}}, \\
& A V I=\log _{10}(C V \times 1000),
\end{aligned}
$$


where $\bar{x}$ is the average amplitude, $x_{i}$ is the $\mathrm{i}^{\text {th }}$ amplitude, and $n$ is the number of samples. The AVI for a normal voice may be on the order of -0.1 , while for a disordered voice an AVI of 0.1 to 0.4 may be expected. 


\section{CHAPTER 5. RESULTS AND DISCUSSION}

Prior to investigating the four hypotheses of this study, the asymmetric two-mass model was solved using the typical input parameters listed in Table 4-1 with a subglottal pressure of 8 $\mathrm{cm} \mathrm{H}_{2} \mathrm{O}\left(7840\right.$ dyne $\left./ \mathrm{cm}^{2}\right)$ and a uniform, prephonatory area of $0.05 \mathrm{~cm}^{2}$. The results for the oscillator displacements, glottal airflow rate, and output sound pressure are presented in Figure 5-1 (a), (b), and (c), respectively.

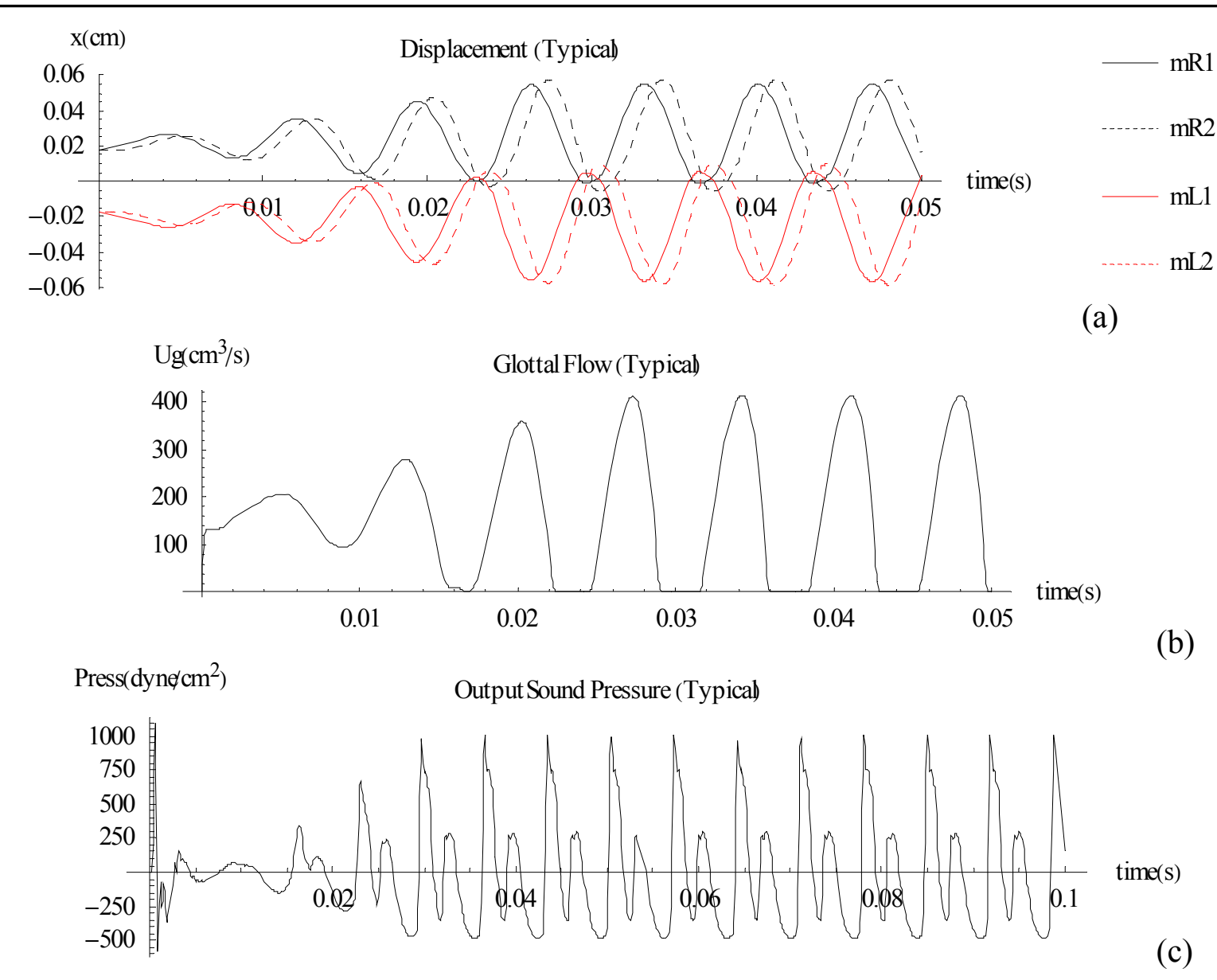

Figure 5-1 Results for the typical parameter set as given in Table 4-1 and applying the M5 pressure data to calculate the driving forces: (a) oscillator displacement; (b) glottal airflow rate; and (c) output sound pressure level.

The displacement graph of Figure 5-1(a) indicates a phase difference between the lower and upper masses of approximately 60 degrees, which is in accordance with the IF72 result of 55 
degrees. One difference between the results above and those of the IF72 model is that the on-set time in this model is longer owing to the more gentle forces given by the M5 pressure data. The on-set time here is approximately $28 \mathrm{~ms}$ compared to approximately $12 \mathrm{~ms}$ for the canonical IF72 model. An analysis of the glottal flow pulse over a time span of $450 \mathrm{~ms}$ indicates that no jitter or shimmer were detectable. This is expected for the perfectly symmetric case. However, the control case in this study is not perfectly symmetrical because the right vocal fold is always considered the flow wall and the left vocal fold is considered the non-flow wall. Therefore, although a bilateral pressure imbalance exists in the control case, jitter and shimmer are not detected. Close numerical examination of the control case results indicates that the amplitude of the right vocal is slightly greater than the left vocal fold. The fundamental period contour and the glottal airflow contour, both normalized to the respective averages, are provided in Figure 52.

\subsection{Hypothesis \#1: Time-independent Physical Asymmetry}

The effects of time-independent physical asymmetries were investigated by altering the masses and tensions of the right vocal fold according to the modifications detailed in Table 4-2. The following subsections present the results of the investigation.

\subsubsection{Asymmetry in the Lower, Right Mass}

The most instructive cases for this test are increases of the lower, right mass $\left(m_{R 1}\right)$ by factors of 50\% and 100\%. The results are presented in Figures 5-3 and 5-4. 

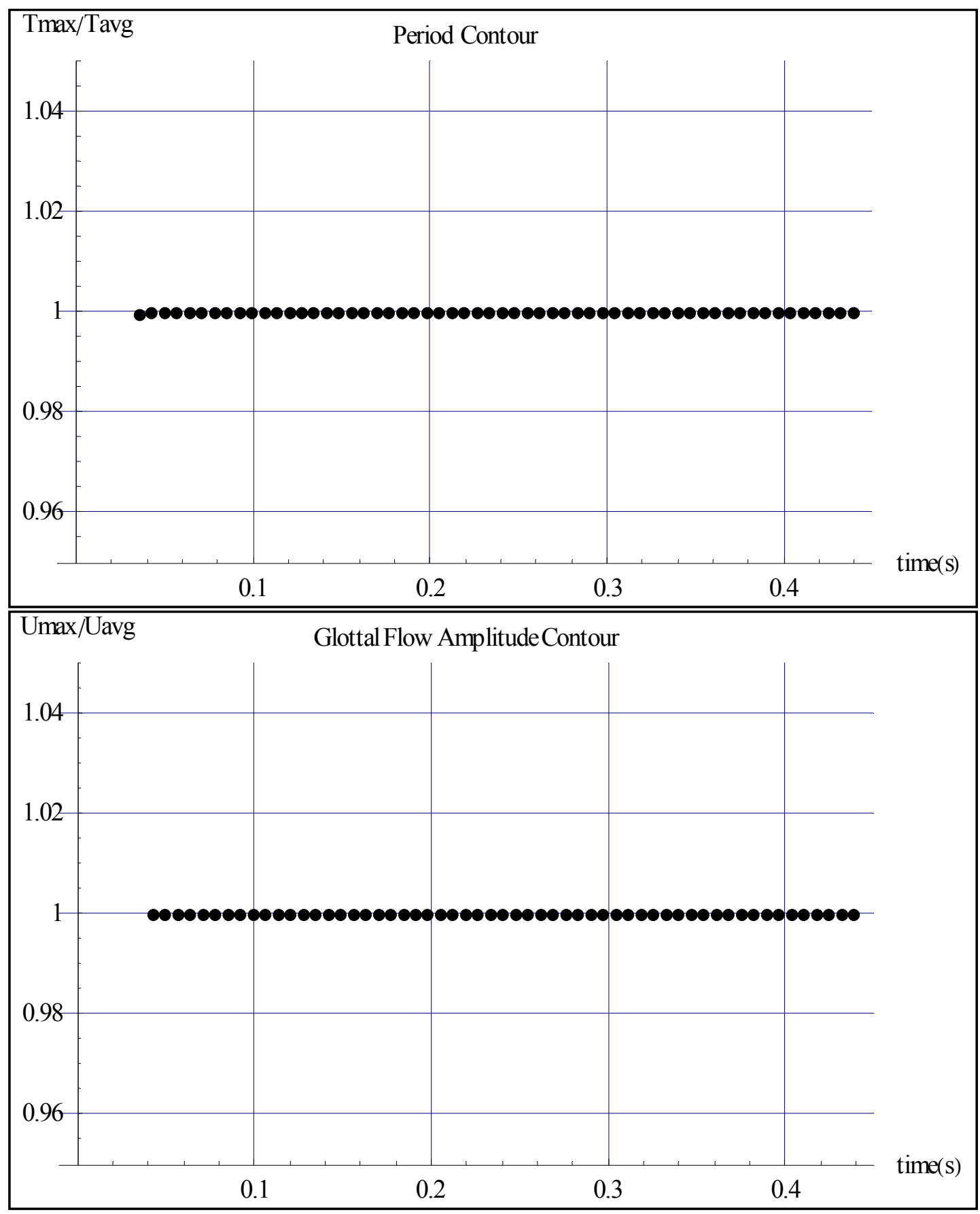

Figure 5-2 Fundamental period contour normalized to the average period and glottal airflow amplitude contour normalized to the average amplitude for the control case. 


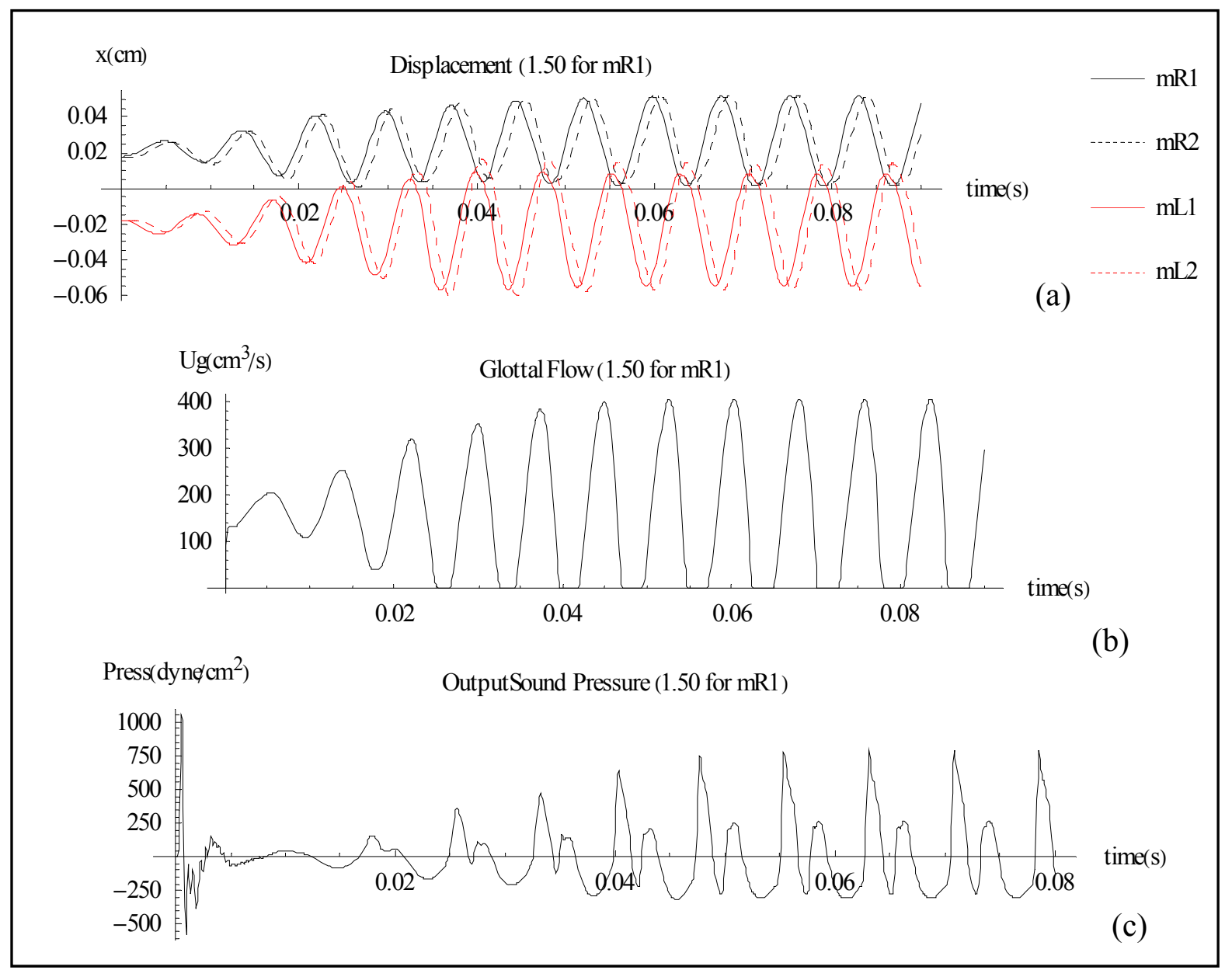

Figure 5-3 Results for an increase in the lower, right mass by a factor of 1.50: (a) oscillator displacements, (b) glottal flow, and (c) output sound pressure level 


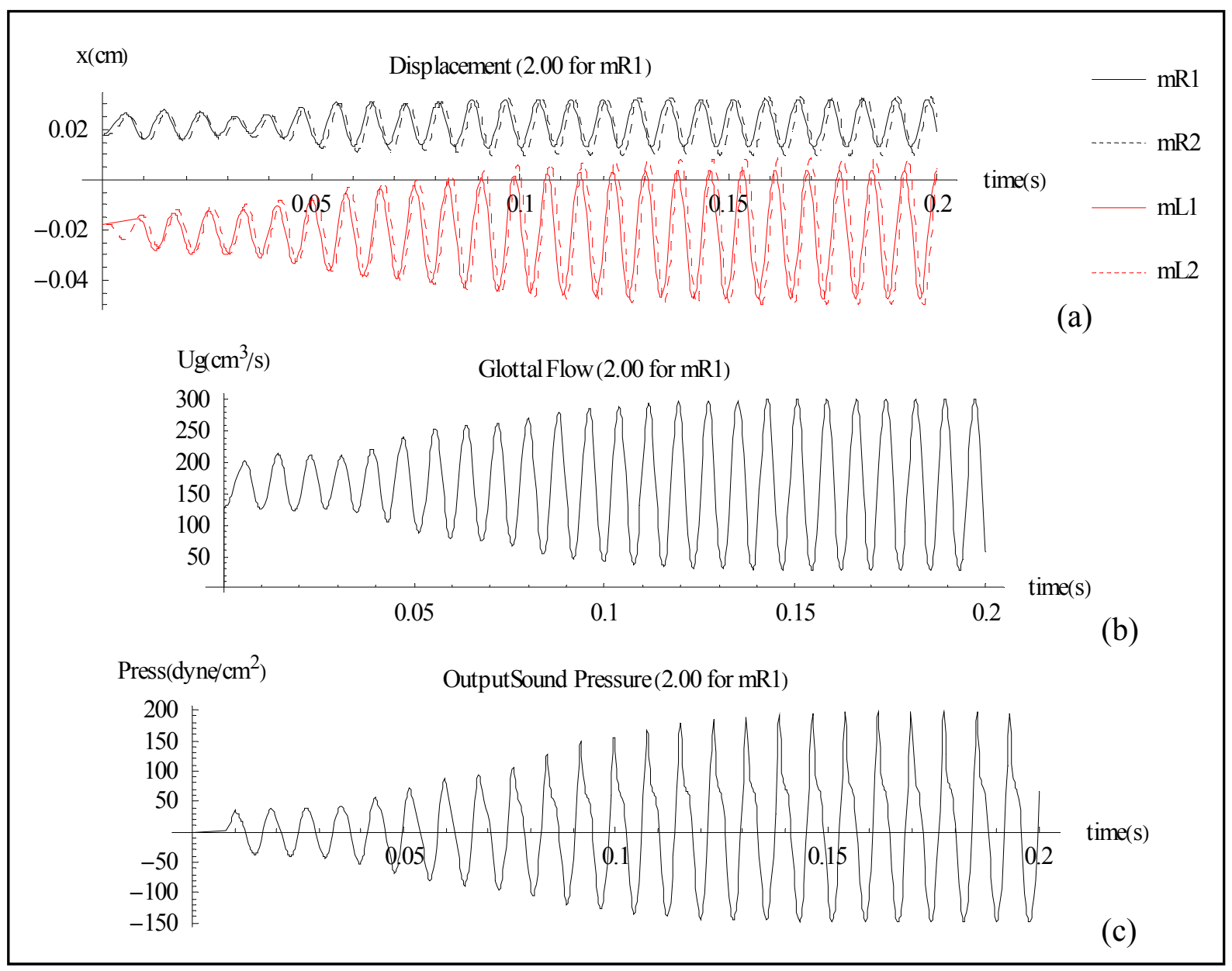

Figure 5-4 Results for an increase in the lower, right mass by a factor of 2.00: (a) oscillator displacements, (b) glottal flow, and (c) output sound pressure level.

As can be seen in the Figures 5-3(a) and 5-4(a), as the mass of the lower, right vocal fold is increased the amplitude of the right side decreases but the frequencies of the right and left vocal folds remain equal with only a relative phase difference between them. As the mass is increased by a factor of two, the right vocal fold becomes very sluggish resulting in a long on-set time, and the glottis does not completely close. Even in the extreme case of $100 \%$ mass increase on one side, the vocal folds entrain and settle on a common frequency. Regardless of the asymmetries in the oscillators, the model does not exhibit measureable jitter or shimmer even for the most extreme case, as shown in Figure 5-5. A graph of the fundamental frequency and the 
phase difference between the right and left vocal folds with respect to the multiplicative factor of $m_{R 1}$ is given in Figure 5-6.
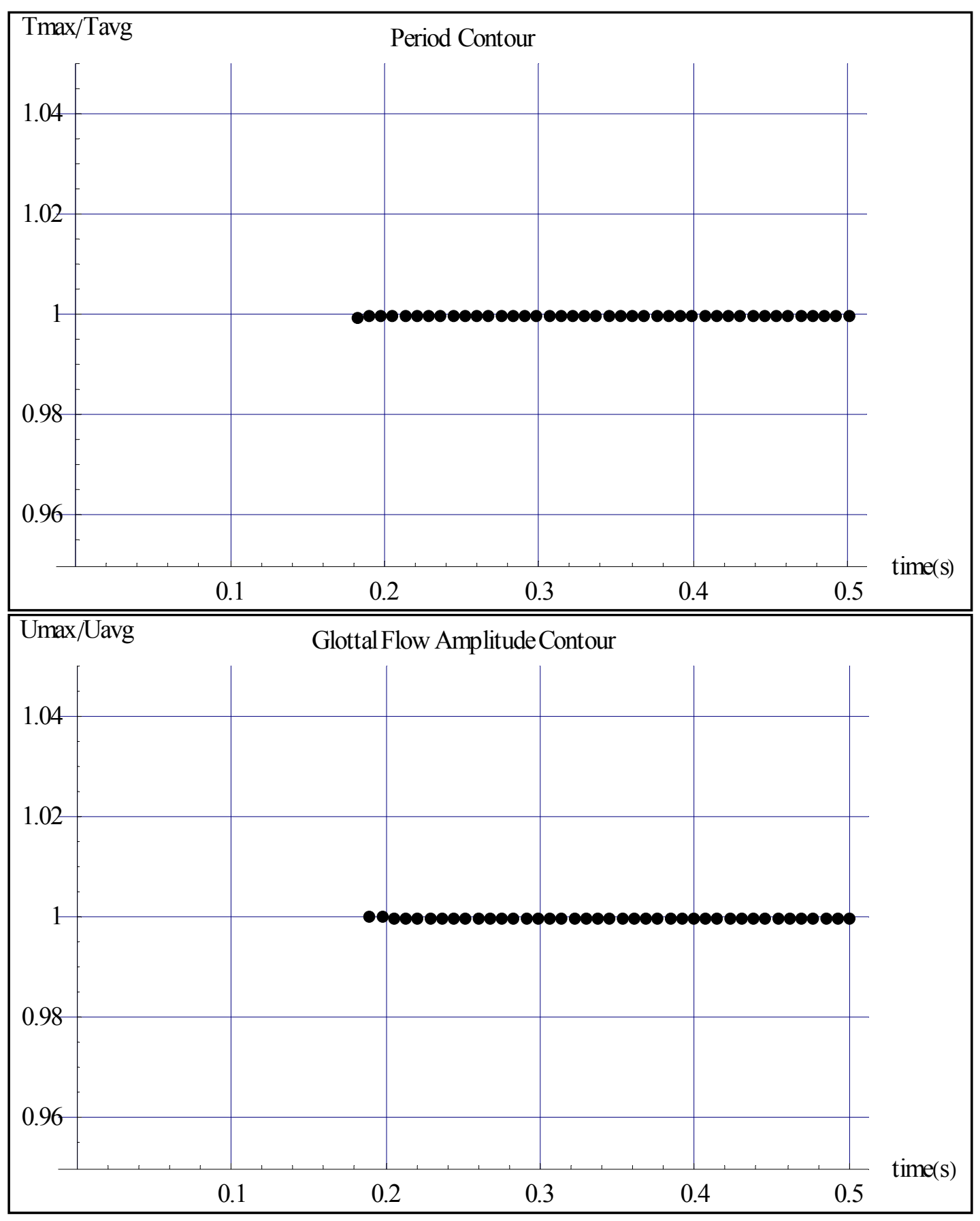

Figure 5-5 Fundamental period contour normalized to the average period and glottal airflow amplitude contour normalized to the average amplitude when $m_{R 1}$ is increased by a factor of 2.00 . 


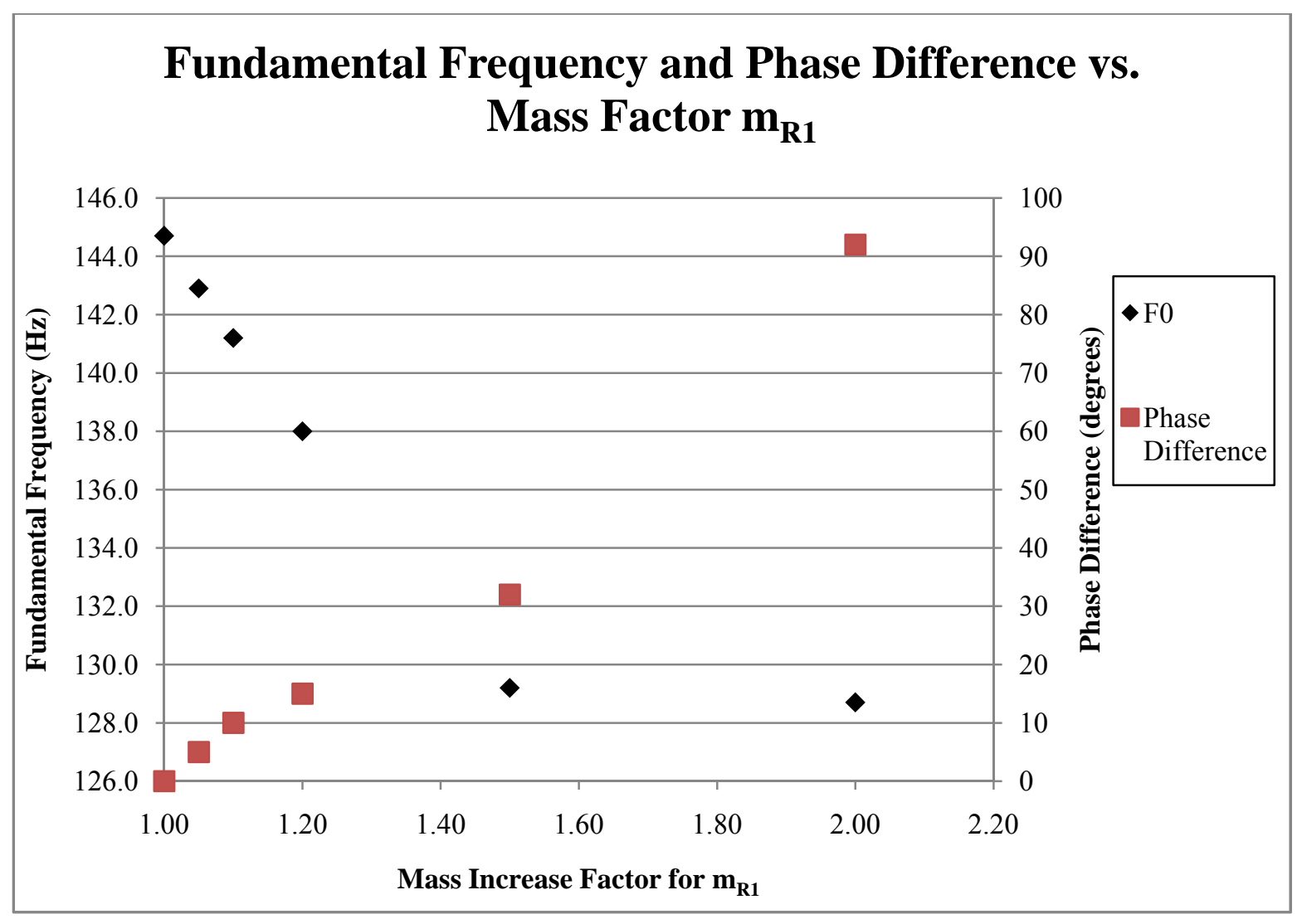

Figure 5-6 Fundamental frequency and phase difference between the right and left vocal folds versus asymmetry factor for $m_{R 1}$

\subsubsection{Asymmetry in the Upper, Right mass}

An increase in the mass of the upper, right oscillator did not result in the same level of asymmetry as observed for the lower mass. This is likely due to the fact that the upper mass is relatively small and most of its motion depends on the energy transferred to it from the lower mass. However, a unique feature was observed in the displacement diagrams. Specifically, the phase difference between the lower, right mass and the upper, right mass (often related to the mucosal wave speed) increased with increasing mass. This is expected due to the greater inertia of the larger upper mass causing it to lag further behind the lower mass. The graphical results are presented in Figures 5-7 and 5-8. 


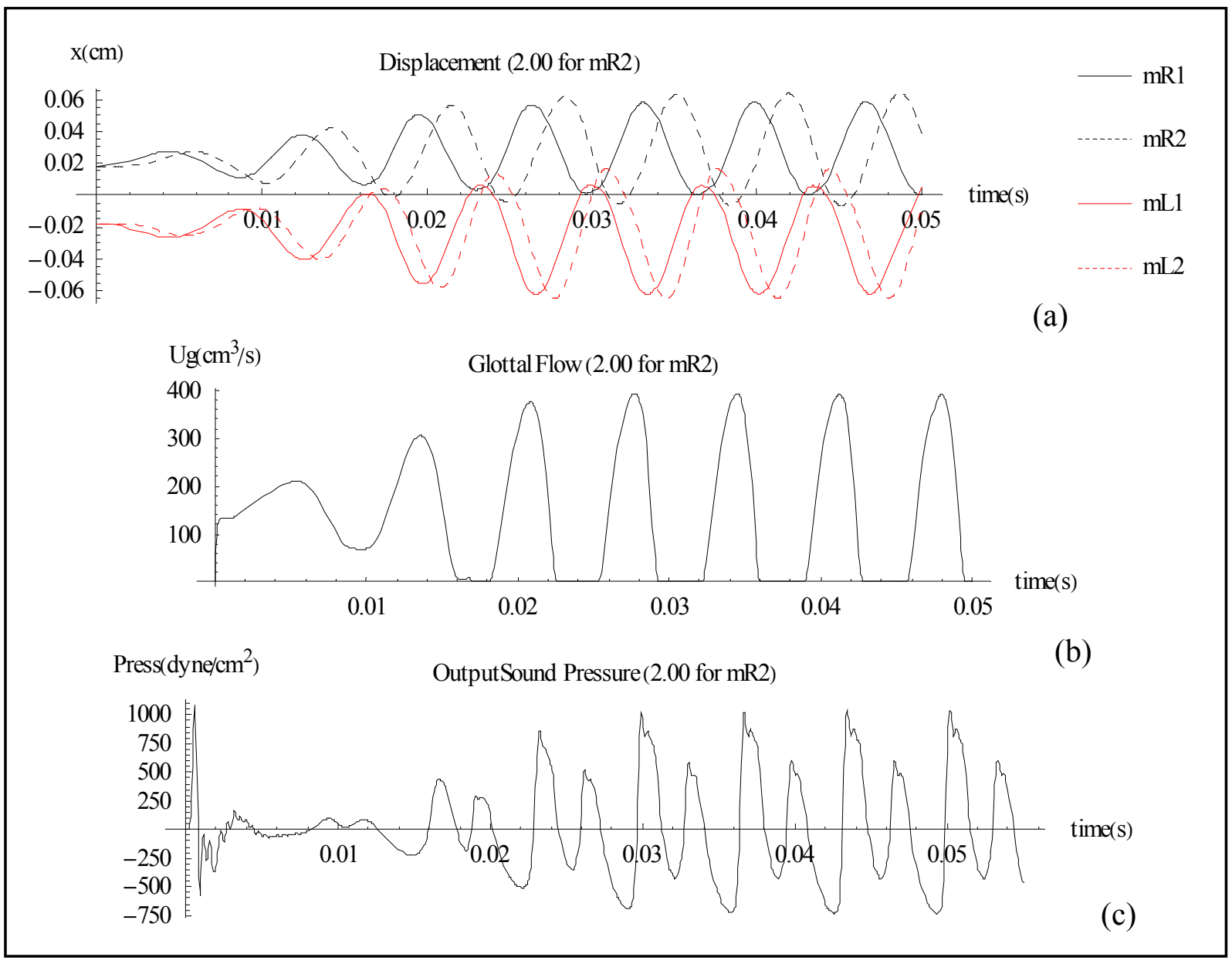

Figure 5-7 Results for an increase in the upper, right mass by a factor of 2.00: (a) oscillator displacements, (b) glottal flow, and (c) output sound pressure level. 

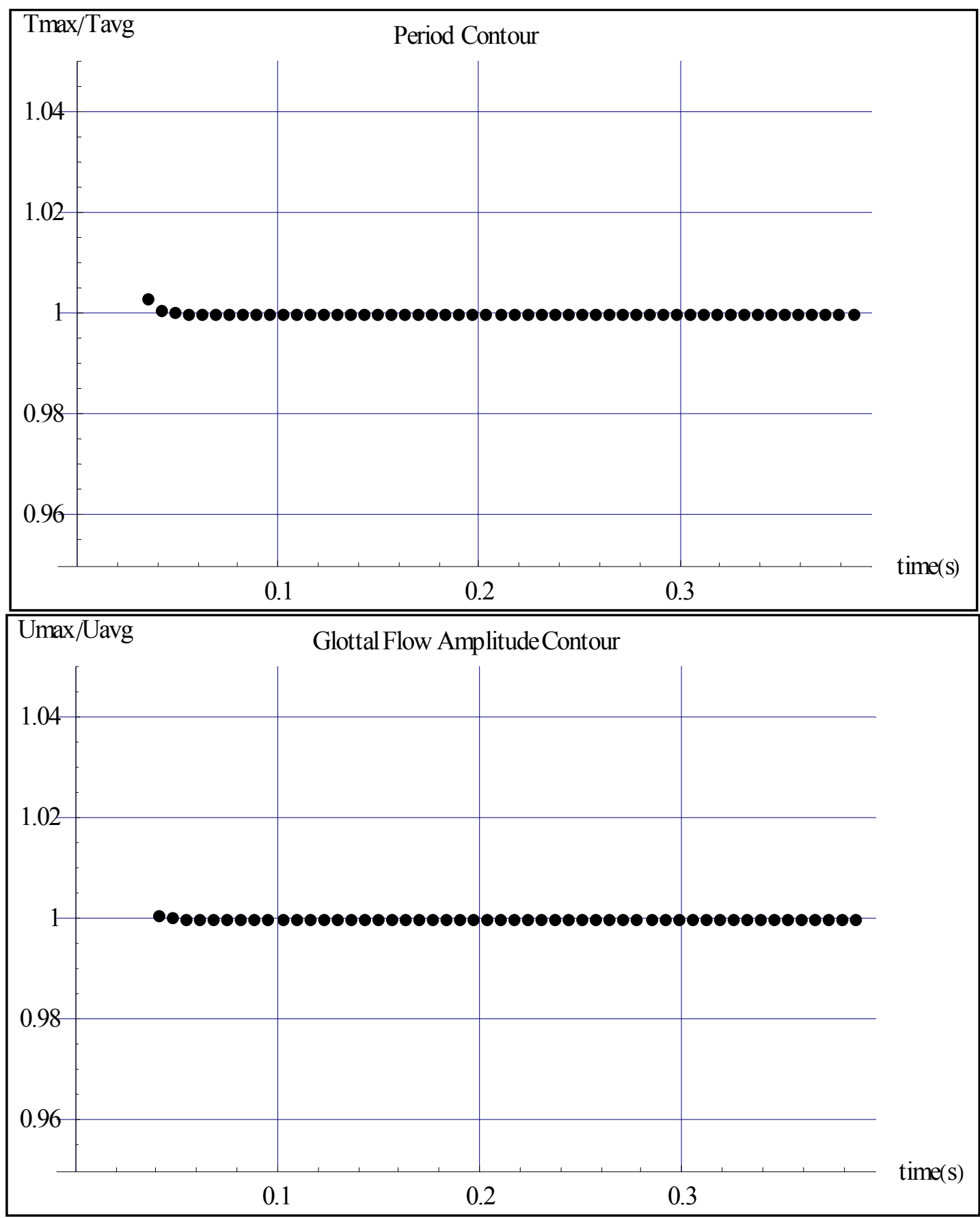

Figure 5-8 Fundamental period contour normalized to the average period and glottal airflow amplitude contour normalized to the average amplitude when $\mathrm{m}_{\mathrm{R} 2}$ is increased by a factor of 2.00 . 
As can be seen from Figure 5-8, no jitter or shimmer was observed even in the most extreme case. The bilateral asymmetry of the oscillator displacements did not result in jitter or shimmer.

\subsubsection{Asymmetry in the Lower, Right Spring Force}

Increasing the lower, right spring constant by varying amounts produced similar results as the case of increasing the lower, right mass. Once again, the vocal folds entrained and found a common equilibrium frequency, which defined the fundamental frequency, and neither jitter nor shimmer was observed. As the $\mathrm{k}_{\mathrm{R} 1}$ factor was increased from 1.05 to 2.00 , the fundamental frequency increased from $146.2 \mathrm{~Hz}$ to $152.1 \mathrm{~Hz}$. The fundamental frequency appears to vary more rapidly with variation of the oscillator masses than with the stiffness factors.

Little additional information is obtained by viewing the glottal flow and output sound pressure level in this case. Alternatively, the graphical results for the oscillator displacements for the 1.05 through 2.00 cases are provided in Figure 5-9. As Figure 5-9 indicates, the amplitude of the right oscillator decreases and the frequency increases with increasing stiffness. Phonation onset time, however, appears to be relatively unaffected. As with the case of increased mass, a phase difference occurs between the left and right vocal folds as the spring stiffness is increased. The vocal folds no longer collide when $\mathrm{k}_{\mathrm{R} 1}$ is increased by a factor of 2.00 . Figure $5-10$ presents the null results for the jitter and shimmer calculations for the factor of 2.00 increase.

\subsubsection{Asymmetry in the Upper, Right Spring Force}

Once again, neither jitter nor shimmer was observed for any level of increase in the upper, right spring constant, $\mathrm{k}_{\mathrm{R} 2}$. This is evident in Figure 5-11, which represents the most extreme case of a factor of 2.00 increase. 

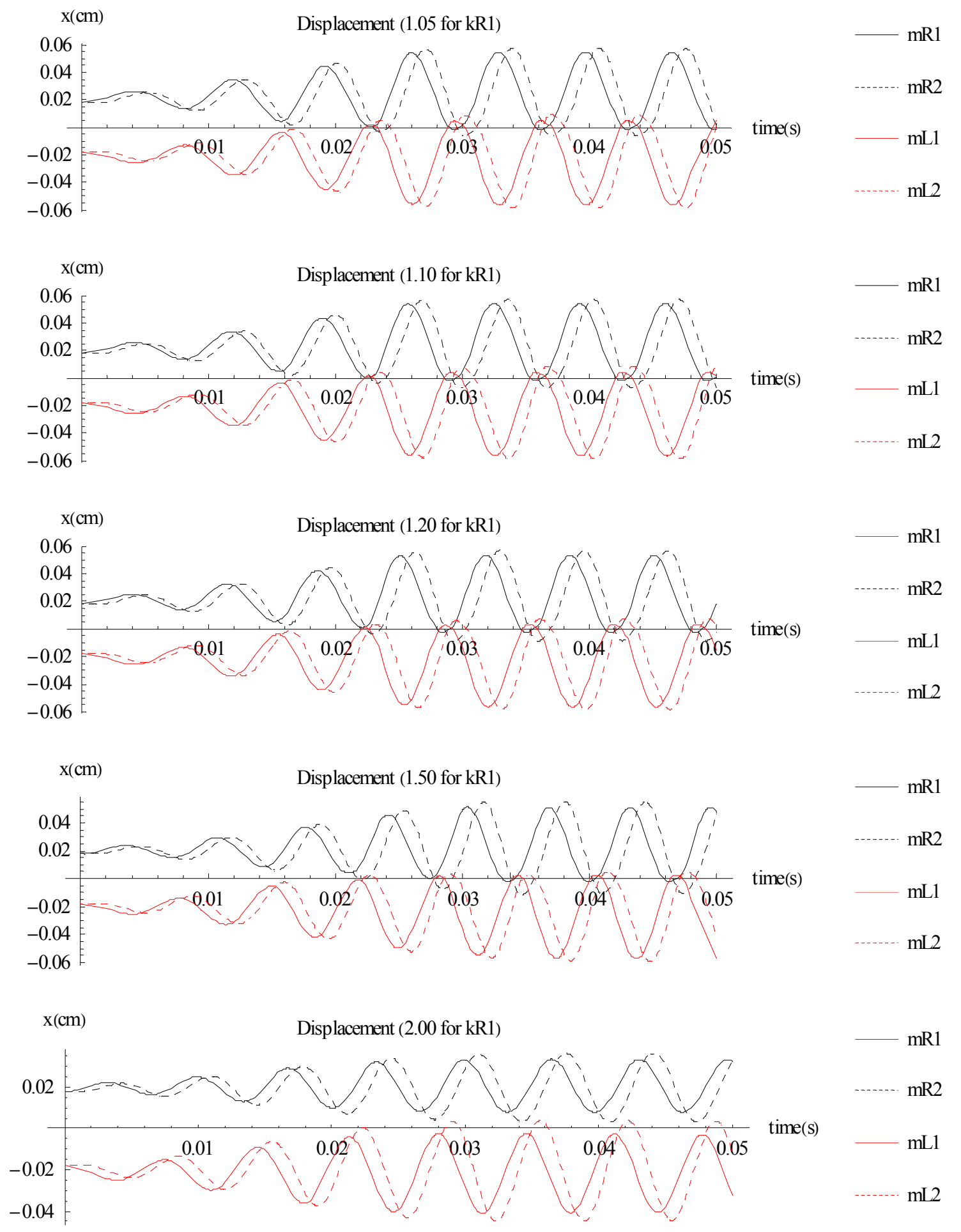

Figure 5-9 Displacement diagrams for increasing $k_{R 1}$ by factors of $1.05,1.10,1.20,1.50$, and 2.00 (from top to bottom). 

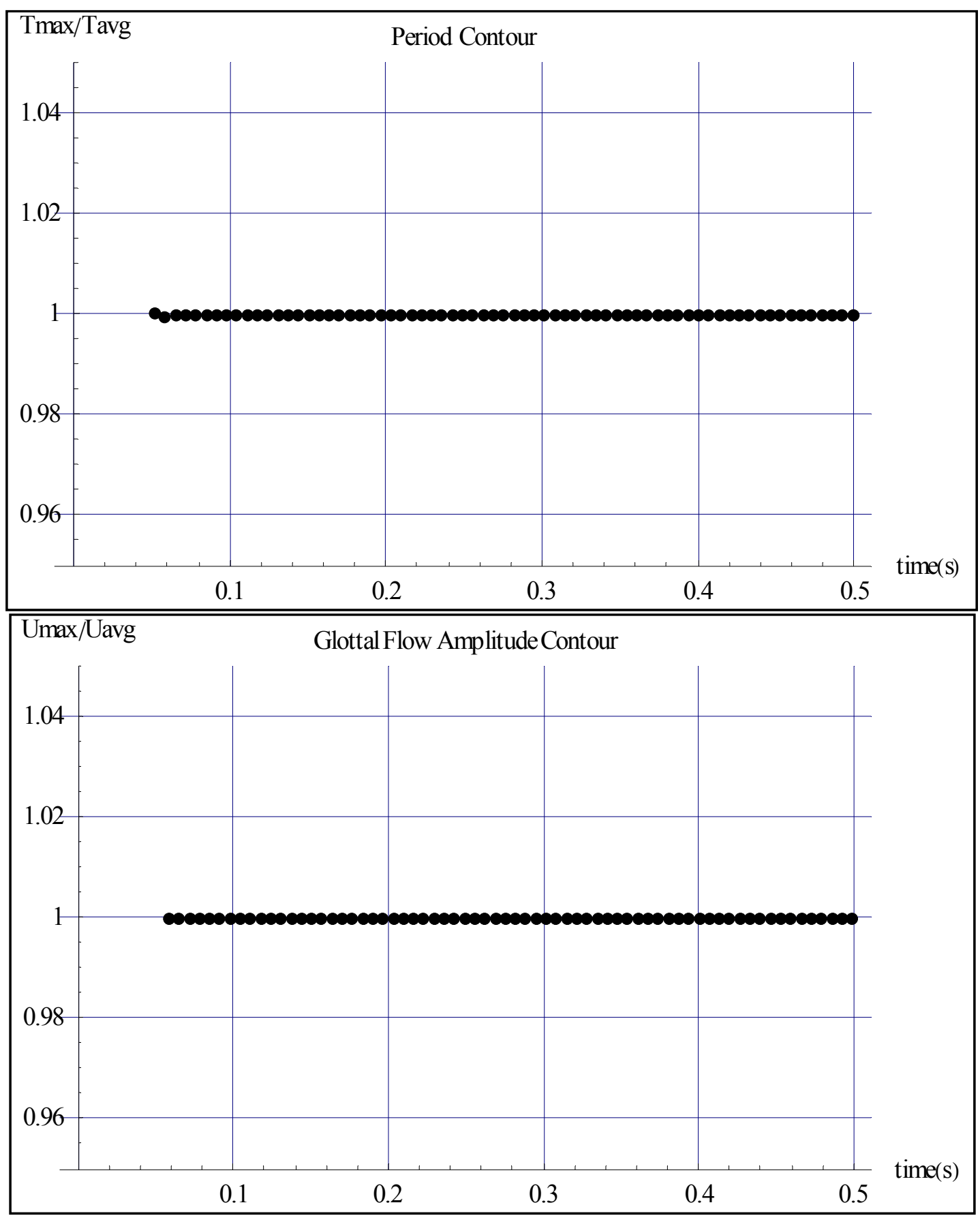

Figure 5-10 Fundamental period contour normalized to the average period and glottal airflow amplitude contour normalized to the average amplitude when $\mathrm{k}_{\mathrm{R} 1}$ is increased by a factor of 2.00 . 

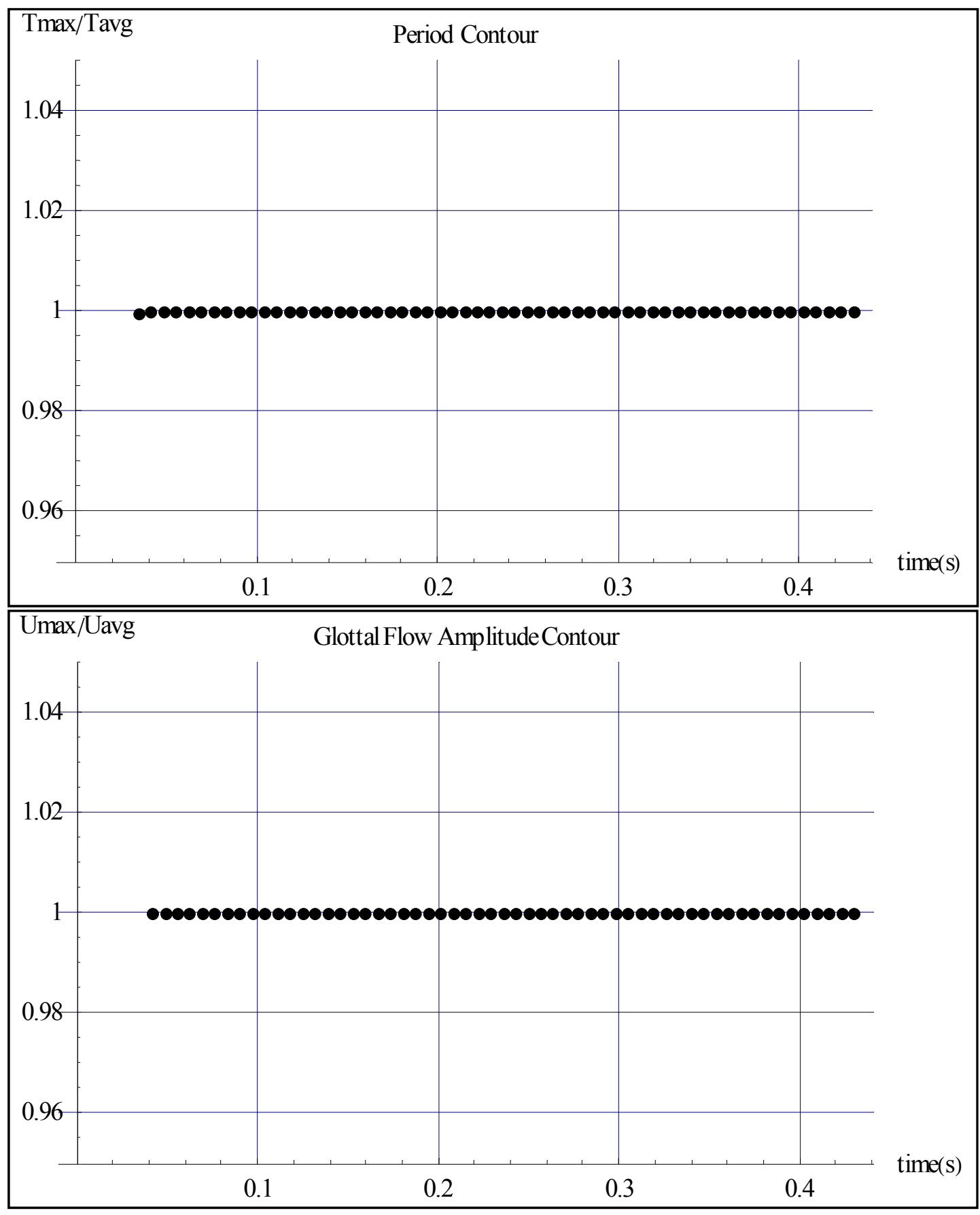

Figure 5-11 Fundamental period contour normalized to the average period and glottal airflow amplitude contour normalized to the average amplitude when $\mathrm{k}_{\mathrm{R} 2}$ is increased by a factor of 2.00 . 
Considering that $\mathrm{k}_{\mathrm{R} 2}$ is normally ten times smaller than $\mathrm{k}_{\mathrm{R} 1}$, it is not surprising that increases in $k_{R 2}$ have relatively subtle effects on the oscillator displacements. As $k_{R 2}$ is increased, the amplitude of the upper, right oscillator is decreased due to the increase in stiffness. Although a decrease in amplitude is expected, an effect contrary to expectations is observed in the frequency in that it is reduced from $144.6 \mathrm{~Hz}$ to $144.1 \mathrm{~Hz}$ as $\mathrm{k}_{\mathrm{R} 2}$ is increased from factors of 1.00 to 2.00 . One might expect that an increase in any spring constant would increase the frequency, because of the standard relation, $\omega=\sqrt{k / m}$, but some feature of the non-linear coupled, oscillators overcompensates for the increase in $\mathrm{k}_{\mathrm{R} 2}$. The graphical results for the oscillator displacements when $\mathrm{k}_{\mathrm{R} 2}$ is increased by factors of 1.05 to 2.00 are provided in Figure $5-12$.

\subsubsection{Asymmetry in the Right, Coupling Spring Force}

The time-independent modification of the right, coupling spring constant had no effect on the cycle-to-cycle period nor did it produce any cycle-to-cycle change of amplitude. This is evident from the null results presented in the fundamental period contour and the glottal airflow contour plots as $\mathrm{k}_{\mathrm{Rc}}$ is increased by a factor of 2.00, presented in Figure 5-13.

Similar to the previous cases, it is most instructive to review the features of the oscillator displacement graphs. As the magnitude of the right, coupling spring is increased by a factor of 1.05 to 2.00 , the amplitude of the right vocal fold decreases, the mucosal wave speed increases (vertical phase difference), and the system frequency decreases (perhaps surprisingly) from 144.6 Hz to $142.2 \mathrm{~Hz}$. Also, the onset time increases with increasing $\mathrm{k}_{\mathrm{Rc}}$; an effect consistent with one's expectations about the role of the excitation of surface waves as necessary for the self-oscillation of the vocal folds. As the medial surface becomes less flexible, it becomes more 


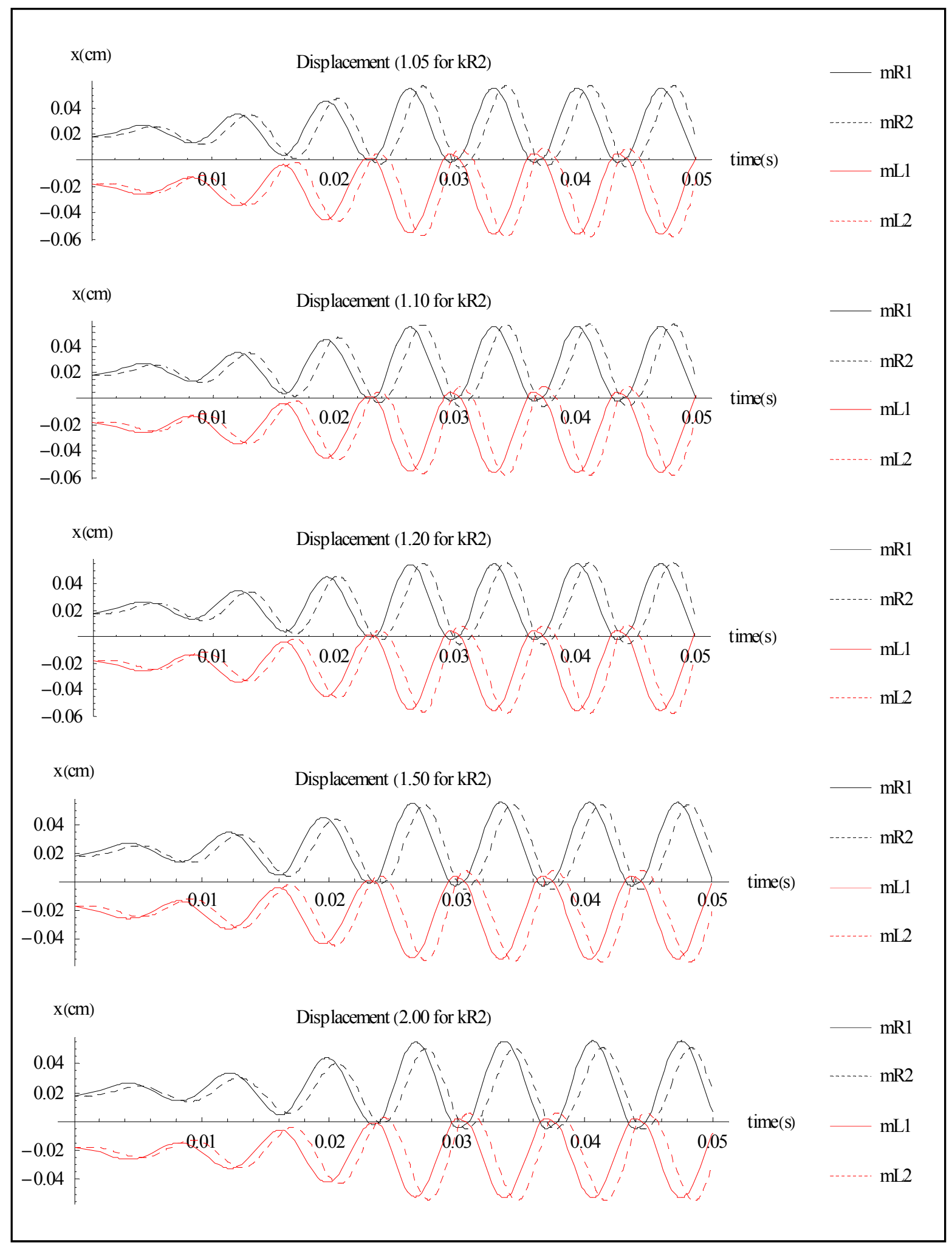

Figure 5-12 Displacement diagrams for increasing $\mathrm{k}_{\mathrm{R} 2}$ by factors of $1.05,1.10,1.20,1.50$, and 2.00 (from top to bottom). 

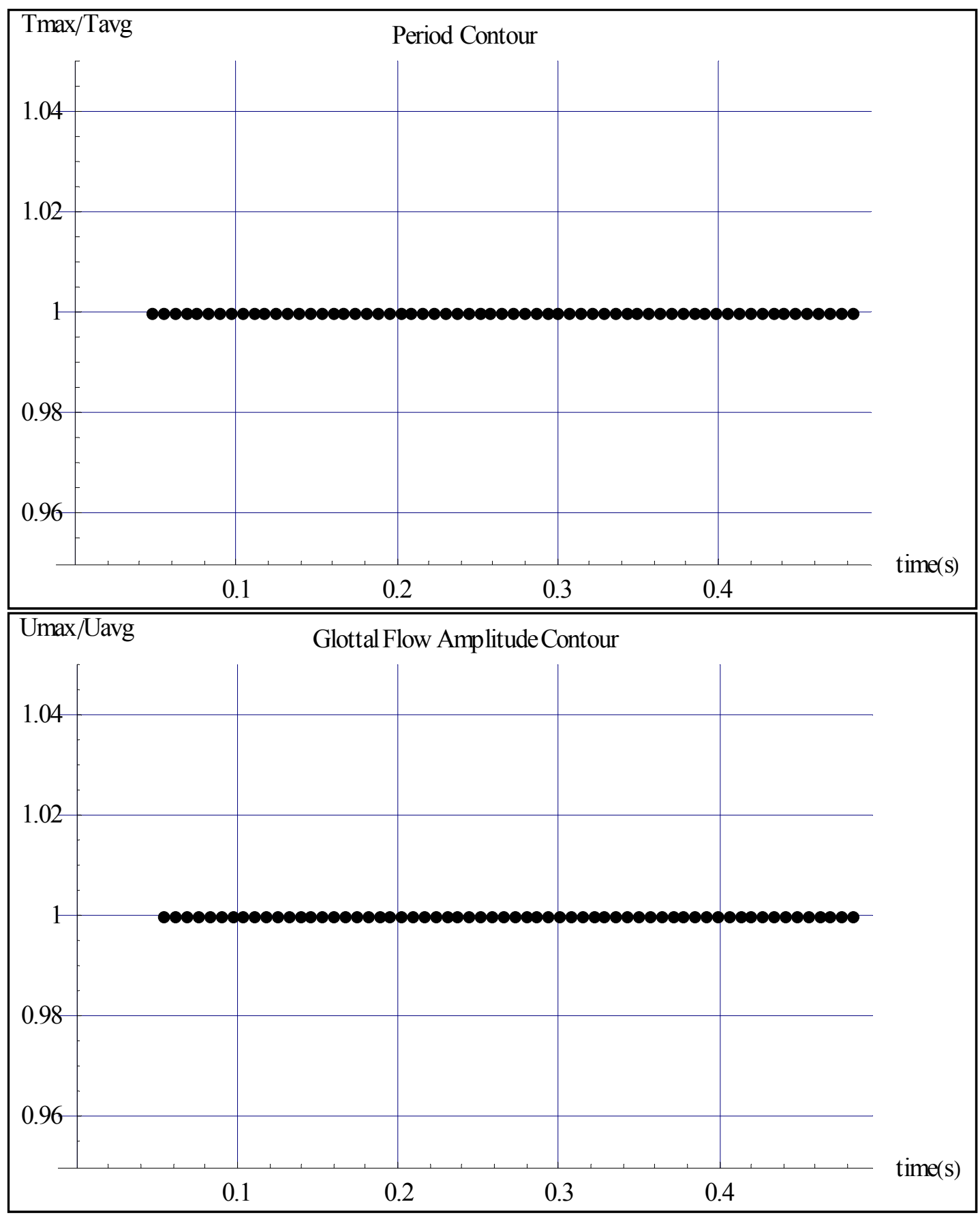

Figure 5-13 Fundamental period contour normalized to the average period and glottal airflow amplitude contour normalized to the average amplitude for the case of $k_{R c}$ increased by a factor of 2.00 


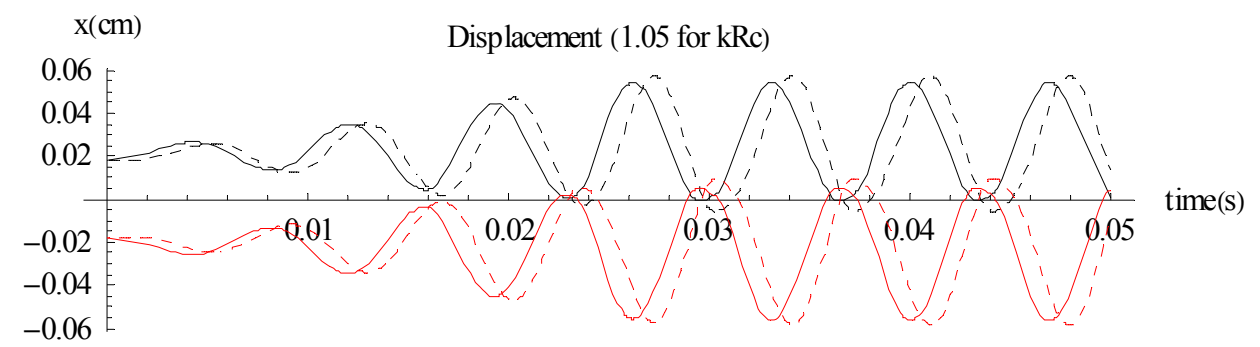

$-\mathrm{mR} 1$

$\mathrm{x}(\mathrm{cm}) \quad$ Displacement $(1.10$ for $\mathrm{kRc})$
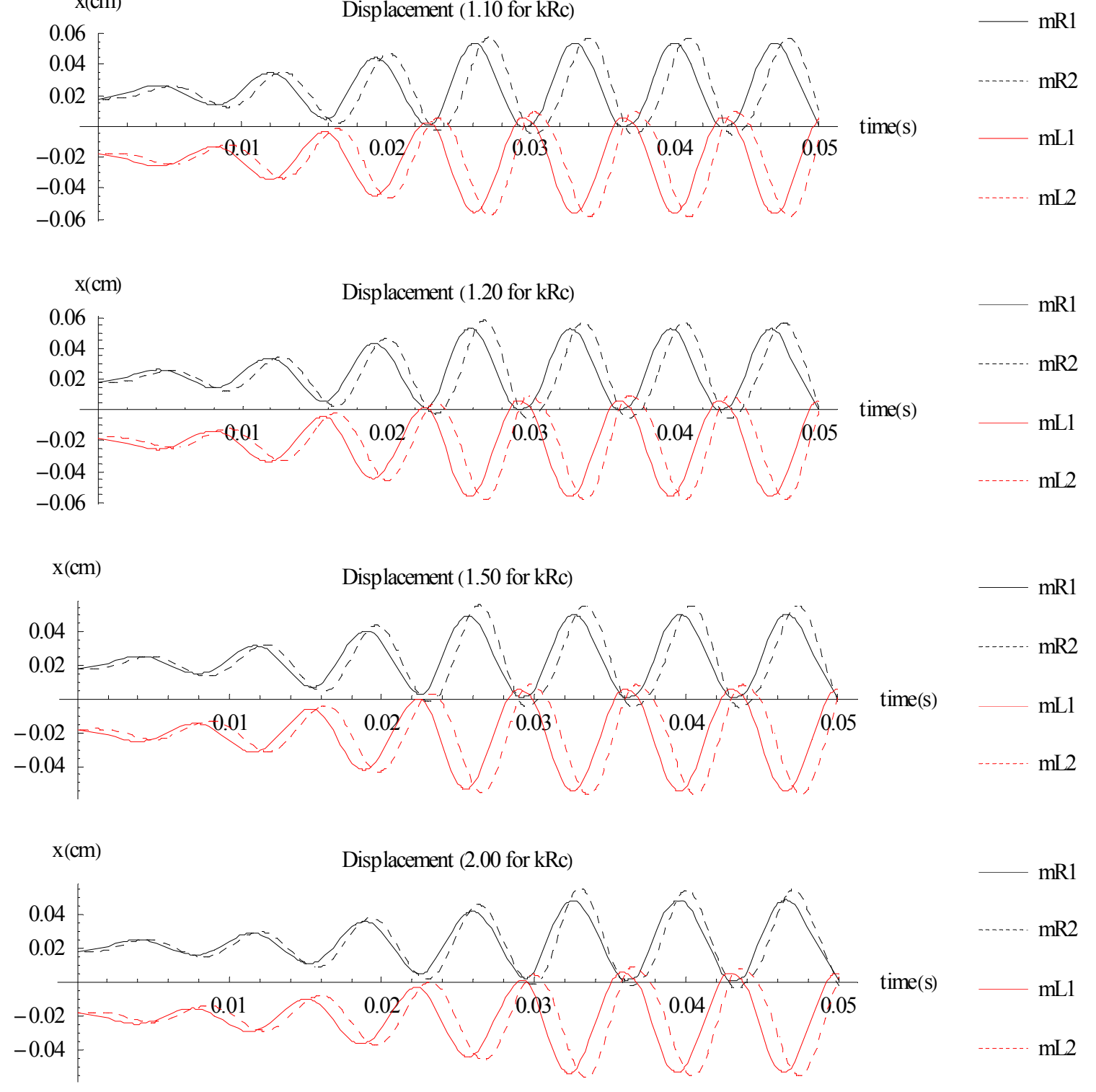

Figure 5-14 Displacement diagrams for increasing $\mathrm{k}_{\mathrm{Rc}}$ by factors of $1.05,1.10,1.20,1.50$, and 2.00 (from top to bottom) 
difficult to excite a surface wave, which is necessary to give the alternating converging and diverging shapes. As stated earlier, this sequence of alternating shapes is necessary to give the pushes and pulls that transfer the requisite energy for self-oscillation (Fulcher, Scherer, Melnykov et al. 2006).

\subsection{Hypothesis \#2: Time-dependent Physical Asymmetry}

As mentioned in the methodology, the modeling of time-dependent physical asymmetries required that some of the constant parameters be converted to time-dependent functions. The nature and basis of the time variations applied here are also discussed in the methodology. The effects of these new time dependencies are presented in the following subsections. In cases where jitter and shimmer were detected, it should be kept in mind that in natural sounding voice the jitter factor (JF) is approximately $0.5 \%$ and the amplitude variability index (AVI) for shimmer is approximately -0.1 (Baken and Orlikoff 2000).

\subsubsection{Random Asymmetry in the Lower, Right Spring Constant}

The lower, right spring constant $\left(\mathrm{k}_{\mathrm{R} 1}\right)$ was converted to a randomly fluctuating function of time in general accordance with the work by Titze (1991). Based on this procedure, both the period and amplitude of $\mathrm{k}_{\mathrm{R} 1}$ were varied randomly. The period of this perturbation was maintained at an average of $40 \mathrm{~ms}$ over 100 motor units for every model run, while the amplitude of the perturbation was allowed to vary from $0.5 \%$ to $50 \%$ of the typical constant value for $k_{\mathrm{R} 1}$ of 80,000 dyne $/ \mathrm{cm}$. Examples of the time-dependence of $\mathrm{k}_{\mathrm{R} 1}$ for $1 \%$ and $25 \%$ variation are presented in Figure 5-15.

The random, time-dependent variations in $\mathrm{k}_{\mathrm{R} 1}$ resulted in measurable amounts of jitter and shimmer. For the sake of brevity, only one case is presented here with the oscillator displacements, glottal flow, output sound pressure, fundamental period contour, and glottal 
airflow contour given for the 25\% case in Figures 5-16 and 5-17. As can be observed in Figure 5-16(b), there appear to be slight variations in the amplitudes of the glottal flow pulse and the acoustic pressure signal. The fundamental period contour and the glottal airflow contour, both normalized to the respective averages, are provided in Figure 5-17. These plots show that the variations are obviously present and appear to be completely random.

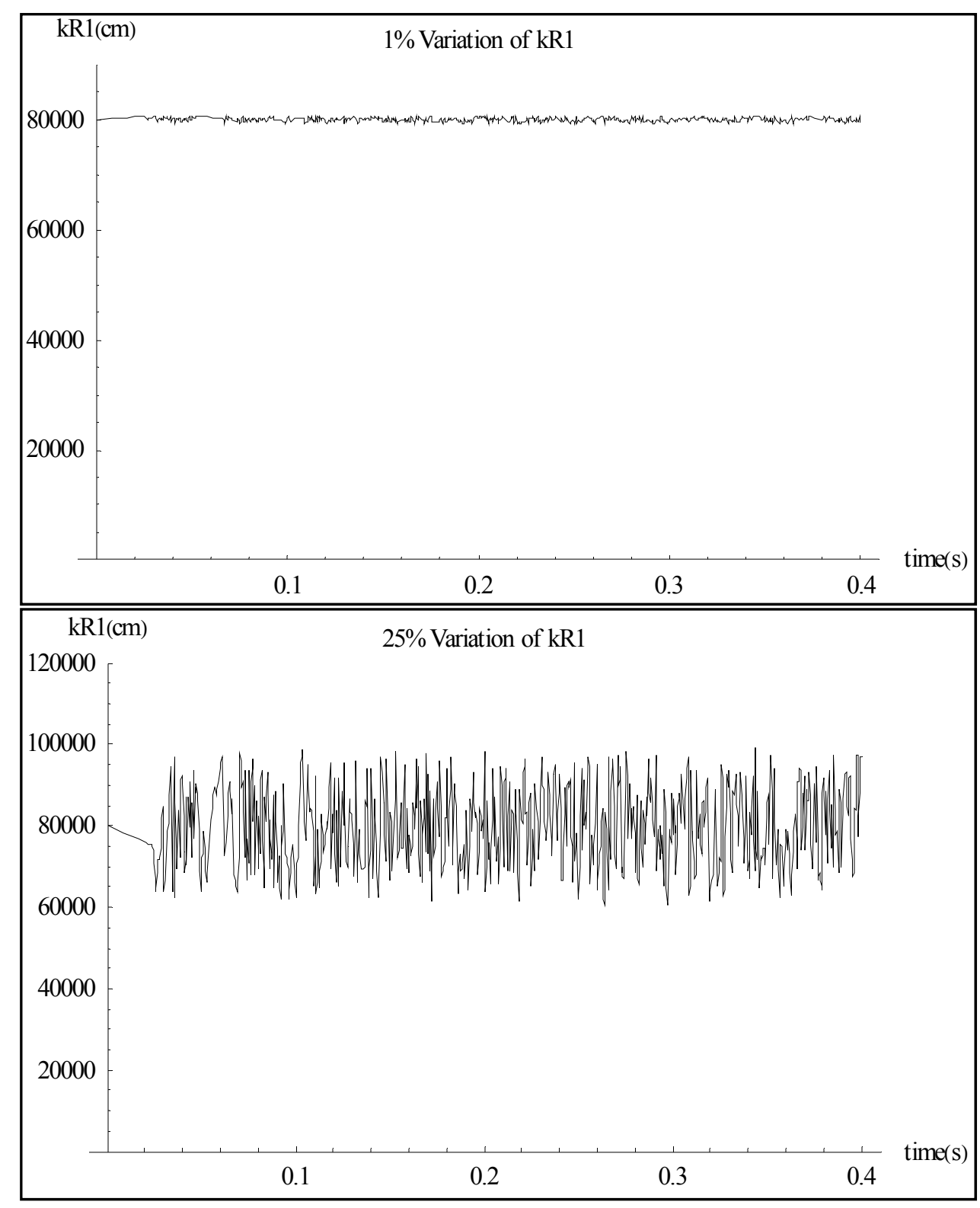

Figure 5-15 Time-dependence of $k_{R 1}$ for $1 \%$ (top) and $25 \%$ (bottom) random variations 


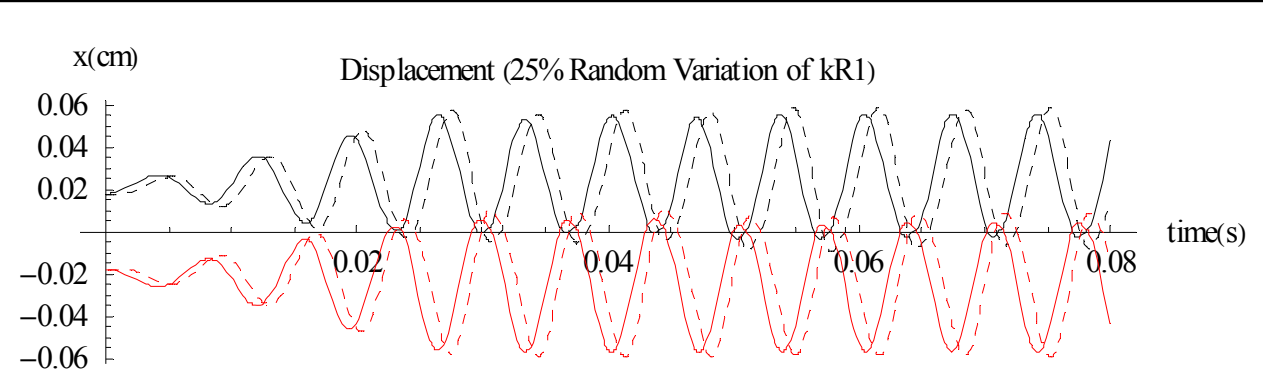

$\mathrm{mR} 1$

$\mathrm{mR} 2$ $\mathrm{mL} 1$ $\mathrm{mL} 2$

$\mathrm{Ug}\left(\mathrm{cm}^{3} / \mathrm{s}\right)$

Glottal Flow (25\% Random Variation of kR1)

(a)

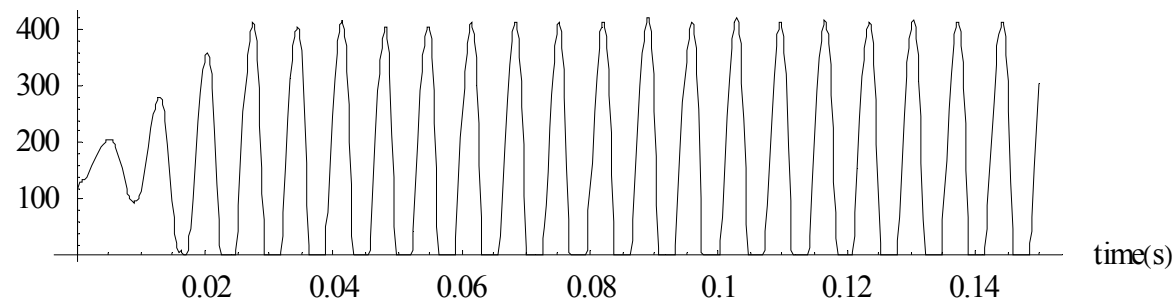

Press (dyne/ $\left./ \mathrm{cm}^{2}\right)$

OutputSound Pressure(25\% Random Variation of $k R 1)$

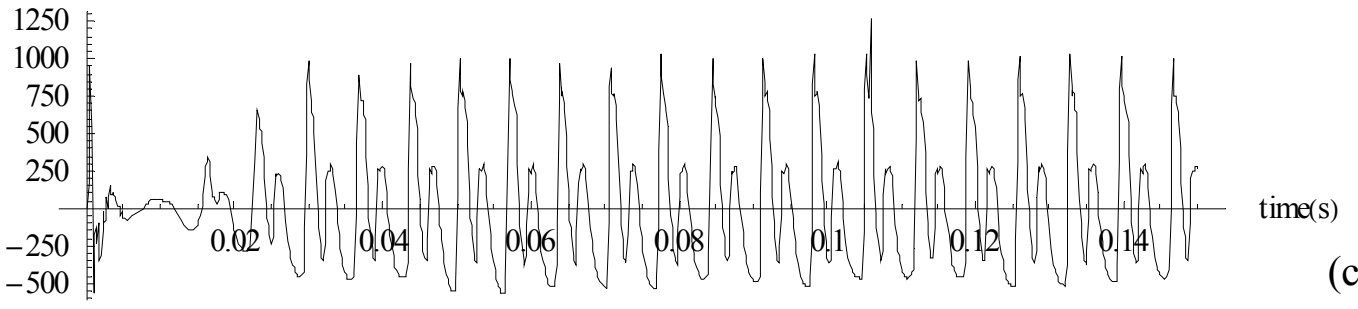

Figure 5-16 Results for random fluctuations in the lower, right spring force $\mathrm{k}_{\mathrm{R} 1}$ by $25 \%$, (a) oscillator displacements, (b) glottal flow, and (c) output sound pressure level. 

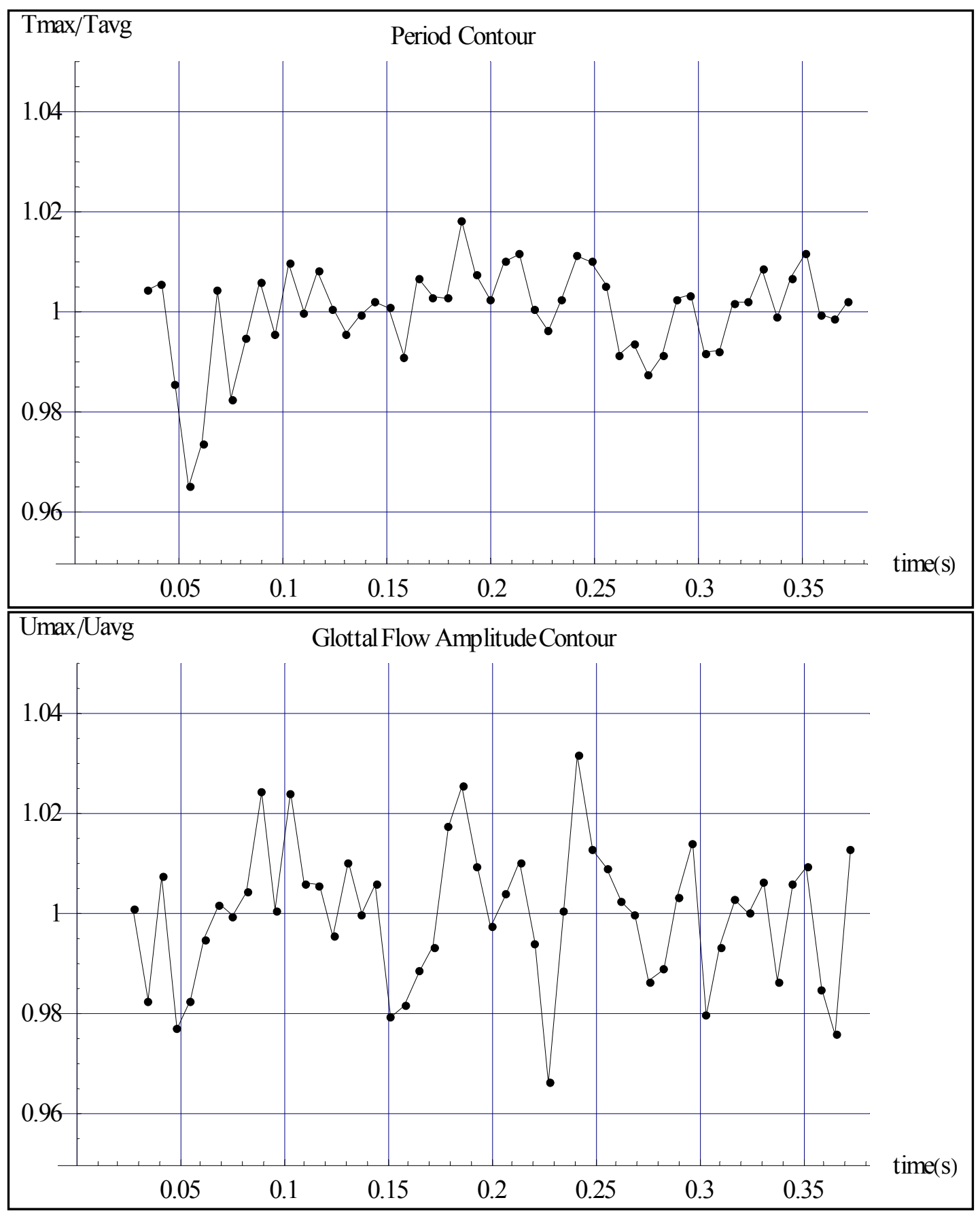

Figure 5-17 Fundamental period contour normalized to the average period and glottal airflow amplitude contour normalized to the average amplitude for random fluctuations of the lower, right spring constant $\mathrm{k}_{\mathrm{R} 1}$ by a factor of $25 \%$

The JF and the AVI were calculated for each case. The JF ranged from $0.02 \%$ to $1.69 \%$, while the AVI ranged from -4.15 to -0.19 . There is a linear correlation between the jitter and the shimmer of 0.29. It is interesting to note that the jitter factor appears to behave linearly with 
respect to the $k_{R 1}$ factor and the AVI behaves logarithmically, which is expected since the AVI is a logarithmic function. The natural level of jitter $(0.5 \%)$ would require a fluctuation in $\mathrm{k}_{\mathrm{R} 1}$ of approximately $15 \%$. Physiologically, this variation would be in the form of unsteady muscle contractions or, possibly, a lack of motor control. Typically, the variation in the force of tetanic muscle contraction is on the order of $1 \%$. Consequently, it appears that tetanic contraction fluctuation in $\mathrm{k}_{\mathrm{R} 1}$ alone is insufficient to account for natural jitter. The results are presented graphically in Figure 5-18.

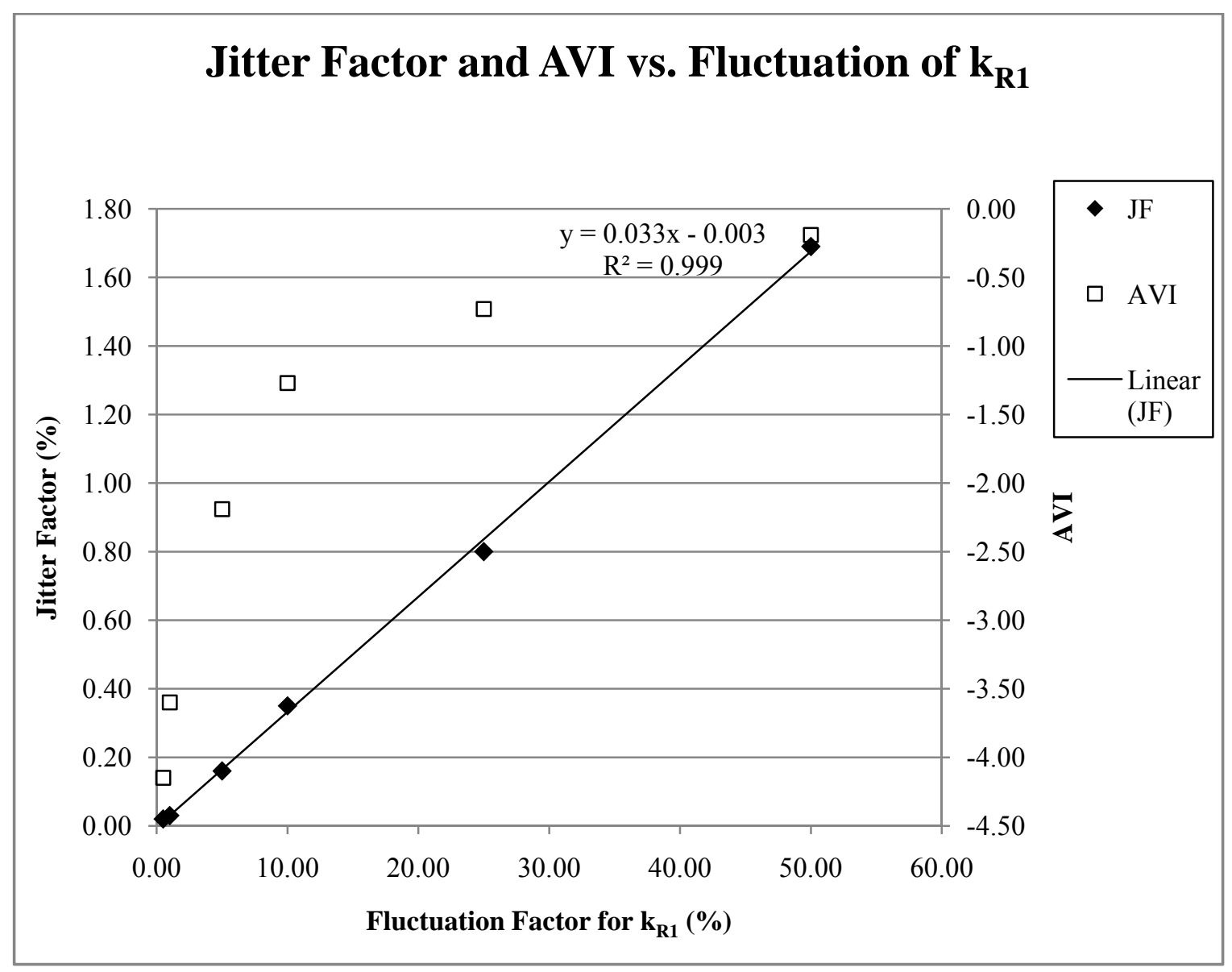

Figure 5-18 Jitter Factor and Amplitude Variability Index versus the magnitude of random fluctuations of $k_{R 1}$. 


\subsubsection{Random Asymmetry in the Lower, Right and Left Spring Constants}

The spring constants of the lower masses on both the left and right hand sides $\left(\mathrm{k}_{\mathrm{R} 1}\right.$ and $\mathrm{k}_{\mathrm{L} 1}$ ) were allowed to vary randomly, as described above, to simulate fluctuations of the effective tension of the tissue in motion due to the vocalis muscle on both sides of the larynx. The graphical results are provided for the $25 \%$ case for comparison to the results of the variation of $\mathrm{k}_{\mathrm{R} 1}$ alone given in the previous section. The graphs for the oscillator displacements, glottal airflow, and output sound pressure are provided in Figure 5-19. The fundamental period contour and glottal airflow amplitude contour are provided in Figure 5-20.

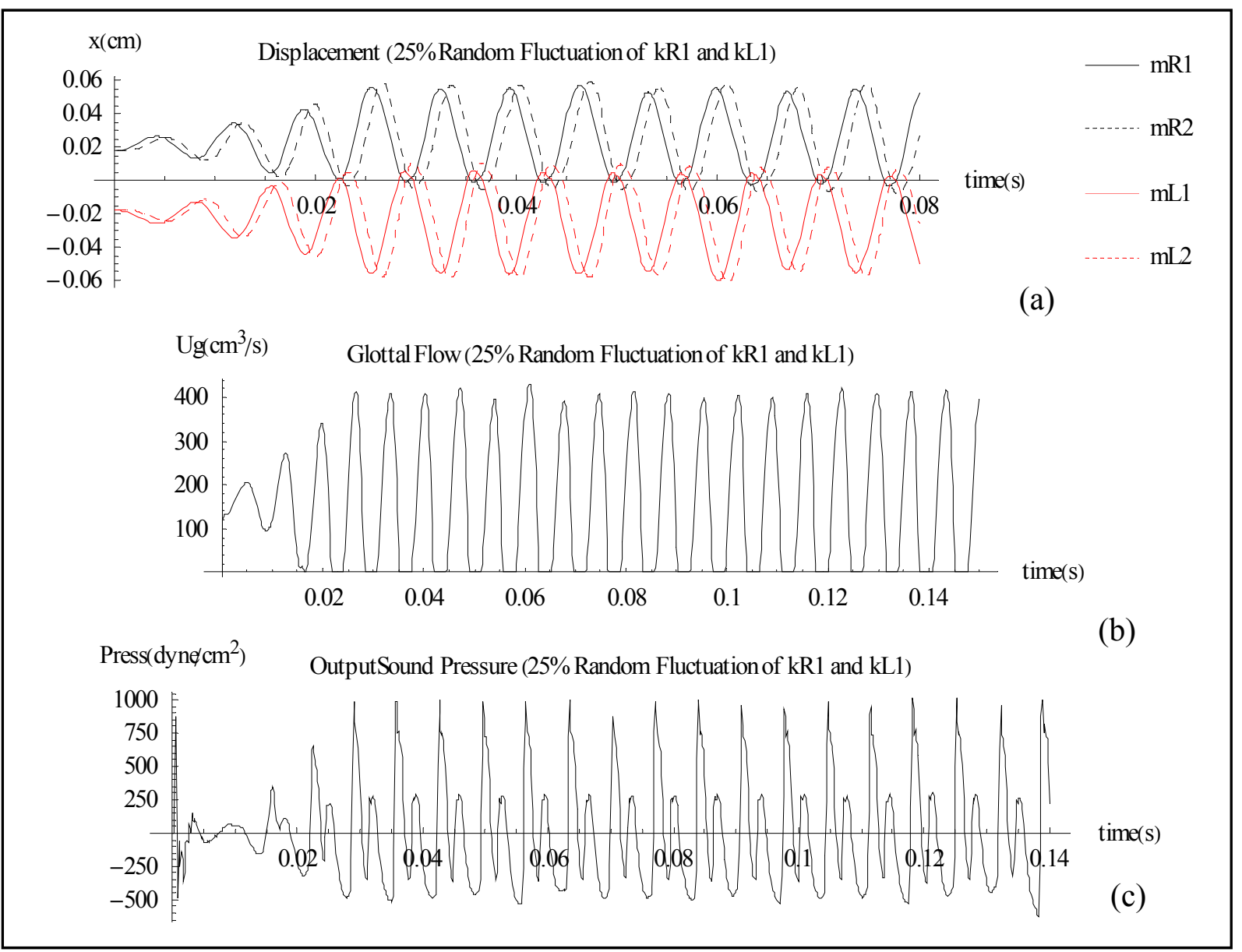

Figure 5-19 Results for random fluctuations in the lower, right and left spring forces, $\mathrm{k}_{\mathrm{R} 1}$ and $\mathrm{k}_{\mathrm{L} 1}$, by $25 \%$, (a) oscillator displacements, (b) glottal flow, and (c) output sound pressure level. 


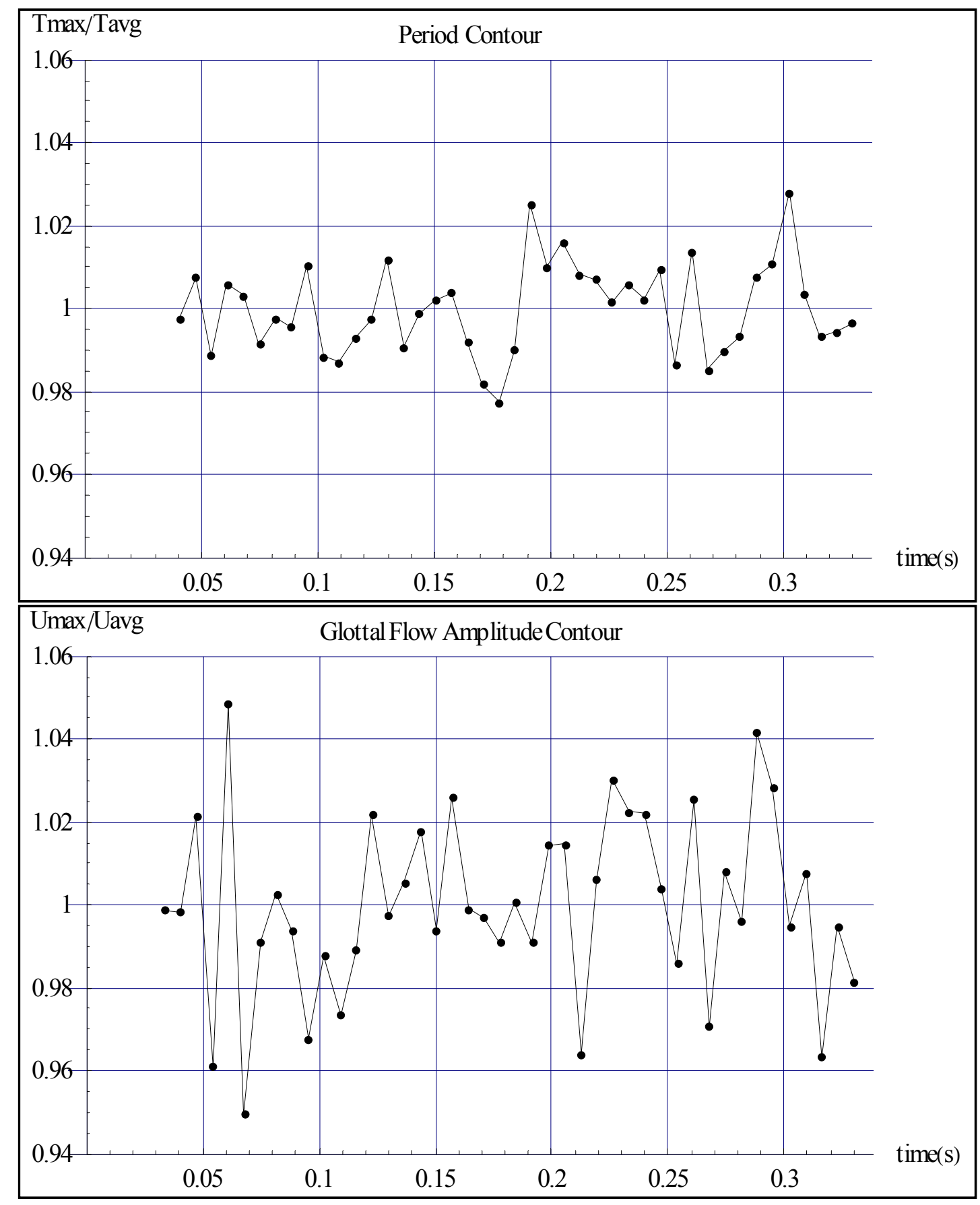

Figure 5-20 Fundamental period contour normalized to the average period and glottal airflow amplitude contour normalized to the average amplitude for random fluctuations of the lower, right spring constants $\mathrm{k}_{\mathrm{R} 1}$ and $\mathrm{k}_{\mathrm{L} 1}$ by a factor of $25 \%$

The variations in the amplitudes of the glottal flow are more obvious in this case than the case of $k_{R 1}$ fluctuation only. Also, the increased levels of jitter and shimmer are apparent when 
comparing Figure 5-20 to Figure 5-17. The numerical values provided by the JF and AVI are also informative and are provided in Figure 5-21 with respect to the fluctuation levels of $\mathrm{k}_{\mathrm{R} 1}$ and $\mathrm{k}_{\mathrm{L} 1}$. The JF values range from $0.03 \%$ to $2.59 \%$ and the AVI values range from -3.77 to 0.27 . Similar to the previous case, there is a positive linear correlation between jitter and shimmer of 0.25. Again, there is a linear relationship between JF and the fluctuation factor. The normal voice jitter of $0.5 \%$ is achieved in this scenario with a fluctuation in the muscle tension of $+/-$ $10 \%$. This unsteadiness in muscle tension is still much greater than would be expected under normal circumstances. Unreasonable levels of close to $35 \%$ fluctuation of the muscle tension resulted in the AVI value for normal voice (approximately -0.1). It appears that tetanic contraction fluctuation in both $\mathrm{k}_{\mathrm{R} 1}$ and $\mathrm{k}_{\mathrm{L} 1}$ is insufficient to cause normal jitter and shimmer.

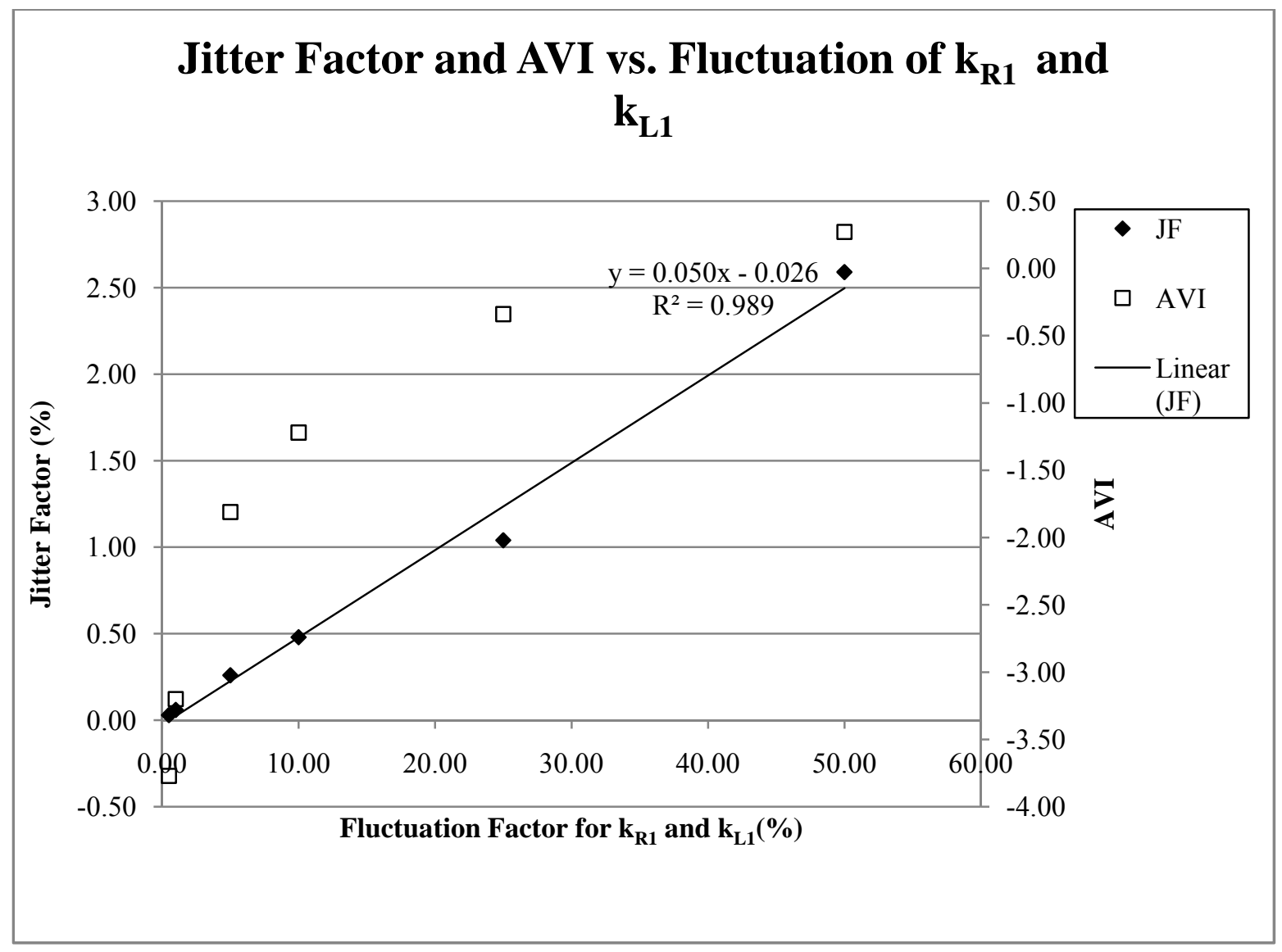

Figure 5-21 Jitter Factor and Amplitude Variability Index versus the magnitude of random fluctuations of $\mathrm{k}_{\mathrm{R} 1}$ and $\mathrm{k}_{\mathrm{L} 1}$. 


\subsubsection{Random Asymmetry in All Spring Constants}

All six spring constants, $\mathrm{k}_{\mathrm{R} 1}, \mathrm{k}_{\mathrm{L} 1}, \mathrm{k}_{\mathrm{R} 2}, \mathrm{k}_{\mathrm{L} 2}, \mathrm{k}_{\mathrm{Rc}}$, and $\mathrm{k}_{\mathrm{Lc}}$ were converted to functions of time with the random fluctuations described previously. Surprisingly, very little difference was detected between this case and the case where only $\mathrm{k}_{\mathrm{R} 1}$ and $\mathrm{k}_{\mathrm{L} 1}$ were allowed to fluctuate. For comparison purposes, graphs for the $25 \%$ random fluctuation case are presented in Figures 5-22 and 5-23.

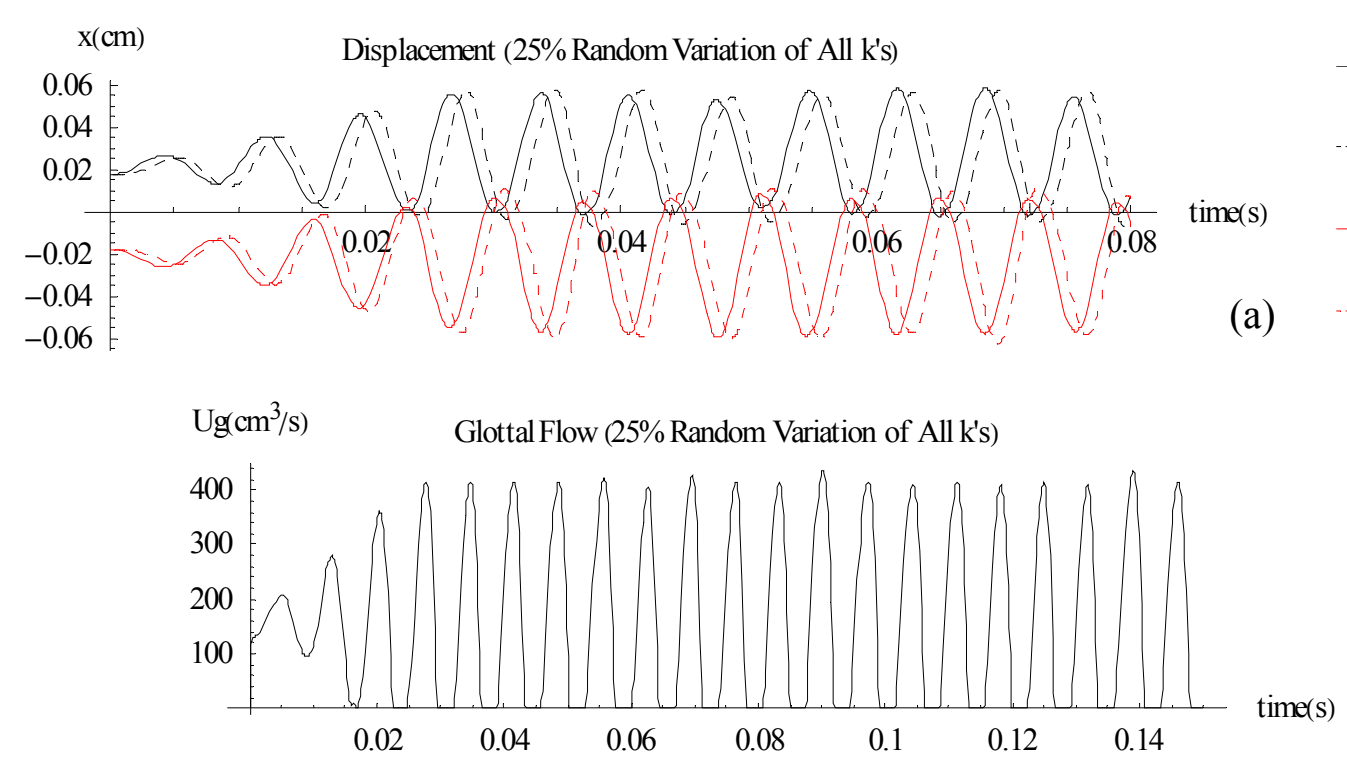

Press (dynę $\left.\mathrm{cm}^{2}\right) \quad$ OutputSound Pressure (25\% Random Variation of All k's)

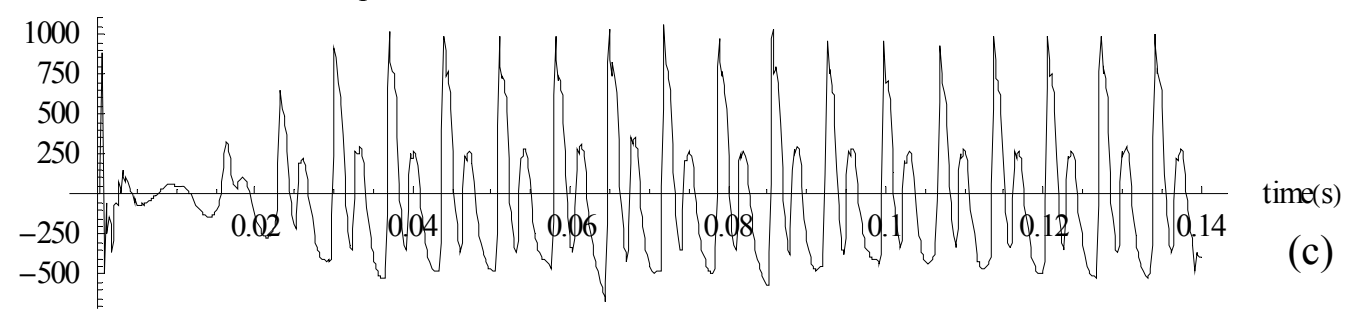

Figure 5-22 Results for random fluctuations in all of the spring forces by $25 \%$, (a) oscillator displacements, (b) glottal flow, and (c) output sound pressure level. 

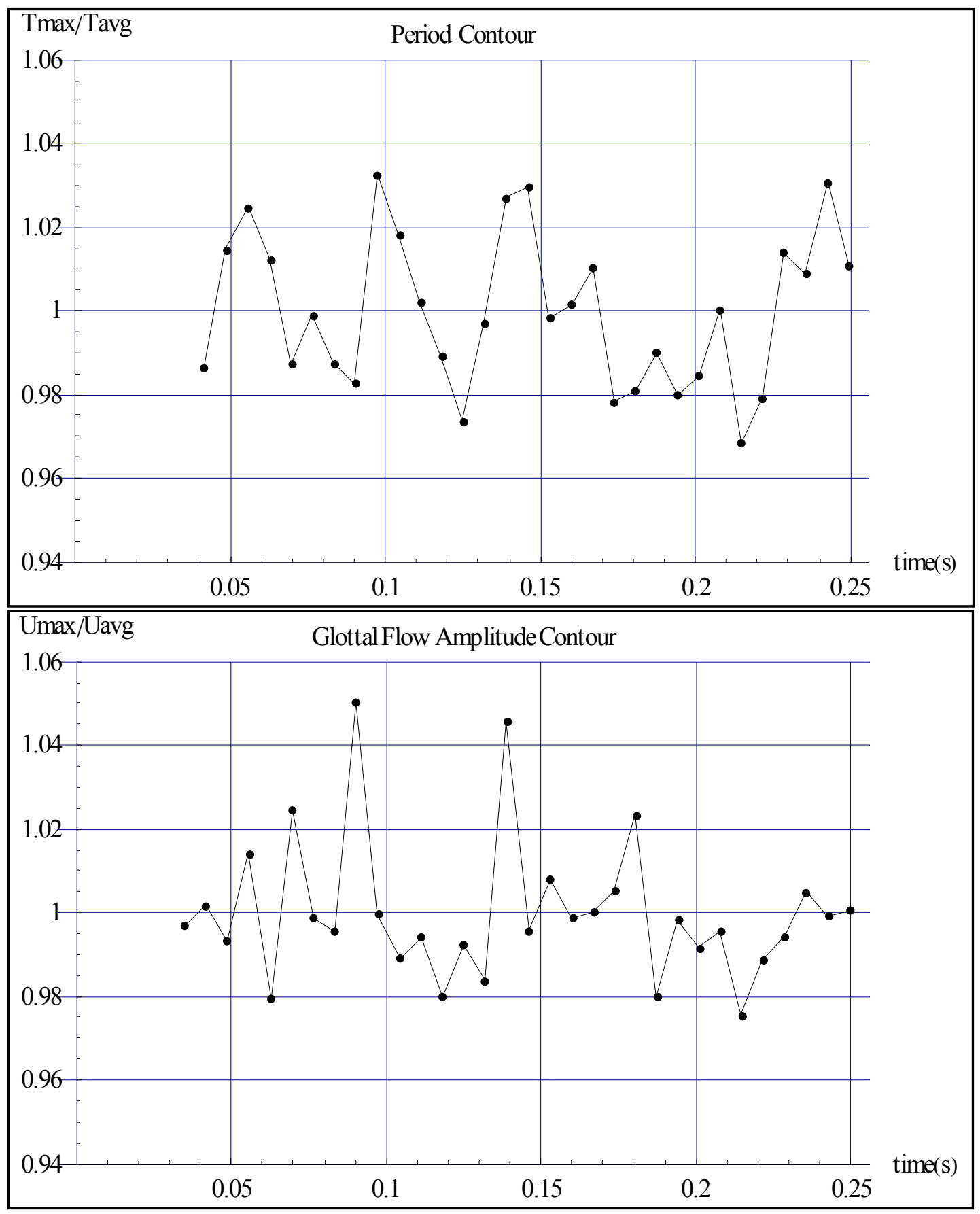

Figure 5-23 Fundamental period contour normalized to the average period and glottal airflow amplitude contour normalized to the average amplitude for random fluctuations of all spring constants by a factor of $25 \%$.

The JF values for this case ranged from $0.03 \%$ to $2.46 \%$ and the AVI values ranged from -3.60 to 0.38 . There is a positive linear correlation of jitter and shimmer of 0.45 . These results are very similar to the case where $\mathrm{k}_{\mathrm{R} 1}$ and $\mathrm{k}_{\mathrm{L} 1}$ were allowed to fluctuate, but it appears that there 
was a measurable increase in shimmer for this case. The JF and AVI results are presented in Figure 5-24.

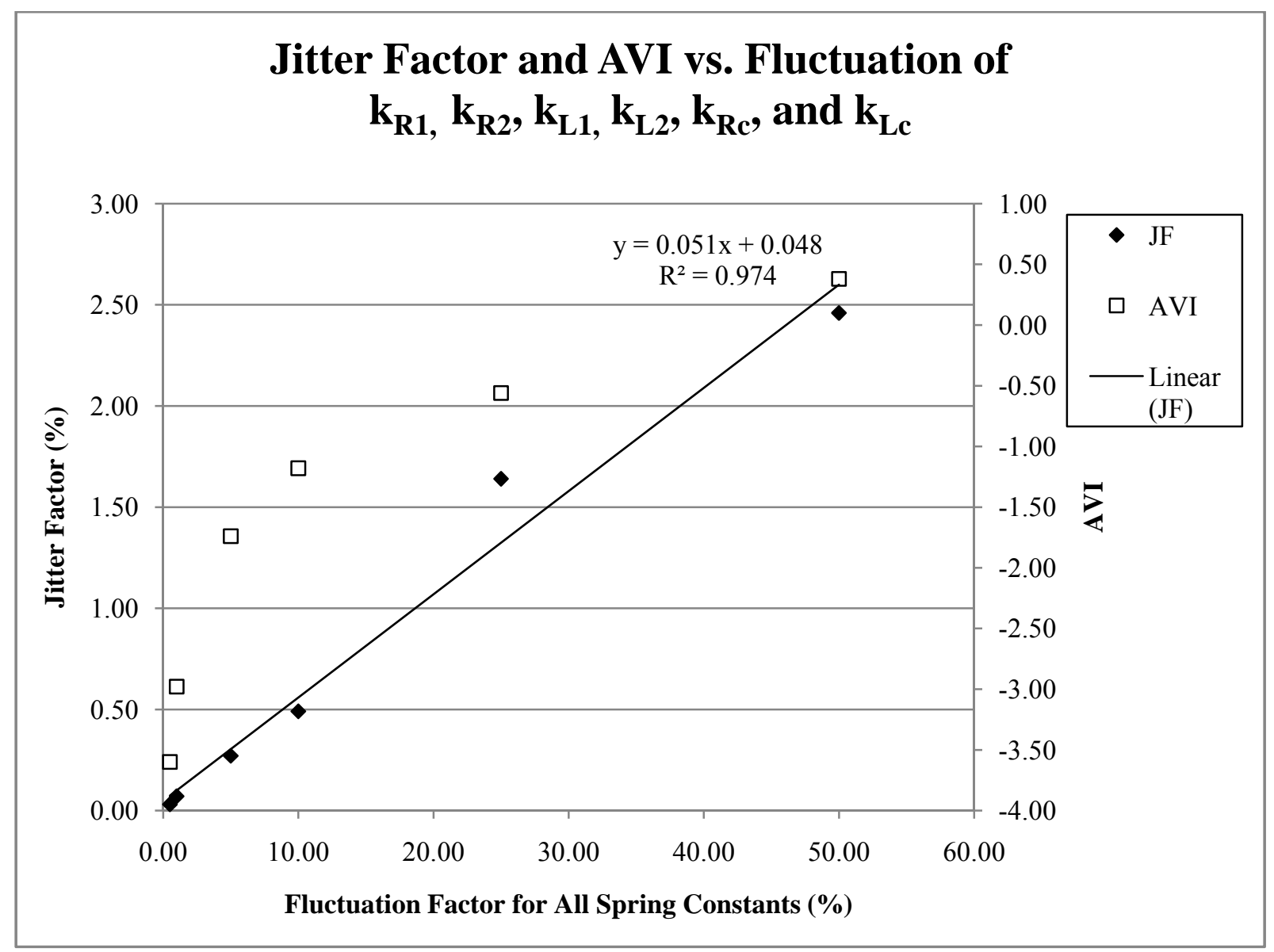

Figure 5-24 Jitter Factor and Amplitude Variability Index versus the magnitude of random fluctuation of all spring constants.

It was expected that random fluctuations of the spring constants would have caused more jitter and shimmer. The work by Titze (1991) stated that a root mean square variation of $1 \%$ in the tensile force of the muscles should result in approximately $0.5 \%$ jitter. Our study found that much larger fluctuations in the spring constants $(10 \%$ random fluctuations for a root mean square deviation of $5 \%$ ) was required to explain a JF of $0.5 \%$. One possibility for this discrepancy is that the fluctuations in the real muscle tension are more sporadic than the uniform random distribution applied here. Another possibility is that a lack of motor control may cause the 
pertinent muscle tensions to fluctuate to a much greater degree than that expected based on tetanic contraction measurements. On the other hand, one interpretation of our results is that the vocal folds, as represented by our asymmetric adaption of the IF72 model, may have inherent corrective mechanisms that smooth-out underlying fluctuations.

\subsection{Hypothesis \#3: Time-independent Asymmetry in the Driving Forces}

In this part of the study, the aerodynamic driving forces were modified by a constant multiplicative factor to determine whether or not these time-independent variations would result in jitter or shimmer. As discussed previously, the application of the M5 intraglottal pressure data requires that one vocal fold act as the flow-wall (FW) while the other acts as the non-flow wall (NFW). The M5 data indicates that the FW is subject to slightly higher negative pressures (inward forces) than the FW during the divergent part of the cycle. This pressure difference is due to the fact a greater portion of the fluid energy is dedicated to the kinetic energy of the air along the FW, leaving less potential energy to perform work on the FW vocal fold. Throughout this study, the right vocal fold was maintained as the NFW for all typical calculations. Based on a review of the graphical output for the typical case in Figure 5- 1, it appears that there are no detectable asymmetries in the glottal flow or the displacement of the oscillators. Closer examination indicates that the NFW oscillators $\left(\mathrm{m}_{\mathrm{R} 1}\right.$ and $\mathrm{m}_{\mathrm{R} 2}$ ) have slightly larger amplitudes than the FW ( $\mathrm{m}_{\mathrm{L} 1}$ and $\mathrm{m}_{\mathrm{L} 2}$ ), owing to the greater inward forces on the FW side. The jitter and shimmer plots of Figure 5-2 indicate that were no detectable perturbations in the fundamental frequency or amplitude of the glottal flow. Therefore, if one vocal fold is consistently selected as the FW, then jitter and shimmer are not observed even though the amplitudes of the oscillators are not symmetric. A comparison of the NFW and FW forces on the lower masses is presented in Figure 5-25. 


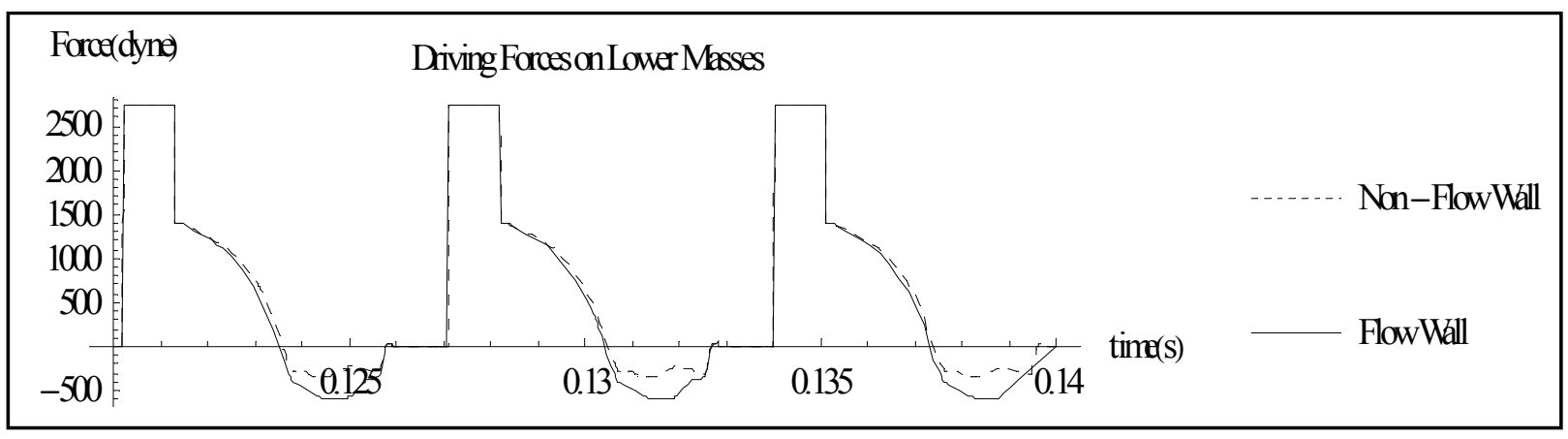

Figure 5-25 Driving forces on the lower masses during phonation.

To test this scenario further, the forces on the lower, right $\left(F_{R 1}\right)$ and the upper, right $\left(F_{R 2}\right)$ oscillators were systematically increased to investigate possible sources of jitter and shimmer. The forces were increased separately by factors ranging from 1.05 to 2.00 . The results indicate that no jitter or shimmer was detected in any case. However, the asymmetry in the motion of the right and left vocal folds is amplified with increasing left/right force asymmetry on the lower, right vocal fold. In contrast with the results of hypothesis \#1, in this case the right and left oscillators remained in phase with only a relative amplitude difference occurring. Once again, the oscillators entrained and found a new equilibrium position with a common frequency to stabilize the system. The oscillator displacement graphs for the case of increasing $F_{R 1}$ are provided in Figure 5-26. The increased force on the upper, right mass $\left(\mathrm{F}_{\mathrm{R} 2}\right)$ had no readily observable effect on the oscillator motion. Since the force $F_{R 1}$ is several times greater than $F_{R 2}$, any modifications to $F_{R 2}$ are overshadowed by $F_{R 1}$. Graphical output of the results for changes in $\mathrm{F}_{\mathrm{R} 2}$ reveal no new information and are therefore not provided. 


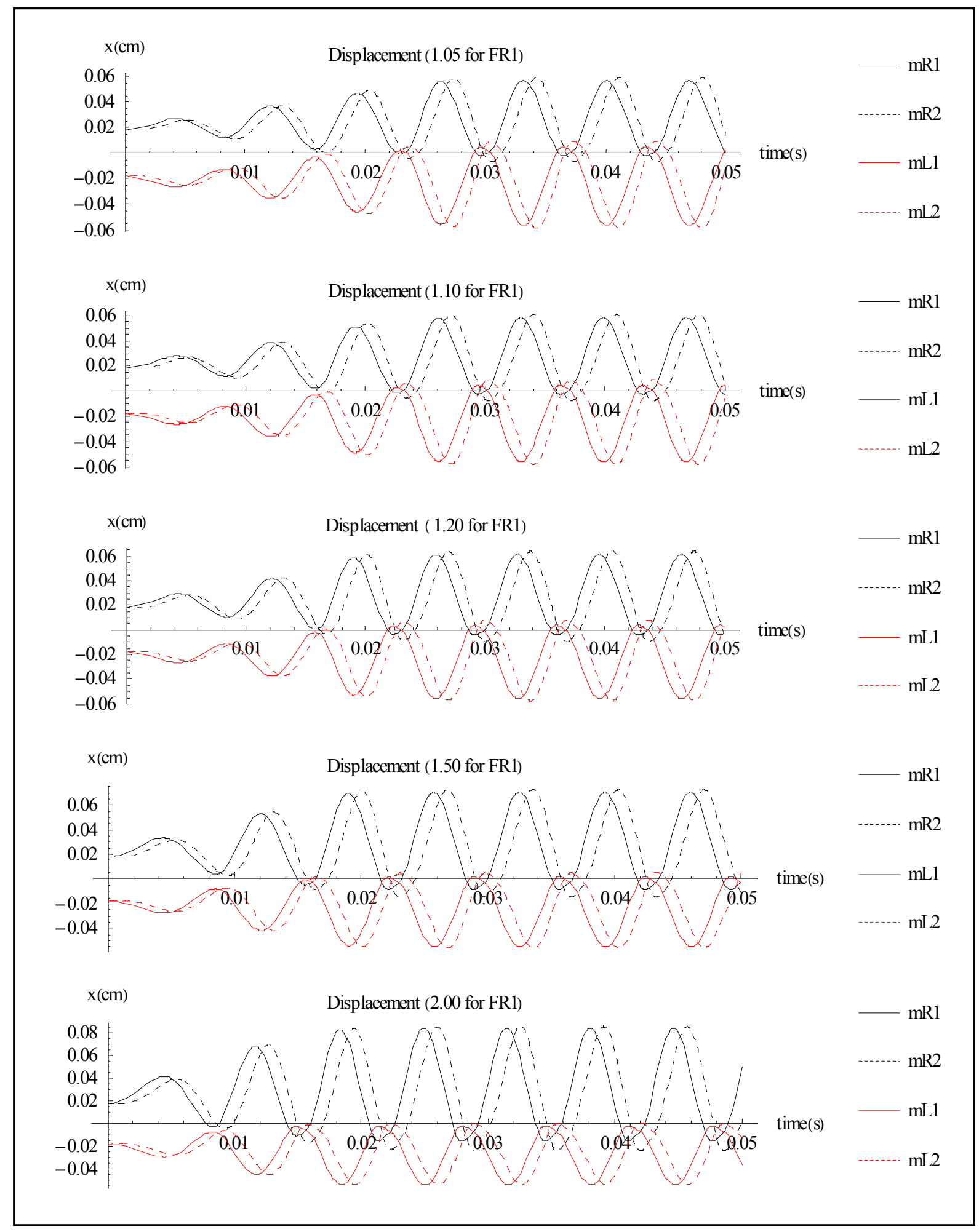

Figure 5-26 Oscillator displacement graphs for increased lower, right driving force $F_{R 1}$ by factors of $1.05,1.10,1.20,1.50$, and 2.00 (from top to bottom) 


\subsection{Hypothesis \#4: Time-dependent Asymmetry in the Driving Forces}

The driving forces were varied to simulate the effects of pressure imbalances in the glottis during phonation. As stated previously, an airflow jet forms due to the narrow constriction of the glottis and the high velocity of airflow. During the convergent part of the cycle the jet forms at a high point on the FW (somewhere along the medial surface of $\mathrm{m}_{\mathrm{L} 2}$ in our model). However, during the divergent part of the cycle, the jet forms at a lower point (upstream) on the FW (somewhere along the medial surface of $\mathrm{m}_{\mathrm{L} 1}$ ). The formation of the jet on the FW results in a stall of the airflow in the vicinity of the NFW which may lead to pressure imbalances within the glottis (Kline 1959). This imbalance leads to unpredictable flow patterns resulting in force fluctuations on the NWF. This effect was simulated by adding a random, timedependent perturbation to the forces on the NFW during the appropriate times of the cycle. First, only the force $F_{R 1}$ was perturbed by factors ranging from $+/-1 \%$ to $+/-50 \%$ during the divergent part of the cycle. Then, $F_{R 2}$ was perturbed during the entire cycle while $F_{R 1}$ was perturbed only during the divergent part of the cycle again. The fundamental period contour and glottal airflow amplitude contour for the most extreme perturbations (+/- 50\%) in both cases are presented in Figures 5-27 and 5-28.

As the contour plots indicate, the random fluctuations of the forces, even up to $50 \%$ variation, had little effect on variations in the period or amplitude of the glottal flow. This limited effect is likely due to the fact that the driving forces are perturbed at points in the cycle where the magnitudes of those forces are small. Inspection of Figure 5-25 shows that the inward forces on the lower portions of the vocal folds (represented here by $F_{R 1}$ ) are relatively weak. 

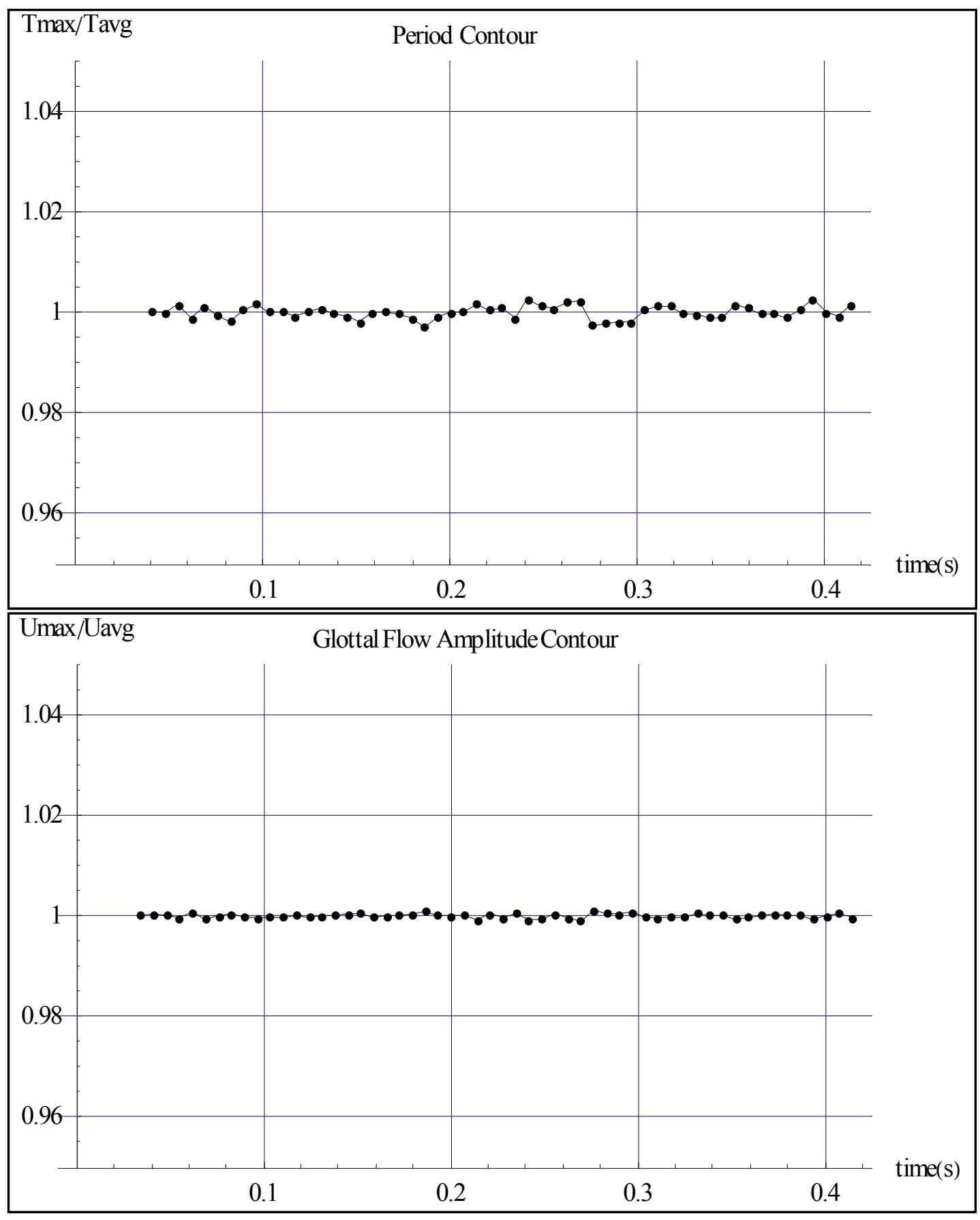

Figure 5-27 Fundamental period contour normalized to the average period and glottal airflow amplitude contour normalized to the average amplitude for $+/-50 \%$ random fluctuations of the driving force on the lower, right mass during the divergent part of the cycle. 

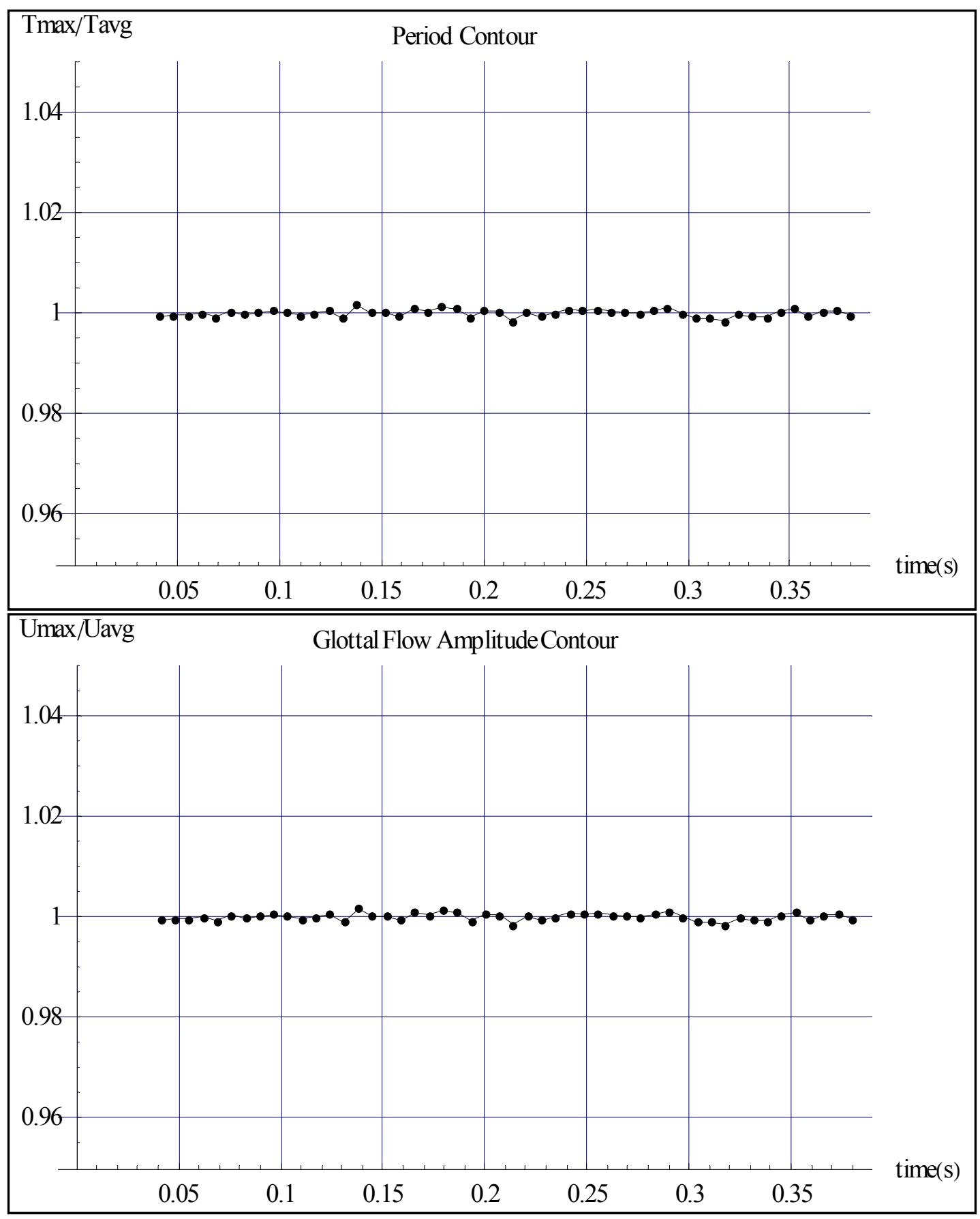

Figure 5-28 Fundamental period contour normalized to the average period and glottal airflow amplitude contour normalized to the average amplitude for $+/-50 \%$ random fluctuations of both driving forces on the right vocal fold. 
Also, the forces on the upper vocal folds (represented by $F_{R 2}$ ) are approximately ten times less that the forces on the lower portion. Because a perturbation of a weak force is still a weak force, these perturbations have little effect on the system. For the case of $F_{R 1}$ perturbation, numerical values for JF and AVI ranged from $0.003 \%$ to $0.12 \%$ and -6.31 and -3.70 , respectively. The linear correlation between jitter and shimmer was -0.09 , indicating that the period and amplitude perturbations are not linearly correlated. For the case of $F_{R 1}$ and $F_{R 2}$ both perturbed, numerical values for JF and AVI ranged from $0.002 \%$ to $0.07 \%$, and -6.27 and -4.04 , respectively. These values are much lower than those observed in normal voice. Again, there was insignificant linear correlation between jitter and shimmer, as indicated by the linear correlation coefficient of 0.09 . The JF and AVI values for the case of perturbed $F_{R 1}$ and $F_{R 2}$ are presented graphically in Figure 5-29.

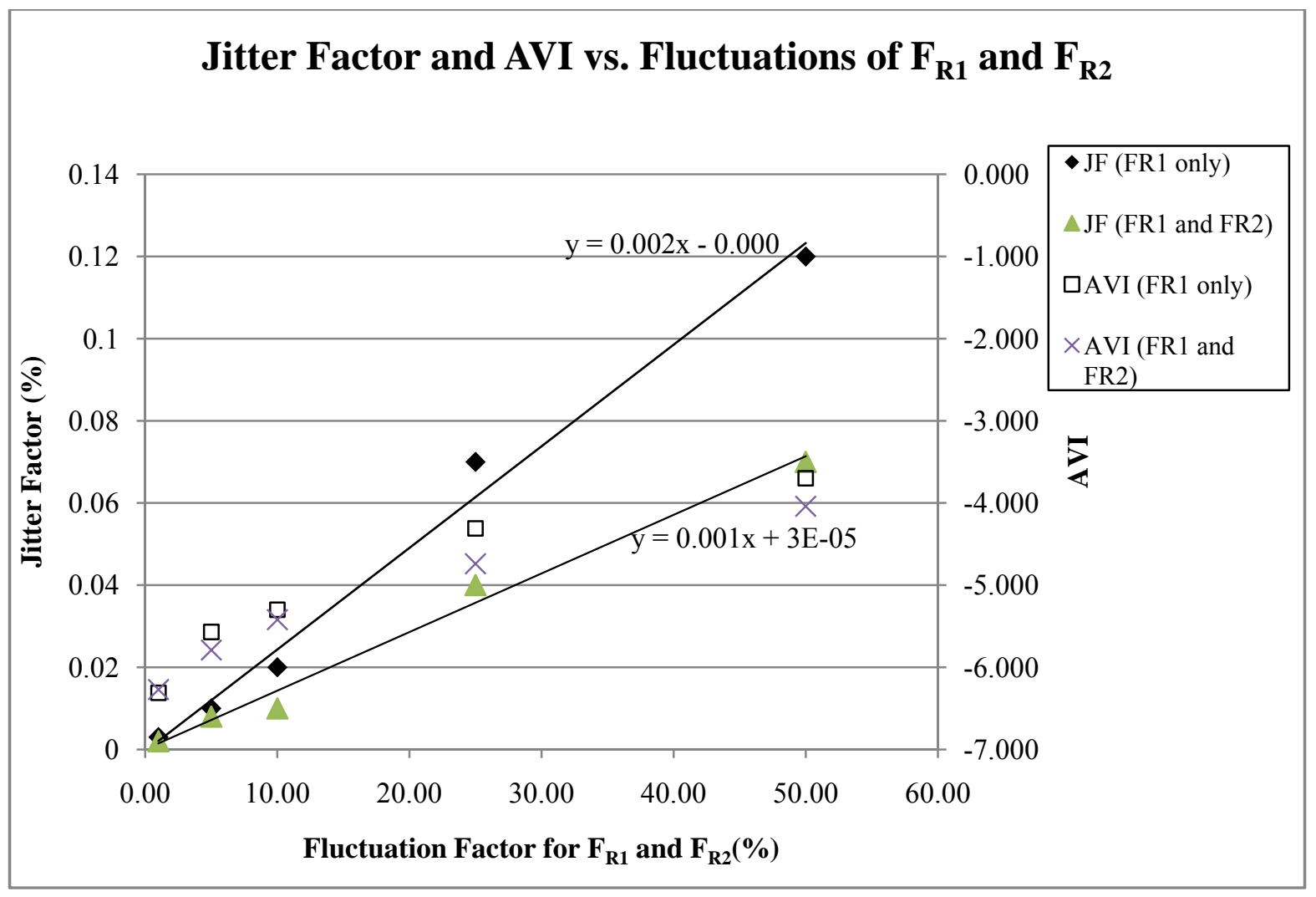

Figure 5-29 Jitter Factors and Amplitude Variability Index for random fluctuations of the driving forces. 
It may be postulated that the generation of vortices causes a dramatic increase in the kinetic energy of the fluid, thereby greatly reducing the potential energy (or static pressure) of the fluid. This would result in large, random suction forces during the divergent part of the cycle on the NFW. To test this possibility a final calculation was conducted with random fluctuations in the driving forces of as much as a factor of 2.00 greater than the steady-state value during the divergent part of the cycle. The results indicate that a JF of only $0.16 \%$ and minimal shimmer were detected for this case. The correlation coefficient between jitter and shimmer was 0.12 . The JF value of $0.16 \%$ represents approximately $32 \%$ of the expected value of $0.5 \%$ for normal voice. The fundamental period and glottal airflow amplitude contour plots are provided in Figure 5-30. 

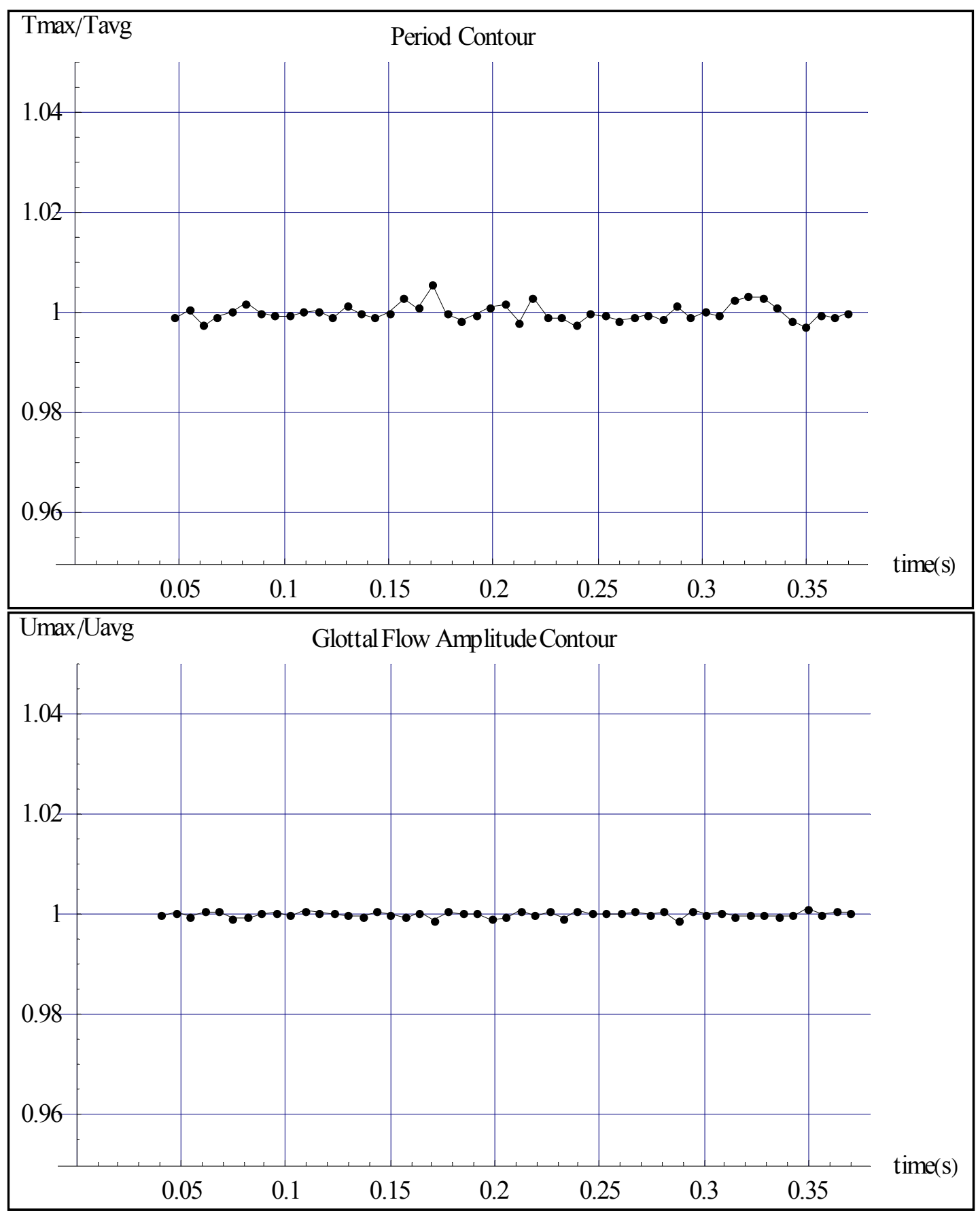

Figure 5-30 Fundamental period contour normalized to the average period and glottal airflow amplitude contour normalized to the average amplitude when the fluctuating forces on the right vocal fold are increase by as much as a factor of two. 


\section{CHAPTER 6. CONCLUSIONS}

This study set out to develop a mathematical model of the human voice with the requisite complexity to investigate possible causes of voice perturbations, namely, jitter and shimmer. Based on a review of the published literature, a lumped element, two-mass asymmetric model was developed using the well-known work by Ishizaka and Flanagan (1972) as a starting point. This renowned model was extended to incorporate bilateral asymmetry, similar to the subsequent work by Ishizaka and Isshiki (1976). These previous models exhibited unrealistically large inward forces during the closing part of the vocal fold cycle. The model employed in this study was unique because it remedied this deficiency by incorporating empirical data from a static model of the human larynx, known as the M5 model. The newly developed model was then employed to test four specific hypotheses as possible causes of jitter and shimmer for the case of an excised larynx. Numerical calculations and analysis were conducted using the software Mathematica to identify signatures of these voice perturbations.

Each hypothesis was tested by systematically varying specific biomechanical or aerodynamic parameters related to possible causes of jitter or shimmer. In each case, an attempt was made to find a connection between the modified parameter of the model and its physiological basis. This connection was often vague, but that is to be expected when attempting to model a physical system that has an almost infinite number of degrees of freedom using a model with only four. Nevertheless, the simplicity of the model, and the connection of the model parameters to at least some of the large scale features of the real system, makes it a useful analytical tool.

The following conclusions are based on the results obtained in this study:

1) No jitter or shimmer was detected for time-independent, asymmetric masses or tensions. 
2) No jitter or shimmer was detected for time-independent, asymmetric driving forces.

3) Randomly fluctuating asymmetric spring forces resulted in jitter and shimmer. The sizes of the jitter and shimmer perturbations were well below those expected in normal voice, even when the random fluctuations were extreme.

4) Randomly fluctuating driving forces also resulted in jitter and shimmer. The sizes of jitter and shimmer effects were less than those obtained for the asymmetric spring forces.

5) On average, the fluctuating spring forces caused more shimmer than jitter while the fluctuating driving forces caused more jitter than shimmer.

6) Fluctuating spring forces resulted in a significant, positive linear correlation between jitter and shimmer (from 0.25 to 0.45 ), while fluctuating driving forces did not result in a significant linear correlation between jitter and shimmer (from -0.1 to 0.12 ).

7) Although the time-independent physical (mass and tension) asymmetries did not cause jitter or shimmer, asymmetric motion was observed in the oscillators. Specifically, the right and left vocal folds were out of phase, and differed in amplitude, but shared the same frequency. The fundamental frequency varies more rapidly with the mass parameters than with the tension parameters.

8) Asymmetric vocal fold motion was also observed for the cases of time-independent asymmetric driving forces. For these cases, however, the vocal folds remained in phase but the amplitudes of the left and right sides were different. Once again, the vocal folds vibrated at a common frequency.

In summary, jitter and shimmer result from time-dependent asymmetries. Some physical quantity must change from cycle to cycle for a perturbation in the output sound to be detected. None of the time-independent modifications resulted in measurable jitter or shimmer. Rather, a 
surprising observation was made that the dynamics of the model required that the vocal folds entrain, retaining the overall periodicity of the glottal flow. In these cases, an asymmetry was apparent in the amplitudes or phases of the oscillators, but the asymmetry did not express itself in the time-dependence, since the asymmetric motion repeated itself exactly during each cycle.

The observation of hidden asymmetries raises the question; do time-independent physical asymmetries cause time-dependent effects that are not detectable by this model? If the model forces were more elaborate and depended on the angle of each vocal fold separately, then a cycle-to-cycle perturbation in the forces might occur because the relative angles may be different from cycle to cycle. On the other hand, the results presented here may indicate that asymmetric driving forces have less of an effect on vocal fold motion than one might expect. It appears that the coupling of the vocal folds across the glottal area often leads to a robust entrainment, which tends to mask asymmetries in the vocal fold motions. That is, the changes to the forces on one side affect the other, rather than forces acting in isolation. This coupling can only be observed in a dynamic model because it is the motion of the vocal folds that facilitates the connection between them.

Further work pertaining to this study should entail expanding the force function employed here by including additional empirical data. The model could be greatly improved by the addition of asymmetric force data, as well as the inclusion of the interaction between the sound source and the vocal tract. Also, similar models with more degrees of freedom may exhibit unique asymmetries that could result in jitter or shimmer. Regardless of the model, future studies should also include the effects of varying the subglottal pressure and prephonatory area. 
Although the exact mechanisms of vocal fold asymmetry are unknown, the type of model employed herein can be used to investigate a wide range of possible scenarios, with significant implications for the simulation of natural sounding voice, and the understanding and treatment of voice pathologies. 


\section{REFERENCES}

Alipour-Haghighi, F. and I. R. Titze (1983). Simulation of particle trajectories of vocal fold tissue during phonation. Vocal Fold Physiology: Biomechanics, Acoustics, and Phonatory Control I. R. Titze and R. C. Scherer. Denver Center for the Performing Arts, Denver, Co.: 183-190.

Alipour-Haghighi, F. and I. R. Titze (1989). "Tetanic Contraction in Vocal Fold Muscle." Journal of Speech \& Hearing Research 32: 226-231.

Alipour-Haghighi, F., I. R. Titze and P. Durham (1987). "Twitch Response in the Canine Vocalis." Journal of Speech \& Hearing Research 30: 290-294.

Baer, T. (1975). Investigation of Phonation Using Excised Larynxes. Cambridge, MA. MIT. Ph.D Dissertation

Baer, T. (1978). "Effect of single-motor-unit firings on fundamental frequency of phonation." Journal of the Acoustical Society of America 64(S90(A)).

Baer, T. (1980). Vocal jitter: A neuromuscular explanation. Eighth Symposium: Care of the Professional Voice, New York, NY, The Voice Foundation.

Baken, R. J. and R. F. Orlikoff (2000). Clinical Measurement of Speech and Voice. San Diego, CA, Singular Publishing Group.

Deal, R. E. and F. W. Emanuel (1978). "Some waveforms and spectral features of vowel roughness." Journal of Speech \& Hearing Research 21: 250-264.

Doellinger, M., D. Berry and D. Montegquin (2004). "Vocal tract influence on medial surface dynamics of the vocal folds (A)." Journal of the Acoustical Society of America 116(4): $2545-2546$.

Fant, G. (1960). Acoustic Theory of Speech Production. The Hague, Mouton \& Co. N.V. 
Farnsworth, D. W. (1940). "High-Speed Motion Pictures of the Human Voice "Bell Labs(7): 203-208.

Flanagan, J. L. (1972). Speech Analysis, Synthesis and Perception. New York, Berlin, SpringerVerlag.

Flanagan, J. L. and L. Landgraf(1968). "Self-oscillating source for vocal tract synthesizers." IEEE Trans. Audio Electroacoust. AU-16: 57-64.

Fulcher, L., R. C. Scherer, A. Melnykov, V. Gateva and M. E. Limes (2006). "Negative coulomb damping, limit cycles and self-oscillation of the vocal folds." American Journal of Physics 74(5): 386-393.

Hanavan, P. C. (2006). Anatomy of Phonation, from http://faculty.augie.edu/ p pchanavan/anatomy/AP_Chapter5.ppt.

Hirano, M. (1974). "Morphological structure of the vocal cord as a vibrator and its variations." Folia Phoniatr. 26: 89-94.

Hirano, M. (1975). "Phonosurgery: Basic and Clinical Investigations." Otologia (Fukuoka) 21: 239-240.

Hirano, M., S. Hibi, S. Yoshida, Y. Hirade, H. Kasuya and Y. Kikuchi (1988). "Acoustic analysis of pathological voice." Acta Otolaryngologica 105: 432-438.

Hollien, H., J. Michel and E. T. Doherty (1973). "A method for analyzing vocal jitter in sustained phonation." Journal of Phonetics 1: 85-91.

Ishizaka, K. and J. L. Flanagan (1972). "Synthesis of Voiced Sounds From a Two-Mass Model of the Vocal Cords." The Bell System Technical Journal 51(6): 1233-1267.

Ishizaka, K. and N. Isshiki (1976). "Computer simulation of pathological vocal-cord vibration." Journal of the Acoustical Society of America 60(5): 1193-1198. 
Ishizaka, K. and T. Kaneko (1968). "On the Equivalent Mechanical Constants of the Vocal Folds." Journal of the Acoustical Society of Japan 24(5): 312-313.

Jiang, J. J., I. R. Titze, D. B. Wexler and S. D. Gray (1994). "Fundamental frequency and amplitude perturbation in reconstructed canine vocal folds." Annals of Otology, Rhinology, and Laryngology 103: 145-148.

Jiang, J. J., Y. Zhang and C. McGilligan (2006). "Chaos in Voice, from Modeling to Measurement." Journal of Voice 20(1): 2-17.

Kelly, J. and C. Lochbaum (1962). Speech Synthesis. Prac. Fourth International Congress on Acoustics.

Kline, S. J. (1959). "On the Nature of Stall." Journal of Basic Engineering 81(Series D): 305322.

Liljencrants, J. (1985). Speech Synthesis with a Reflection-Type Line Analog. Stockholm, Sweden. Royal Inst. of Tech. DS Dissertation

Lowell, S. Y. and B. H. Story (2006). "Simulated effects of the cricothyroid and thyroarytenoid muscle activation on adult-male vocal fold vibration." Journal of the Acoustical Society of America 120(1): 386-397.

Milenkovic, P. (1987). "Acoustic tube reconstruction from noncausal excitation." IEEE Trans. Acous. Speech and Sig. Proc. ASSP-35(8): 1089-1100.

Neubauer, Z. Zhang, R. Miraghaie and D. Berry (2007). "Coherent structures of the near field flow in a self-oscillating physical model of the vocal folds " Journal of the Acoustical Society of America 121(2): 1102-1120.

Orlikoff, R. F. and R. J. Baken (1989a). "The Effect of the Heartbeat on Vocal Fundamental Frequency Perturbation." Journal of Speech \& Hearing Research 32: 576-582. 
Orlikoff, R. F. and R. J. Baken (1989b). "Fundamental frequency modulation of the human voice by the heartbeat: Preliminary results and possible mechanisms " Journal of the Acoustical Society of America 85(2): 888-893.

Pelorson, X., A. Hirschberg, R. R. van Hassel, A. P. J. Wijnands and Y. Auregan (1994). "Theoretical and experimental study of quasisteady-flow separation within the glottis during phonation. Application to a modified two-mass model." Journal of the Acoustical Society of America 96(6): 3416-3431.

Rahn, D. A. I., M. Chou, J. J. Jiang and Y. Zhang (2007). "Phonatory Impairment in Parkison's Disease: Evidence from Nonlinear Dynamic Analysis and Perturbation Analysis." Journal of Voice 21(1): 64-71.

Scherer, R. C. M5 experimental data of pressure distributions in the vocal folds (unpublished). Bowling Green State University, Dept. of Communication Disorders.

Scherer, R. C., D. Shinwari, K. J. De Witt, C. Zhang, B. R. Kucinschi and A. A. Afjeh (2001). "Intraglottal pressure profiles for a symmetric and oblique glottis with a divergence angle of 10 degrees." Journal of the Acoustical Society of America 109(4): 1616-1630.

Scherer, R. C., D. Shinwari, K. J. De Witt, C. Zhang, B. R. Kucinschi and A. A. Afjeh (2002). "Intraglottal pressure distributions for a symmetric and oblique glottis with a uniform duct (L)." Journal of the Acoustical Society of America 112(4): 1253-1256.

Scherer, R. C. and I. R. Titze (1983). Pressure Flow Relationships in a Model of the Laryngeal Airway with a Diverging Glottis. Vocal Fold Physiology: Contemporary Research and Clinical Issues. D. Bless and J. Abbs. San Diego, College Hill: 179-193.

Steinecke, I. and H. Herzel (1995). "Bifurcations in an asymmetric vocal-fold model." Journal of the Acoustical Society of America 97(3): 1874-1884. 
Story, B. H. (1995). Physiologically-Based Speech Simulation Using an Enhanced WaveReflection Model of the Vocal Tract. Iowa City, Iowa. University of Iowa. Ph.D. Dissertation

Story, B. H. and I. R. Titze (1995). "Voice simulation with a body-cover model of the vocal folds." Journal of the Acoustical Society of America 97(2): 1249-1260.

Thapa, P. (2005). Pressure Distributions in a Static Hemilarynx Model. Bowling Green, Ohio. Bowling Green State University. Master of Science Thesis

Titze, I. R. (1973). "The Human Vocal Cords: A Mathematical Model Part I." Phonetica 28(3-4): 129-170.

Titze, I. R. (1974). "The Human Vocal Cords: A Mathematical Model Part II." Phonetica 29(12): 1-21.

Titze, I. R. (1984). "Parameterization of the glottal area, glottal flow, and vocal fold contact area." Journal of the Acoustical Society of America 75(2): 570-580.

Titze, I. R. (1991). "A Model for Neurologic Sources of Aperiodicity in Vocal Fold Vibration." Journal of Speech \& Hearing Research 34: 460-472.

Titze, I. R., S. S. Schmidt and M. R. Titze (1995). "Phonation threshold pressure in a physical model of the vocal fold mucosa " Journal of the Acoustical Society of America 97(5): 3080-3084.

Titze, I. R. and D. T. Talkin (1979). "A theoretical study of the effects of various laryngeal configurations on the acoustics of phonation." Journal of the Acoustical Society of America 66(1): 60-74.

Tokuda, I. and H. Herzel (2005). "Detecting synchronizations in an asymmetric vocal fold model from time series data." Chaos 15(1): 013702-01 - 013702-11. 
van den Berg, J. W., J. T. Zantema and J. Doornenbal, P. (1957). "On the Air Resistance and Bernoulli Effect of the Human Larynx." Journal of the Acoustical Society of America 29: 626-631.

Voice Medicine (2006). "Normal Voice Function." from http://www.voicemedicine.com/normal_voice functioning.htm.

Zhang, Y., C. McGilligan, L. Zhou, M. Vig and J. J. Jiang (2004). "Nonlinear dynamic analysis of voices before and after surgical excision of vocal polyps." Journal of the Acoustical Society of America 115(5): 2270-2277. 